Running Head: GENERAL RESPONSE PROCESS THEORY FOR SJTS

\title{
A General Response Process Theory for Situational Judgment Tests
}

\author{
James A. Grand \\ University of Maryland
}

\section{Citation:}

Grand, J.A. (in press). A general response process theory for situational judgment tests. Journal of Applied Psychology.

\section{Author's Note:}

I am indebted to Mike Braun, Goran Kuljanin, Jennifer Wessel, Paul Hanges, and Mike Dougherty for their review and helpful comments on early drafts of the manuscript, computational model, and web application, as well as Ben Levine for his assistance collecting the empirical data reported in the paper. Additionally, I would like to acknowledge the University of Maryland supercomputing resources (https://oacs.umd.edu/oacs-cloud/bsos-high-performancecomputing-cluster) made available for conducting the research reported in this paper. Portions of this work were previously presented at the 2017 and 2019 annual meetings of the Society for Industrial and Organizational Psychology.

Contact Information:

James A. Grand, Department of Psychology, 3147A Biology-Psychology Building, University of Maryland, College Park, MD 20742, phone: (301) 405-5935, email: grandjam@umd.edu

(C) 2019, American Psychological Association. This paper is not the copy of record and may not exactly replicate the final, authoritative version of the article. Please do not copy or cite without authors' permission. The final article will be available, upon publication, via its DOI: 10.1037/apl0000468 


\begin{abstract}
Situational judgment tests (SJTs) have emerged as a staple of assessment methodologies for organizational practitioners and researchers. Despite their prevalence, many questions regarding how to interpret respondent choices or how variations in item construction and instruction influence the nature of observed responses remain. Existing conceptual and empirical efforts to explore these questions have largely been rooted in reflexive psychometric measurement models that describe participant responses as indicative of (usually multiple) latent constructs. However, some have suggested that a key to better understanding SJT responses lies in unpacking the judgment and decision-making processes employed by respondents and the psychological and contextual factors that shape how those processes play out. To this end, the present paper advances an integrative and generalizable process-oriented theory of SJT responding. The framework, labeled Situated Reasoning and Judgment (SiRJ), proposes that respondents engage in a series of conditional reasoning, similarity, and preference accumulation judgments when deciding how to evaluate and respond to an SJT item. To evaluate the theory's plausibility and utility, the SiRJ framework is translated into a formal computational model and results from a simulation study are analyzed using neural network and Bayesian survival analytic techniques that demonstrate its capability to replicate existing and new empirical effects, suggest insights into SJT interpretation and development, and stimulate new directions for future research. An interactive web application that allows users to explore the computational model developed for SiRJ (https://grandjam.shinyapps.io/sirj) as well as all reported data and the full model/simulation code (https://osf.io/uwdfm/) are also provided.
\end{abstract}

Keywords: situational judgment tests; judgment; decision-making; computational modeling 
From political leaders to CEOs to front-line employees, there is widespread recognition that the capacity to make effective decisions and accurate judgments is a sign of competence and expertise. This recognition is a key motivation for the use and popularity of low-fidelity simulations in personnel selection and assessment (Weekley, Hawkes, Guenole, \& Ployhart, 2015). Among the most familiar low-fidelity simulation methods are situational judgment tests (SJTs), in which individuals are presented with a series of scenarios that reflect possible circumstances, tasks, or decisions that might be experienced in a particular context and asked to identify how to address these demands (Motowidlo, Dunnette, \& Carter, 1990; Motowidlo \& Tippins, 1993). Respondents' answers to SJT items are often then correlated with normative proficiency standards (e.g., choices identified as effective, desirable, or appropriate by domain experts) or other variables of interest (e.g., job performance, cognitive ability, personality, interests, etc.) to generate a performance score or, in some cases, infer construct validity. In personnel management contexts, SJT data may also be used to draw conclusions about future decisions or behaviors an individual might demonstrate on the job. A large body of research now exists demonstrating that SJTs show favorable levels of face validity, criterion validity, and are capable of improving predictions of job performance above and beyond ability and dispositional measures (Christian, Edwards, \& Bradley, 2010; McDaniel, Morgeson, Finnegan, Campion, \& Braverman, 2001; Weekley et al., 2015).

Despite converging evidence of their practical and predictive value, many fundamental questions related to the use and design of SJTs persist. Perhaps most significantly, there is still a great deal of uncertainty regarding how to interpret SJT performance scores. A survey of the research literature on this topic reveals three common perspectives: (1) SJT scores reflect multiple constructs that can be independently measured and scored (e.g., Chan \& Schmitt, 2005; Oswald, Schmitt, Kim, Ramsay, \& Gillespie, 2004; Sorrel, Olea, Abad, de la Torre, Aguado, \& Lievens, 2016); (2) SJT scores reflect higher-order composite constructs that cannot be readily decomposed (e.g., interpersonal skills, teamwork skills; Lievens, Buyse, \& Sackett, 2005; Lievens \& Sackett, 2007; Mumford, van Iddekinge, Morgeson, \& Campion, 2008); and (3) SJT scores reflect indeterminate concepts such as "common sense," everyday reasoning, practical intelligence, or general domain knowledge (e.g., Lievens \& Motowidlo, 2016; McDaniel \& Nguyen, 2001; Sternberg, Wagner, Williams, \& Horvath, 1995). However, comparing the veracity of these conceptual positions has proven challenging, if not intractable. The multidimensional nature of SJT scores means they often share moderately strong correlations with a wide variety of dispositions, abilities, experience, and knowledge that are themselves correlated (McDaniel et al., 2001; McDaniel \& Nguyen, 2001). Isolating a coherent 
and consistent mapping between latent psychological constructs and performance on an SJT—or even a single SJT item—thus remains an elusive target.

In addition to the ambiguities associated with interpreting SJT performance, similar uncertainty exists regarding how the construction and presentation of an SJT item influences respondents' choices and subsequent interpretation of scores. For example, Lievens and Sackett (2007) note that there is no theory or empirical guidance to explain what makes some SJT items function more poorly or appear more "difficult" than others. Explanations for how or why differences in instructions (i.e., select what one "would" versus "should" do, select most versus least effective responses; McDaniel \& Nguyen, 2001; Ployhart \& Erhart, 2003) or item design (i.e., soliciting open-ended versus multiple-choice responses, using item stems/situations with high versus low specificity; Krumm et al., 2015; Rockstuhl, Ang, Ng, Lievens, \& Van Dyne, 2015) influence SJT responding and their implications for interpreting respondent performance are also poorly understood and under-researched.

There is increasing recognition that efforts to better interpret the nature of SJTs will likely require greater consideration of the underlying psychological processes employed by individuals when evaluating and responding to SJT items (e.g., Brooks \& Highhouse, 2006; Chan \& Schmitt, 2005; Foldes et al., 2010; Leeds, 2012; Ployhart, 2006; Stemler \& Sternberg, 2006). Though their specific content, development, and implementation are often varied to suit the needs of assessment, SJTs are inherently tools that reflect the judgment and decision-making of respondents-a fact even explicitly acknowledged by name in the case of SJTs. Judgment can be generally defined as the "decisionmaking process concerned with assessing, estimating, and inferring what events will occur and what the decisionmaker's evaluative reactions to those outcomes will be" (Hastie, 2001, p. 657). Although this description appears to capture the manner by which respondents answer SJT items, surprisingly little attention has been directed towards examining actual judgment and decision-making during SJT responding and their implications for SJT measurement, design, and interpretation. This irony is further puzzling when one considers that some SJT applications may be explicitly interested in the judgment quality of respondents rather than (or in addition to) their standing on particular psychological constructs.

To illustrate the value of understanding the cognitive processes underlying SJT responding, consider the following item that might be included on an SJT for law enforcement officers (Brooks and Highhouse, 2006, p. 39):

You are shopping when you notice a man robbing the store. What would you do?

A. Leave the store as quickly as possible and call the police.

B. Try to apprehend the man yourself.

C. Follow the man and call the police as soon as he appears settled somewhere.

D. Do nothing as you do not wish to get involved in the matter. 
Such an item is typical of an SJT and is comprised of three elements: an item stem that presents a situation/set of circumstances (e.g., "You are shopping when you notice a man robbing the store"); a set of response options from which the respondent may choose (e.g., "Leave the store as quickly as possible and call the police," "Try to apprehend the man yourself'); and an instructional prompt that indicates how the respondent is to evaluate the response options in relation to the item stem (e.g., "What would you do?"). The modal approach to interpreting respondents' choices for this question involve examining the correlations between selection of a response option and trait-level dispositional measures, expert opinion, or incumbent job performance ratings and using those criterion relations to ascribe characteristics/attributes to the participant (e.g., Lievens, Peeters, \& Schollaert, 2008; Motowidlo \& Beier, 2010; Motowidlo, Hooper, \& Jackson, 2006b; Weekley \& Jones, 1999). However, these procedures tell us little about how or why a respondent came to prefer one response over another, what considerations entered into this determination, or whether and/or why a person may have difficulty selecting a response to this situation. Furthermore, it would be challenging to use such data to anticipate the consequences for respondent behavior if the instruction set were changed to ask participants what they would not do or if more/different details are added to the situation (e.g., the robber is armed with a weapon, your family is in the store shopping with you). To begin addressing such questions necessitates elaboration of the judgment and decision-making processes respondents employ when they formulate a response to SJT items. Although it happens quickly, answering an SJT item is a complex cognitive operation in which individuals evaluate and identify the characteristics of the situation, assess potential responses to predict what might happen in each circumstance, and differentiate among the utility of different actions.

Advancing a general theory of SJT responding that describes how and why different individuals may reach different conclusions, what those responses might say about their future reasoning, and how to interpret the meaning of selected responses would be beneficial for interpreting the nature of SJT performance. It could also hold important implications for both SJT researchers and practitioners (Foldes et al., 2010; Ployhart, 2006). For researchers, understanding how respondents evaluate and provide responses to SJT items is important for elucidating the role of individual, contextual, and design factors on SJT performance (e.g., why might applicants score differently than incumbents, what are the conceptual implications for relying on different SJT scoring methods) as well as when, why, and to what extent SJT responses would be expected to differentially relate to other criteria of interest (e.g., dispositions, knowledge, experience). Practitioners could similarly benefit from this knowledge by being able to make more informed choices about the design and development of SJTs (e.g., which/why should certain SJT items be included in a battery, how should situations be presented to and responses solicited from respondents). Lastly, better 
understanding how to decipher SJT responding would enhance the transparency of SJT measurement and its relevance as a tool for making decisions. Citing Guion's reflections (1998, p. 618) on personnel assessment techniques, Brooks and Highhouse (2006) remind us that although we can make valid decisions without knowing what we are measuring, "at this point in the history of employment psychology, we should be getting tired of not knowing what we are doing, no matter how carefully we do it."

The purpose and motivation of the present paper is to begin addressing this gap by advancing a general theory and response process model for describing how individuals make choices on SJTs. Following review of existing work on judgment and decision-making in SJTs, the proposed response process theory-labeled Situated Reasoning and Judgment (SiRJ)—and its application to SJT responding is presented. The SiRJ framework integrates and builds upon existing work in the cognitive sciences to propose a model of the psychological processes and cognitive mechanisms involved in SJT responding. Next, the translation of SiRJ into a formal computational model is described. Results from a simulation study with the model are then presented to evaluate the sufficiency and plausibility of the theory as a representation of SJT responding. This is accomplished by contrasting data generated by the model against effects observed in empirical data with real SJT respondents. The paper concludes with a discussion of the utility of SiRJ for addressing fundamental questions about SJT performance and responding, and its potential to guide future research examining the nature of judgment and decision-making in SJTs.

\section{Judgment and Decision-making in SJTs}

A variety of frameworks attempting to describe the meaning and interpretation of SJT performance have been proposed. Arguably the most common framework conceptualizes an SJT as a method for measuring one or more latent constructs by examining (usually post hoc) how the endorsement of particular responses to SJT items correlate with a person's standing on one or more trait-level measures of those constructs. For example, Sorrel et al. (2016) describe the use of statistical cognitive diagnosis models for interpreting SJT responding. This analytical approach assumes that the probability of an individual choosing the most effective response to an SJT item is increased for individuals who possess a particular attribute or set of attributes and that the constellation of attributes needed to select the most effective response to any given item varies across situations/items. Cognitive diagnosis models thus treat SJTs as multidimensional measures and provide a means for simultaneously estimating respondents' standing on multiple latent constructs. Motowidlo and colleagues (Motowidlo et al., 2006a; 2006b) program of SJT research on implicit trait policies largely adheres to a similar logic. Drawing from research on personality and attitude expression, these authors propose that (a) people possess implicit beliefs about the relations 
among dispositional traits and behavioral effectiveness and (b) those who possess more of a particular trait are attracted to and therefore more likely to endorse behavioral responses which express that trait (e.g., "I believe being agreeable is generally good; thus behaviors that express more agreeableness are better than those that express less agreeableness"). Under this model, a person's response to an SJT item reflects individual differences in personality and beliefs in the effectiveness of expressing a given personality trait across situations. Subsequent work in this stream of research has also sought to differentiate the role of these implicit beliefs from specific job knowledge that may inform the utility of particular behavioral expressions presented as part of an SJT item (Lievens \& Motowidlo, 2016; Motowidlo \& Beier, 2010).

Although the cognitive diagnostic and implicit trait models of SJT performance acknowledge that judgment and decision-making play a role in respondent choices, they do not explicate what these processes are or how they influence SJT responding. This is not a fault of these (and other reflexive/construct-based) models per se, but rather a consequence of their conceptual foundation as psychometric rather than psychological models of SJT responding. In other words, these frameworks seek to describe the likelihood a respondent possesses more or less of a construct given the (pattern of) manifest response options they endorse. However, the link between cognitive processing and behavior (i.e., SJT response selection) remains unspecified. The lack of an underlying psychological theory for how respondents answer SJT items makes it difficult-if not impossible—-to address the lingering questions regarding SJT interpretation and design raised previously (e.g., what makes an SJT item more "difficult," how to interpret differences in SJT performance when respondents select the most versus least effective response, the effect of providing more versus less detail in the situations or responses of SJT items).

Attempts to more directly describe the judgment and decision-making processes involved in SJT responding exist, though they have tended to provide more abstracted accounts. For example, Foldes et al. (2010) present a conceptual review of how the various judgment and decision paradigms from the broader decision sciences literature (e.g., heuristics and biases, dual process theories, fast and frugal heuristics, recognition-primed decision-making) could be applied to understanding SJT performance. Stemler and Sternberg (2006) proposed that SJT responding reflects "practical intelligence," which they define as an individual's capability to successfully employ learned condition-action pairings (i.e., IF <situation $X>$, THEN $<$ do action $Y>$, BECAUSE <rationale $Z>$ ) to meet the needs of a given context. These authors suggest that respondents process SJT items by reconciling interpretations of the scenario/situation presented to them, identifying and selecting goal-directed response strategies (i.e., conditionaction pairings) to address the perceived demands of the situation, and that this processing is influenced by an 
individual's experiences, dispositions, and the sociocultural context. As a final example, Brooks and Highhouse (2006) posited that SJT responding taps into "quasi-rationality," or the application of intuitive (judgment characterized by an indeterminate, difficult to express, and automatic reasoning process) and analytical reasoning (judgment characterized by a logical, transparent, and controlled reasoning process) to make SJT response selections.

Although existing conceptualizations suggest intriguing linkages between SJT responding and potential judgment and decision-making mechanisms, they fall short of articulating a theoretical account of the cognitive response processes underlying SJT performance (Chan \& Schmitt, 2005). However, there are two noteworthy exceptions. The first is Ployhart's (2006) predictor response process model, which proposes that respondents engage in four cognitive processing stages when answering SJT items: comprehension (interpreting situation), retrieval (retrieving relevant information from memory), judgment (integrating retrieved information to form assessment of situation), and response selection (editing judgments to fit provided response options). Ployhart (2006) described how this conceptual framework might be integrated with latent construct modeling techniques to examine whether differences in individuals (e.g., test taking motivation, reading comprehension, experience, etc.) or items (e.g., instructions, situation specificity, etc.) influence one or more of these stages in ways that manifest as differences in SJT response selection. A second theoretical account of SJT responding is Leeds' (2012) theory of cognitive acuity, which frames SJT performance as differences in an individual's capacity to detect "correctness" among situation-specific responses. Drawing from psychophysical and signal detection research, the theory conceives of SJT response options as possessing effectiveness "signals" that convey the appropriateness of a response behavior in the given scenario. Leeds (2012) posited that SJT respondents attempt to detect these signals in the response options for an SJT item and then contrast them by evaluating all possible response option pairs to determine which behavior possesses the "strongest" effectiveness signal (e.g., Is "Leave the store as quickly as possible and call the police" more correct than "Try to apprehend the man yourself"?; Is "Try to apprehend the man yourself' more correct than "Do nothing as you do not wish to get involved in the matter"?). The option winning the most such competitions is subsequently endorsed as most effective.

The models proposed by Ployhart (2006) and Leeds (2012) represent important advancements towards addressing the cognitive process mechanisms involved in SJT responding. However, they also possess certain limitations. The most significant challenge for the predictor response process model is deriving and evaluating predictions to evaluate its core propositions-a requisite condition for any theory. Notably, the model is predicated on the ability for researchers to uniquely and accurately quantify dynamic cognitive processes as static latent 
constructs/outcomes on which individuals might vary. For instance, Ployhart (2006) posits that respondents' personality characteristics are likely to influence comprehension of the situation presented in an SJT item stem when instructed to select how they "would" respond in a given circumstance, but not when asked to indicate what they "should" do. To test this proposition though, a researcher would need to (a) define, quantify, and justify what it means to "comprehend" a situation (e.g., comprehend it "better?" Differently? More quickly?); (b) accurately distinguish when participants are engaged in comprehension versus a different unobservable cognitive process; and (c) reliably measure these concepts. Even if one could surmount these empirical obstacles, the model also does not offer a clear rationale as to why or in what way respondent characteristics should influence the comprehension of situations presented in an SJT item stem. Consequently, though the predictor response process model is intuitively appealing and offers a compelling conceptual linkage between psychometric and cognitive processing models, it is challenging to envision how its core mechanisms and propositions could be evaluated.

In contrast, Leeds (2012) advanced and offered initial empirical evidence consistent with certain predictions derived from the theory of cognitive acuity. For example, data is provided demonstrating that the probability of respondents endorsing a response option as effective increases at a decreasing rate (i.e., a logarithmic function) as subject matter experts' ratings of that response option's effectiveness increase. Research across a wide variety of signal detection paradigms (e.g., detecting visual and auditory signals) finds a similar logarithmic relationship between the intensity of a signal's strength and its probability of detection by human observers, thus lending a degree of credibility to this proposition. Nevertheless, the theoretical framework is limited by several conceptual ambiguities. Perhaps most central, the meaning of a response option's "effectiveness signal" and its "signal strength" are not well explicated or defined. For example, it is unclear whether the signal strength of a response option should be interpreted as an intrinsic characteristic of that option, a respondent's interpretation of that option, or some combination of the two. As a result, what it means for a respondent to "detect" or fail to detect an effectiveness signal-and therefore how to interpret the nature of SJT choices-is difficult to define. From a cognitive processing perspective, Leeds (2012) also postulates that respondents choose their response to an SJT item by first determining the "absolute" effectiveness signal of each response and then performing all possible paired comparisons among those options to determine the winner. However, if respondents are able to identify the absolute effectiveness of a response option, an elaborate competitive comparison process would be unnecessary given that the rank ordering of response effectiveness is already known. In sum, the theory of cognitive acuity provides one of the few truly processoriented accounts of SJT responding. However, ambiguity among its core elements and how its primary mechanisms 
connect to fundamental psychological concepts from the cognitive and judgment and decision-making literature leave room for improvement.

\section{Theory of Situated Reasoning and Judgment (SiRJ)}

The preceding review highlights several important considerations that should be addressed in SiRJ as a general response process theory of judgment and decision-making in SJT responding. First, the theory should explicate the psychological processes involved in evaluating an SJT item. For example, for a standard multiplechoice SJT item such as that posed near the beginning of this paper, the theory should provide an account of the cognitive processes involved in interpreting the presented scenario, evaluating the available responses, and contrasting the effectiveness of those choices. The SiRJ framework addresses this demand by drawing from existing models and research on judgment and decision-making in the cognitive and decision sciences to develop a descriptive account of how individuals process information and formulate preferences when answering an SJT item. Second, the theory should explicate and be sensitive to how and why different individuals may respond differently to an SJT item. Research suggests that a person's choices on an SJT are related to their procedural and experiential knowledge (e.g., Lievens \& Motowidlo, 2016; McDaniel \& Nguyen, 2001; Motowidlo \& Beier, 2010; Stemler \& Sternberg, 2006; Schmidt \& Hunter, 1993). The SiRJ framework integrates the general conclusions from this line of research with theory on how expertise and experiential knowledge is reflected in human memory that provides a means for describing how and why individuals with different experiential bases may exhibit distinctive patterns of responding on an SJT. Finally, the theory should facilitate efforts to explain how changes in the structure of an SJT item or instruction set could influence respondents' judgments. For example, none of the previously reviewed conceptual frameworks suggest whether or how asking respondents to select the least effective response differs from selecting the most effective response on an SJT item. However, research examining differences in the predictive validity of SJT scores has revealed that respondents' accuracy at identifying the expert-keyed least versus most effective options exhibits different correlations with job performance (Stemler, Aggarwal, \& Nithyanand, 2016), suggesting that these responses may tap into unique aspects of judgment. The SiRJ framework addresses this need by proposing an integrative model of SJT responding that allows for both most and least effective responding to be represented; additionally, the cognitive mechanisms represented in SiRJ provide a foundation for characterizing how the structure and presentation of an SJT item may influence responding.

The sections below describe the response process mechanisms represented in SiRJ. Figure 1 offers a visual depiction and summary of the SiRJ framework and the proposed cognitive steps involved in responding to an 
SJT item. The model proposes three broad phases of SJT item responding, shown in the central gray circle of Figure 1: (I) reading and interpreting the item stem/situation, (II) reading and interpreting the available response options, and (III) selecting a response. A series of more specific cognitive processing activities are proposed to be carried out within each of these phases that can be broadly categorized into two types of judgment processes. The cognitive processing carried out during the first two phases of SiRJ (interpreting SJT item stems and response options) are proposed to reflect conditional reasoning judgments used to generate interpretations about situations and responses. The cognitive processing during the last phase of SiRJ (selecting final response) is proposed to involve similarity judgments and preference accumulation that indicate the desirability of potential responses to a situation. To facilitate explication of these processes and how they unfold within SiRJ, the response process model is broken down in the discussion below into seven steps corresponding to the numbered white boxes in Figure 1. The outcomes of each step feed into one another as part of an overall cognitive appraisal process. In this sense, SiRJ reflects a sequential/cyclical judgment and decision-making model consistent with information processing approaches adopted by many decision models in the cognitive sciences (cf., Oppenheimer \& Kelso, 2015; Weber \& Johnson, 2008) as well as narrative accounts of questionnaire responding (e.g., Ployhart, 2006; Tourangeau, Rips, \& Rasinski, 2000).

\section{Sequence of cognitive processing activities in SiRJ}

Step 1 proposes that individuals begin the SJT item evaluation process by constructing an appraisal of the situational context presented in the item. A typical SJT question contains an item stem conveying features about a scenario in which events are unfolding. For example, the item stem "You are shopping when you notice a man robbing the store" communicates that the individual is in a public locale, is witness to (but not necessarily the target of) a criminal activity, and the apparent gender of the perpetrator. From these characteristics, a respondent is proposed to infer the perceived needs/demands of the situation to be acted upon (i.e., "What is happening? Why is this happening?"). Notably, individuals may produce different interpretations of a situation based on the features to which they attend or recognize and their experiential knowledge (Rockstuhl et al., 2015). For example, whereas one respondent may interpret the situation as involving a physically imposing criminal that could threaten his/her personal safety, another respondent may focus on features that emphasize the act is occurring in a public location and thus others may be present to offer protection or assistance. This conceptualization thus assumes that situations can be defined by distinguishable characteristics, individuals may attend to and/or recognize different sets of these characteristics, and that interpretations of the situational context in which events are occurring is a function of which situational attributes are considered/recognized and the respondents' learned experiences. These foundational 
assumptions are widely supported by cognitive and neurological research on human memory, perception, and object categorization (e.g., Adolphs, 2002; Love, Medin, \& Gureckis, 2004; Medin \& Schaffer, 1978; Nosofsky, 1986).

Based on this characterization, the cognitive processing reflected in Step 1 of SiRJ more generally represents a respondent's attempts to formulate a frame of reference in which to evaluate the consequences and utility of potential responses. This recognition is noteworthy given recent propositions that the reasoning reflected in SJTs is "general" or situation-agnostic rather than context-dependent (cf., Lievens \& Motowidlo, 2016; Motowidlo, Crook, Kell, \& Naemi, 2009; Motowidlo et al., 2006a; Stemler \& Sternberg, 2006; Weekley \& Ployhart, 2005). In arguably the most direct examination of this claim, Krumm et al. (2015) compared respondent performance on multiple-choice SJT items that either contained (situation-present) or did not contain (situation-absent) situational descriptions. Respondents in the situation-present condition were presented with items structured exactly like the robbery SJT item presented earlier. However, individuals in the situation-absent condition responded to SJT items in which the situational description from the item stem was removed:

What would you do?

A. Leave the store as quickly as possible and call the police.

B. Try to apprehend the man yourself.

C. Follow the man and call the police as soon as he appears settled somewhere.

D. Do nothing as you do not wish to get involved in the matter.

Across two different SJTs and samples, Krumm et al. (2015) found that respondents selected the response keyed as most effective by subject matter experts regardless of whether the situation was presented on $43 \%-71 \%$ of items. The authors thus concluded that the provision of situational information in SJT item stems is largely inconsequential and that reasoning on SJT items reflects the application of broadly applicable general domain knowledge.

Though such findings seem to suggest that interpreting the situational context for an SJT item need not occur, SiRJ offers an alternative interpretation based on tenets of bounded rationality and the commonly observed finding that human judges restrict and/or frame decisions in ways that allow them to more easily reason through possible solutions (e.g., Kahneman, 2003; Klein, 1997; Simon, 1956). SiRJ proposes that SJT respondents can and do formulate a frame of reference by inferring a situational context from cues present in the item or test even if the situational circumstances are not explicitly provided (Harris et al., 2016). For example, it is posited that an individual presented with the situation-less SJT item about the store robbery presented above would infer from the response options alone that the context in which they are to interpret potential responses concerns witnessing a man engaging in an illegal act at a store (as opposed to, say, disciplining a subordinate or landing an airplane). Returning to the findings of Krumm et al. (2015), all respondents in these authors' original study were instructed that the SJT items 
they were completing concerned working in teams even though those in the situation-absent condition were not provided item stems describing specific team scenarios. SiRJ recognizes that even this type of innocuous cuing is sufficient for respondents to infer that the set of response options for a given item are not just randomly selected but are instead relevant to a commonly defined context. ${ }^{1}$

In sum, Step 1 of the SiRJ response process model posits that respondents formulate an interpretation of the situational context in an SJT item from available cues. For a typical SJT in which the items stems contain a situational description, individuals can rely directly on those situational features to derive the situational context (Rocksthul et al., 2015). However, even in atypical SJTs in which the items do not provide explicit situational descriptions, respondents can "reverse engineer" this frame of reference from cues in the response options or test instructions. This latter mechanism reflects the underlying assumptions that individuals taking a multiple-choice SJT (a) infer that a set of responses options for an item are related; (b) attend to cues in those options that imply this shared relation; (c) use those cues to infer the shared relation among responses; and (d) rely on this inference when considering the utility of possible responses. Importantly, this conceptualization does not imply "strict" contextdependent/situation-specific reasoning in the sense of $\langle\mathrm{IF}$ situation $\mathrm{X}$, THEN response $\mathrm{Y}\rangle$ (Stemler \& Sternberg, 2006). Rather it states that individuals attempt to constrain and bound the decision space when evaluating the utility of responses to an SJT item (Harris et al., 2016; Simon, 1956). Continuing the example of the robbery SJT item, a respondent may interpret the described situation as one in which the robber is armed, unlikely to be talked down, and that any attempts to flee the store or stop the larceny might result in harm to self or others. Note that none of these features are explicitly conveyed in the situational description for this item, but are potential extrapolations from the scenario based on one's experiential knowledge of robberies (e.g., actual lived experiences, stories heard from the news, events from television shows, etc.) that provide a defined context for interpreting potential responses.

Following Step 1, SiRJ proposes SJT respondents engage in a second conditional reasoning process in which the interpretation of the situational context elicits the test taker's likely response to the situation (Step 2) and the outcomes that result or are likely to result from that response (Step 3). There are two noteworthy assumptions regarding these cognitive mechanisms. First, an implicit understanding of the respondent's likely/preferred reaction to the situation is proposed to be elicited at this stage even if response options are provided (e.g., as on a multiplechoice SJT). However, it need not be the case that the test taker is explicitly aware of this response. Research on associative memory networks, values, and the elicitation of implicit beliefs have found that responses to and interpretations of an environmental stimulus frequently occur at levels beneath overt awareness (e.g., Freeman et al., 
2011; Greenwald et al., 2002; Nisbett \& Wilson, 1977). This does not mean individuals may not be able to articulate a spontaneously generated response if prompted; indeed, test takers can construct open-ended responses to an SJT item stem when asked (Motowidlo et al., 2006; Rockstuhl et al., 2015). Instead, these stages of the SiRJ framework maintain that when responding to an SJT item, test takers' interpretation of a situational context automatically and implicitly activates encoded goal-action pathways that reconcile the demands of a situation based on learned experiences (e.g., Stemler \& Sternberg, 2006).

A second point of note is that although presented as separate cognitive processing activities in Figure 1 for clarity, interpreting the expected outcomes achieved by a generated response (Step 3) is proposed to occur simultaneously—or so nearly as to be inconsequential—with the generation of that response (Step 2). In other words, respondents generate inferences about both "what action to do" (response) and "what will happen as a result of this action" (response context) concurrently when evaluating an SJT item stem. The intuitive and seemingly automatic connection between an individual's typical response and its anticipated outcome is a widely observed and defining feature of experience-based knowledge (Chase \& Simon, 1973; Lipshitz et al., 2001; Klein, 1997; Phillips et al., 2004). Thus, the exemplar respondent to the robbery SJT item may feel they would react to this context by attempting to safely hide themselves and any nearby others from the assailant (likely/preferred response) because it would minimize the chances of harm to self or others (likely consequences of action).

Having evaluated the situation and generated an associated response, respondents are proposed to move to a second phase of evaluation in which they interpret the response options provided on a multiple-choice SJT item. This process occurs in Step 4 of SiRJ and proposes that an individual generates an interpretation of the expected consequences for each of the $k$ response options provided for an SJT item based on (a) the features of the response option that are recognized or attended to and (b) the respondent's previously generated interpretation of the situational context. In simpler terms, this step proposes that individuals evaluate "What would be the likely consequences of response k given my interpretation of the situational context?" for each response option. This cognitive processing activity is identical to the previous step in SiRJ except that the interpretation is directed towards the anticipated consequences of the responses options provided for the item. Continuing the example respondent's evaluation of the robbery SJT item, the individual would consider the first response option and attempt to determine "What would likely happen if I tried to leave the store and call the police given that I suspect the robber is armed, dangerous, and a threat to me and others?" To the extent the respondent lacks direct knowledge about or experience with the actions and/or consequences of a given response option-which may be the case for many scenarios 
presented on SJTs - the individual is proposed to generate an interpretation by extrapolating from related knowledge or experiences. For example, perhaps the test taker has never previously attempted to safely escape from an armed robbery; however, they have tried to sneak out of a social gathering undetected or heard interviews of hostages fleeing their captors. Because these events share some features similar to those reflected in the SJT item, the respondent can use them to extrapolate the efficacy of this potential course of action (e.g., "I'm not very 'stealthy' and there are so many things that could prevent me from getting away, so I think it unlikely l'd be able to get out of the store"). Importantly, this conceptualization also offers a logical mechanism for how the same response might be interpreted differently across people and situations. For example, the efficacy of the response "Try to apprehend the man yourself" is likely to be interpreted differently by people with knowledge or experience physically subduing another individual. Similarly, this response option may be interpreted differently if one construes the situational context as involving a robber armed with a knife versus a firearm versus nothing.

The proposed judgment mechanisms summarized in SiRJ to this point describe the use of conditional reasoning processes to develop interpretations for the situation and available response options of an SJT item. The last phase of SJT responding in SiRJ describes how these interpretations are used by individuals to make their final response selections. In Step 5 of SiRJ, respondents are proposed to evaluate the similarity between the expected consequences of their generated response (from Step 3) and the expected consequences of each of the SJT item's response options (from Step 4). This cognitive processing mechanism reflects two important propositions supported by work in the broader judgment and decision-making literature. The first is that individuals consider the anticipated consequences of possible SJT response options rather than the extent to which each response option is similar to the actual behaviors the test taker has done or observed in the past. In other words, this stage posits that the example respondent completing the robbery SJT item would not focus on which of the available response options is most like hiding from the robber (i.e., the individual's likely/preferred response based on the situational context), but rather which of the response options is more likely to minimize the chances of harm to self or others (i.e., the expected outcome of the individual's likely/preferred response). The claim that individuals attend to the expected outcomes of decisions is consistent with most existing work on human judgment and reasoning. For example, contemporary theories and research in social cognition and motivated reasoning construe the potential actions a decision-maker could take as means for satisfying one or more ends. From this perspective, behaviors are defined by attributes such as their value, expectancy, and instrumentality that reflect their propensity for achieving particular outcomes (e.g., Kruglanski et al., 2002; Vancouver, Weinhardt, \& Schmidt, 2010). Asking test takers to endorse SJT 
response options based on their perceived effectiveness can similarly be conceptualized as a series of means-ends evaluations that individuals attempt to resolve/optimize by considering "Is this action likely to result in an outcome I perceive as desirable given my interpretation of its characteristics and the situational demands?" This proposition is also consistent with Motowidlo and colleagues (Motowidlo et al., 2006a; 2006b) discussion of implicit trait policies and Stemler and Sternberg's (2006) conceptualization of "practical intelligence" that both posit individuals endorse SJT response options based not on the behavioral actions they express, but rather their perceived effect and whether it satisfies the goals/beliefs they wish to achieve in that situation.

In addition to these theoretical justifications, there is also some empirical evidence that SJT respondents focus on the behavioral consequences of response options. For example, Krumm et al. (2015) analyzed respondents' verbalized thought processes while completing situation-absent SJT items. Even in these context-less items, the authors found that test takers reported describing the feasibility, efficacy, fairness, and motivating effects of the available response option behaviors (i.e., their expected consequences) rather than whether the response options were similar to specific actions they had carried out or would be likely to carry out. Assuming that individuals infer, rely upon, and compare the perceived consequences of their likely response to those of the response options also offers an intuitive explanation for how a respondent is able to evaluate-and potentially even endorse as highly effective-a behavior they may never have considered in a situation they may never have experienced. Attempting to infer how a novel or unexperienced behavioral response might "play out" in a scenario enables individuals to evaluate the extent to which potential actions could result in outcomes similar to those they desire. Research on naturalistic decision-making finds that such "mental simulation" and comparison against the results of known alternatives is among the most commonly observed ways in which individuals evaluate the efficacy of new and/or unexpected courses of action (Klein, 1997; Phillips et al., 2004).

A second key proposition reflected in Step 5 of SiRJ is that perceptions of similarity between the expected outcomes of a generated response and that of a given response option serves as an indicator of effectiveness. In support of this mechanism, a key conclusion from Motowidlo and colleagues' (e.g., Motowidlo et al., 2006a; 2006b; Motowidlo \& Beier, 2010) line of research on SJT responding is that test takers' perceptions of response option effectiveness is based on the extent to which they believe the action reflects the way in which they tend or prefer to behave. Seminal research on similarity judgments from cognitive psychology report that perceptions of similarity are sensitive to and only interpretable within the context of a defined criterion (e.g., "similar with respect to what?", Medin, Goldstone, \& Gentner, 1993; Tversky, 1977). Integrating these two sets of findings, the SiRJ framework proposes 
that the expected outcomes of a respondent's likely/preferred response serves as the point of reference against which the effectiveness of potential response options can be evaluated. Thus, if the response option "Leave the store as quickly as possible and call the police" is perceived by the respondent to result in outcomes similar to hiding from the assailant (i.e., the respondent's likely/preferred action based on their interpretation of the situation), the individual should perceive this option to be at least partially effective. However, the respondent may perceive the option "Do nothing as you do not wish to get involved in the matter" as more effective if they judge its consequences as more similar to those associated with attempting to hide.

Once similarity judgments for each response option are made, Step 6 in SiRJ proposes that these evaluations are compared against an internal threshold representing the strength of evidence required by a respondent to conclude a potential response option should be selected. This threshold is conceptually similar to decision criterion parameters in other general decision-making models involving evidence or preference accumulation (Busemeyer \& Townsend, 1993; Oppenheimer \& Kelso, 2015; Weber \& Johnson, 2008) and may vary as a function of the perceived importance of the decision (e.g., more important/consequential decisions require more evidence) or other dispositional traits (e.g., lower tolerance for ambiguity requires more evidence). If a single response option for an SJT item surpasses the threshold, then the respondent is proposed to select that response as the preferred option (Step 7). However, if no options or multiple options exceed the threshold, the individual is proposed to continue evaluating the item by returning to Step 1 of the response process. Should the evaluation process continue in this way, respondents are proposed to continuously aggregate (i.e., sum together) their preference strengths for each response option over time until either a single response option accumulates enough support to surpass the decision threshold or the respondent's time/motivation to deliberate is exhausted.

Unlike previous conceptualizations of SJT responding (e.g., Leeds, 2012; Motowidlo et al., 2006a; Ployhart, 2006; Stemler \& Sternberg, 2006), these final mechanisms in SiRJ reflect that individuals may need to evaluate the information presented in an SJT item multiple times in order to make a response selection. Furthermore, it is proposed that test takers will consider the entirety of the SJT item (i.e., both the item stem/situation and available response options) as part of this re-appraisal. Although it is possible that test takers could "skip over" parts of an SJT item during subsequent evaluations (e.g., not reevaluate the situation/item stem again), there is currently no theoretical or empirical evidence to suggest if or under what circumstances this is likely to occur. A unique feature of this assumption though is that it naturally accommodates the equifinal and multifaceted nature of complex decision problems characteristic of many SJT items. Notably, the representation of SJT responding as a cascading sequence 
of cognitive processing activities means that different conclusions at any step during the reappraisal process can result in different interpretations/preferences that the individual must now resolve or accommodate. For example, perhaps our exemplar respondent initially perceived "Do nothing as you do not wish to get involved in the matter" as the most effective behavior given their first read of the situation and response options, but they do not find it a fully satisfactory response. Consequently, the individual reevaluates the item stem and this time construes that the perpetrator in the situation may not be armed or physically imposing. This alternative frame of reference now renders a different set of responses plausible that results in the respondent perceiving "not getting involved" as still somewhat effective; however, "attempting to stop the robber" is also now a much more attractive response than previously considered. Given this possibility, these new appraisals are combined with the respondent's initial interpretations to update his/her perceptions of each response option's effectiveness. As this process plays out, the cumulative effectiveness and preferential ordering of response options may change over time.

In summary, the general response process encapsulated in SiRJ describes SJT responding as a combination of conditional reasoning, similarity judgments, and information processing activities in which test takers construct and accumulate preferences for and against potential responses. A central assertion of SiRJ is that respondents interpret the perceived features, demands, and consequences of situations and responses when answering SJT items. The outcomes of these cognitive processes are a function of the learned knowledge and experience of respondents as well as contextual factors of the item (e.g., what response options are available, selecting most versus least effective responses) and testing environment (e.g., time demands, importance/significance of test performance, etc.). The preceding discussion of the SiRJ response process model provided a descriptive account of SJT responding in both a standard (e.g., situational item stem followed by multiplechoice response options) and a non-standard (e.g., situation-less items) test format. By adapting and building upon the core cognitive process mechanisms described in SiRJ, the theoretical model could potentially be extended to represent SJT responding for other SJT formats as well (e.g., providing open-ended responses versus selecting from available alternatives, Rockstuhl et al., 2015; rating the effectiveness of a single response option as opposed to selecting among multiple response options, Motowidlo \& Beier, 2010), though these are not considered further here.

\section{Computational Model of SiRJ}

The judgment and reasoning processes proposed in SiRJ are ubiquitous across many theories in the decision sciences, and efforts have been made to formally represent these mechanisms using computational modeling. A computational model uses logical statements and/or functional equations to describe the processes 
responsible for system behavior over time (Harrison, Lin, Carroll, \& Carly, 2007). Translating natural language theories of phenomena into formal computational models involves developing and justifying a set of plausible algorithms and/or mathematical specifications of how variables and events influence one another and dynamically unfold over time (Miller \& Page 2007). A key advantage of implementing theory into a computational model is that it provides the means to probe the logic, consistency, and sufficiency of the theory's core concepts and mechanisms through virtual experimentation and simulation (Davis, Eisenhardt, \& Bingham, 2007; Epstein, 1999; Kozlowski, Chao, Grand, Braun, \& Kuljanin, 2013; Vancouver \& Weinhardt, 2012; Wang, Zhou, \& Zhang, 2016). Consequently, the narrative description of SiRJ presented above was translated into a computational model.

Attempting to understand how a computational model operates by reading its equations and algorithms is a challenging and often ineffective means for inferring its core logic and intuitions (Somers, 2018; Wilensky \& Rand, 2015). To this end and to facilitate comprehension of SiRJ, an interactive online application was constructed that provides details on the computational framework and allows readers to learn about the model by exploring its operations hands-on (https://grandjam.shinyapps.io/siri). Using a series of interactive inputs and graphics, key parameters of the model are described and can be manipulated in real-time to examine their effects in a small-scale simulation of SiRJ. The online application provides the most detailed account of the foundational components, assumptions, and operationalizations of SiRJ's computational architecture. A high-level and non-technical summary of the model processes is provided below for readers uninterested in the computational details. Appendix A offers a "middle-range" description of the initialization and computational procedures carried out in the SiRJ model.

\section{Model Operationalization and Functioning}

Much of the computational architecture for SiRJ builds upon a previous computational model and theory of diagnostic hypothesis generation (HyGene) by Thomas, Dougherty, Sprenger and Harbison (2008). HyGene provides a formal description of causal inference and prediction generation based on the interaction among episodic memory (stored experiences) and semantic memory (stored prototypes/knowledge) systems. Specifically, HyGene offers a process-level account of conditional reasoning rooted in theories of information processing and human memory to describe how people judge "Given what I've observed, what is the most likely explanation?" Thomas et al. (2008) demonstrate that HyGene is able to account for several empirical findings in the judgment and decisionmaking literature, such as the alternative outcomes (a person's judged probability of possible outcomes is sensitive to the strength of available alternatives) and subadditivity (probability estimates for "catchall" hypotheses tend to be lower than the sum of probability estimates that comprise the catchall hypothesis) effects. ${ }^{2}$ Furthermore, HyGene 
provides an account for how and why decrements to working memory capacity and environmental time pressures tend to impede the accuracy of judgments (Dougherty \& Hunter, 2003). Given that the form of conditional reasoning represented in the HyGene model is posited as a core mechanism in the judgment and decision processes used in SJT responding, it offers a robust, well-vetted, and process-oriented psychological theory and computational model upon which to build out the proposed item evaluation process characterized in SiRJ.

As summarized in Figure 1, SiRJ comprises a series of conditional reasoning judgments (Steps 1-4), followed by the formation of similarity judgments between the perceived consequences of provided response options and those of one's preferred response (Step 5), the accumulation of preferences for and against response options (Step 6), and finally the endorsement of one of the available response options (Step 7). Steps 1-4 of this item evaluation process are represented in the computational model for SiRJ as distinct iterations of the conditional reasoning processes described in the HyGene algorithm. Appendix A offers a descriptive example of how this process is carried out in Step 1 of the SiRJ model to generate an interpretation of the situational context based on the item stem given for an SJT item. The HyGene algorithm is also used in Steps 2-4 of SiRJ such that the outcomes of each conditional reasoning judgment serves as input for subsequent processing activities (e.g., Step 1: situational description in item stem used to generate interpretation of situational context; Step 2: interpretation of situational context used to generate respondent's preferred/likely response; Step 3: respondent's preferred/likely response used to generate interpretation of response outcome; Step 4: interpretation of situational context used to generate interpretation of expected outcomes for each response option).

Following these computations, Step 5 in the SiRJ model calculates the degree to which the expected outcomes of each available response option (outcome of Step 4 in SiRJ) match the expected outcome of the individual's generated response (outcome of Step 3 in SiRJ). This similarity judgment is represented as a correlation between these two response outcomes; consequently, a similarity evaluation is computed for each response option whose magnitude ranges from $[-1,1]$. A response option with a positive similarity judgment indicates that the anticipated outcomes for the response option are similar to the expected outcomes of the response the individual has most commonly associated with the perceived situation context, whereas a negative similarity judgment indicates the two are dissimilar. In Step 6 of SiRJ, the computed similarity judgment for each response option is compared against a decision threshold to determine whether it is sufficiently effective to warrant selection. The preceding operationalizations are unique in that they enable the SiRJ model to represent two distinct and parallel decision choices that respondents may be asked to make during SJT responding: identifying the most effective response 
option and identifying the least effective response option (e.g., Stemler et al., 2016; Weekley et al., 2015). Given the above interpretation for the directionality of the computed similarity judgments and the assumption that response options with outcomes more similar to those of the individual's preferred response are seen as more desirable (Motowidlo et al., 2006a; 2006b), a positively valenced threshold is used to represent the decision criterion for identifying the most effective response whereas a negatively valenced threshold is used for identifying the least effective response. The response option perceived as most effective is therefore defined as the option for which the similarity judgment exceeds the positive threshold, whereas the option perceived as least effective is the option for which the similarity judgment exceeds the negative threshold. Both decision choices were modeled in the present simulations. $^{3}$

If one (and only one) response option exceeds the threshold indicating high response effectiveness and one (and only one) response option exceeds the threshold indicating low response effectiveness, those options are selected as the most and least effective responses, respectively, and the item evaluation process concludes (Step 7). If no options have exceeded either threshold, multiple options have exceeded either threshold, or only a single threshold has been passed, the computational model returns to Step 1 and the entire item evaluation process repeats. If deliberation continues in this way, the similarity judgments calculated for each response option during each iteration are added together and thus accumulate over time until an unequivocal decision can be made or the simulated respondent exhausts their time/motivation to continue evaluating.

\section{Model Verification and Validation}

A strength of specifying theory computationally is the capability to evaluate whether its conceptual mechanisms "make sense," are sufficient to reproduce observed phenomena, and reveal unique insights (Davis et al., 2007; Epstein, 1999; Kozlowski et al., 2013; Wang et al., 2016). These goals are typically pursued through model verification and validation efforts (Naylor \& Finger, 1967; Sargent, 2013; Wilensky \& Rand, 2015; Van Horn, 1971). Model verification focuses on whether a computational model correctly corresponds with one's intended theory and conceptual model (i.e., does the model implemented in computer code faithfully recreate the proposed theory?). Model validation uses simulation and virtual experimentation methods to examine whether a computational model and its output possesses satisfactory correspondence with the "real world" based on its intended application (i.e., does the implemented model faithfully recreate patterns of observed effects for which it is applicable?). In practice, the simulations conducted as part of model validation often reveal insights, interpretations, and potential directions for future research/application. 
Owing to their diverse types and uses (Harrison et al., 2007), there is no standard criteria for verifying and validating computational models. However, several best practices have been recommended (Rand \& Rust, 2011). Table 1 summarizes these activities and how they were applied towards verification and validation efforts with the computational model for SiRJ. Model verification results are rarely reported in manuscripts as they tend to be conducted by the model developer while a model is under construction; in contrast, model validation activities are typically reported as they tend to be diagnostic of the plausibility and credibility of the conceptual theory and implemented computational model. Rand and Rust (2011) summarize four broad categories of model validation activity: micro-face validation, macro-face validation, empirical input validation, and empirical output validation. Of these, macro-face validation (extent to which model processes produce outcomes with sensible/face valid patterns irrespective of actual data) and empirical output validation (extent to which model processes produce outcomes whose patterns correspond with observed data) are particularly critical as they provide the most direct demonstration that the "micro-specifications" of a model are sufficient to generate the "macro-structure" of interest (i.e., the phenomenological results, Epstein, 1999). The foci selected to examine these two facets in the SiRJ model are summarized below. The specific targets associated with each of these model validation efforts are also summarized as research questions to facilitate presentation of the simulation results.

\section{Macro-Face Validation}

The selected foci for macro-face validation centered on two primary points of interest. The first concerns a fundamental proposition in SiRJ that differences in SJT response selection stem from differences in how individuals interpret the situations and responses presented on an SJT item and that these interpretations are informed by respondents' experiential knowledge (Motowidlo et al., 2006a; 2006b; Stemler \& Sternberg, 2006). The implication of this proposition is that individuals who tend to judge and react to events similarly should be more likely to make similar response selections on SJT items. This pattern is consistent with existing work that suggests individuals with similar personality characteristics/beliefs (Motowidlo et al., 2006b), from similar cultures (e.g., Lievens, 2006; Nishii et al., 2001), and those with similar training/background relevant to an SJT (e.g., Oswald et al., 2004) are more likely to respond similarly to SJT items. In SiRJ, these forms of experiential knowledge are reflected in the events and interpretations stored in a simulated respondent's episodic and semantic memory.

To examine this macro pattern, two parameters were incorporated into the SiRJ computational framework to manipulate the composition of individual's experiential knowledge between-person. The first, experience strength, was an ordinal scaling variable that controlled the size of a simulated test taker's episodic memory. A higher 
experience strength corresponds to an individual with a longer record of "lived" experiences relevant to the SJT content and therefore a larger store of events encoded in memory. This parameter had no effect on the diversity or content of experiences encoded in a respondent's memory; it simply resulted in some respondents having more of the same experiences stored in episodic memory. In contrast, the second parameter, expertise type, was a categorical variable used to produce between-person differences in the content of experiential knowledge. The term "expertise" is used loosely in this context as the simulation is agnostic to the meaning and nature of an expertise type and what distinguishes one type from another. More significantly, expertise type was designed to be analogous to Motowidlo and colleagues' (2006a; 2006b) characterization of implicit trait policies and Stemler and Sternberg's (2006) treatment of practical intelligence in that it represents a person's characteristic way of interpreting and reacting to events in the environment. Functionally, different expertise types correspond to different combinations of the situational context, response, and response context features associated with situations in memory (see Figure A1 in Appendix A). This operationalization resulted in simulated individuals with the same (different) expertise type tending to have similar (different) interpretations stored in episodic and semantic memory for the same events. By independently manipulating the "size" of episodic memory (i.e., experience strength) versus the content of episodic memory (i.e., expertise type), the simulation results can be used to examine the extent to which $\mathrm{SiRJ}$ is sensitive to differences in both facets of experiential knowledge.

RQ1: Do individuals with different experiential knowledge tend to respond differently to SJT items?

The second macro-face validation target concerns an additional core proposition in SiRJ that respondents consider the anticipated outcomes of the possible response options provided for an SJT item rather than the extent to which the behavioral actions reflected in those options are similar to the behavioral actions the test taker would likely do/finds preferable based on past experience (Step 5 of SiRJ). Though empirical evidence and conceptual frameworks were previously summarized that strongly support the proposition that individuals attend to the perceived consequences of SJT response options when evaluating their choices, a unique value of specifying theory computationally is the capability to examine and compare model behavior under different assumptions. An alternative version of the SiRJ model was thus constructed in which simulated respondents compared the degree of similarity between the preferred behavioral action (i.e., response) generated based on perceptions of the situational context to the behavioral actions described in the response options, rather than comparing the expected consequences (i.e., response interpretation) of those behaviors. This model specification effectively simplifies the SiRJ response process model shown in Figure 1 by removing Steps 3 and 4 and substituting comparison of expected consequences with 
comparison of behavioral actions in Step 5 (i.e., "Is this action behaviorally consistent with my likely/preferred action?"). All the remaining model processes were held the same. Contrasting the output of SiRJ under these different levels of complexity offers a means for evaluating whether and how the computational model is sensitive to differences in the proposed cognitive processing mechanisms employed during SJT responding. ${ }^{4}$

RQ2: Does SJT responding differ when judgments are based on the similarity between the behavioral action described in SJT response options and the behavioral action of a respondent's likely/preferred response versus when judgments are based on the similarity between perceptions of the anticipated consequences of SJT response options and the perceptions of the anticipated consequences of a respondent's likely/preferred response?

\section{Empirical Output Validation}

The foci for the empirical output validation tests centered on the potential for SiRJ to account for and generate SJT response patterns consistent with those of real respondents. Importantly, the empirical relationships of interest for this purpose are not the same as those most commonly reported in the SJT literature (e.g., correlations of SJT performance with job performance or other individual differences). Instead, the most meaningful points of comparison are data examining how individuals evaluate SJT items and which response options they endorse. The extent to which SiRJ adequately reflects these patterns lends confidence to the conclusion that the core process mechanisms specified in the formal model are plausible and sufficient representations of SJT responding.

Two such patterns of responding were gleaned from the SJT literature as useful empirical output validation comparisons. The first involves the previously discussed findings from Krumm et al. (2015), in which respondents completing either situation-present or situation-absent SJT items (i.e., situational description in the SJT item stems was provided versus not provided, respectively) frequently chose the same response options as most effective. Krumm et al. (2015) concluded from this result that the provision of situation specific information does not play a significant role in SJT judgments. As discussed previously, the response process model proposed in SiRJ offers an alternative conceptualization in which individuals formulate a frame of reference in which to evaluate potential response choice judgments, even in the absence of a situational item stem. In this regard, SJT responding is not

proposed to reflect "strict" context-dependent reasoning that requires a situational description (i.e., IF <situation X>, THEN <response $Y>$ ), but it does reflect a form of bounded conditional reasoning. If this foundational cognitive process in SiRJ characterizes the judgment and reasoning mechanisms involved in SJT responding, the computational framework should be capable of producing an effect pattern similar to that reported in Krumm et al. 
(2015). To examine this claim, a set of conditions were constructed in which the simulated SJT items were presented with (situation-present) or without (situation-absent) situational descriptions. In the situation-present condition, simulated respondents made use of the provided situational description to interpret the situational context in Step 1 of the SiRJ response process. In the situation-absent condition, simulated respondents instead used the response options provided in the SJT item to interpret the situational context. Evaluating the extent to which simulated respondents across these two scenarios endorse similar response options comparable to the participants in Krumm et al.'s (2015) studies provides a useful diagnostic test of the SiRJ response process model's generative sufficiency.

RQ3: Does the provision of situational information influence which responses are selected on SJT items?

A second target identified for empirical output validation concerns the nature of response instructions that ask test takers to identify the most versus least effective responses for an SJT item. Most studies examining SJT responding have tended to ask respondents to identify what they would do given a set of circumstances or to select the response perceived as most effective. In both cases, the presumption is that this response reflects something about the individual's most comfortable, natural, or learned behavioral tendencies (Motowidlo et al., 2006b; Motowidlo \& Beier, 2010; Stemler \& Sternberg, 2006). Although less attention has been directed towards the interpretation of least effective responding, the above logic suggests such that this response reflects the opposite information (i.e., what is least comfortable, most unnatural, or most antithetical to learned tendencies).

Brooks and Highhouse (2006) proposition that SJT responding taps into "quasi-rationality," or the balancing of intuitive and analytical reasoning in an individual's decision-making, offers one potential lens through which to construe the nature of most and least effective responding in SJTs. There is a rich body of research indicating that judgments characterized by greater intuition versus greater analysis tend to exhibit different properties (Hammond, 1996). Specifically, more intuitive judgments tend to involve less cognitive control, faster rates of data processing, and less conscious awareness of the method used to reach a conclusion, whereas more analytic judgments involve higher cognitive control, slower rates of data processing, and more conscious awareness of the deliberation process. Given this conceptualization and the belief that selection of the most effective responses to an SJT item corresponds with differences in a respondent's natural preferences and experientially reinforced tendencies, judging the most effective response for an SJT item appears consistent with judgments characterized by intuition. In contrast, identifying the least effective SJT response appears more analytical given that it requires reasoning beyond one's normal way of responding to a situation. Consequently, the speed with which these two response judgments are made may offer a useful diagnostic test for examining the fidelity of SJT responding in SiRJ. To investigate these 
potential response patterns, simulated respondents were tasked with selecting both the most and least effective response options for each item. No modifications were required to the model specifications of SiRJ to explore these potential differences in responding. However, no published empirical observations on the speed of most versus least effective SJT responding were found in the literature to serve as a point of comparison. Consequently, a new dataset was collected to examine whether (a) this difference exists in observed SJT responding and (b) the SiRJ computational framework produces differences in the speed at which respondents make most and least effective response selections.

RQ4: Does the speed with which most versus least effective responses are identified on SJT items differ? In sum, the model validation targets examine key assumptions of the underlying conceptual theory and implemented computational model of SiRJ as well as the extent to which its proposed processes generate effects consistent with real SJT respondents. Exploring the associated research questions also has the potential to suggest new insights and possible areas for future research, development, and application. A simulation study designed to evaluate these research questions and validation foci is described below.

\section{Method}

The implemented computational model for SiRJ contains parameters used to initialize three domains: (1) the person, (2) the SJT, and (3) the environment. The person parameters determine the attributes of simulated individuals, including their memory systems and decision criteria. The SJT parameters control the design and construction of the simulated SJT. Lastly, the environment parameters establish the situational ecology in which test takers were presumed to exist and be sampled from. Appendix B describes the entire set of model parameters in SiRJ associated with each of these domains as well as the specific values used in the present simulation. Given the size of this parameter space, it was neither necessary nor beneficial to conduct virtual experiments in which all (or even most) of these parameters were manipulated. ${ }^{5}$ Instead, the simulation design and manipulations were selected to specifically examine the primary research questions targeted for the model validation tests.

\section{Simulation Parameters}

Person. The person parameters manipulated in the simulation study were the previously described experience strength and expertise type of respondents that dictated the size and composition (respectively) of an individual's episodic memory. Although the SiRJ model allows any number of levels to be manipulated for both factors, the decision was made to restrict experience strength to four and expertise type to five categories. These parameter ranges were chosen to allow sufficient between-respondent variability in episodic memory structure while 
keeping the total number of simulation runs needed to produce a reliable pattern of results manageable. Aside from these parameters, all other person-level parameters were held constant across simulated respondents.

SJT. The simulated SJT contained 20 items each with four response options. The procedures used to construct the simulated SJT items attempted to mirror those employed in real SJT design. Specifically, the process used to develop situational descriptions for each simulated SJT item stem involved sampling unique scenarios relevant to a set of higher order dimensions/classes. For example, Oswald et al. (2004) describe an SJT intended to measure non-academic indicators of college performance. To construct their SJT item stems, the authors identified twelve broad dimensions (e.g., perseverance, social responsibility, continuous learning) and then developed specific scenarios relevant to each of those domains to use as the situational description for each SJT item. A similar logic was used to construct the item stems for the simulated SJT, though the simulation was agnostic to the actual nature of item content. Each simulated SJT item was assigned to correspond with one of the three broad dimensions/classes constructed as part of the environmental ecology of respondents described below (e.g., Item 1 dimension = perseverance; Item 2 dimension = social responsibility; Item 3 dimension = continuous learning). The number of simulated items per dimension was determined randomly such that each broad situational class was represented, but there was variability in the number of items per dimension. ${ }^{6}$ Lastly, the final scenario used for each item stem was determined by randomly selecting and then slightly altering one of the five specific situations from the environmental ecology associated with the broad situational class assigned to an SJT item (e.g., broad dimension for Items 1 and 5 = perseverance; Item 1 item stem = completing a task even when it is not interesting; Item 5 item stem $=$ working towards a long-term goal).

The response options available for each item were similarly constructed by randomly selecting and then slightly altering possible responses to each situation across the five expertise types represented in the simulation (see Panel B of Figure A1, Appendix A). Constructing the response options in this way was intended to mirror that of critical incident approaches in which plausible responses to a situation are solicited from subject matter experts, incumbents, or other individuals with varying backgrounds and expertise so that an array of possible behavioral reactions are represented on the assessment tool (McDaniel \& Nguyen, 2001; Oswald et al., 2004). In this sense, response option A for the first simulated SJT item could be construed as representing a behavioral response solicited from one type of expert (e.g., students enrolled in a university's honors college) whereas response option B might reflect a behavioral response solicited from a different type of expert (e.g., transfer students from a community college). 
The same SJT items were administered to all simulated individuals to ensure that response patterns could be compared across the entire sample. Although the specific items used in the simulation could have idiosyncratic effects (e.g., SJT happens to contain items stems and/or response options not well represented in the experiential repertoire of simulated individuals), the overall trends and general conclusions are unaffected if a different SJT is simulated using the same test construction procedure.

Environment. A procedure similar to that used by Thomas et al. (2008) was used to construct simulated respondents' memories. An environmental ecology consisting of three broad situation classes (e.g., perseverance) each containing five types of situations specific to that class (e.g., completing a task even when it is not interesting, working towards a long-term goal) was constructed. This resulted in a total of 15 specific situations that could be experienced and encoded in a simulated individual's episodic memory. The rarity of each specific situation in the environment—and thus the likelihood that a simulated individual may have encountered it—was represented by a probability distribution associated with each broad situation class (see Figure B1, Appendix B). The parameterization of these distributions was such that (a) the likelihood of experiencing any given specific situation was similar across all simulated individuals and (b) there were no cases in which a simulated individual did not have at least one experience with each specific situation. All other parameters used to generate the environmental ecology were held constant across simulated individuals. This operationalization thus assumes that all the simulated test takers were sampled from the same environmental background and had similar opportunities to experience each situation.

\section{Simulation Design}

The experience strength (4) and expertise type (5) parameters were fully crossed between respondents to generate 20 conditions in which the knowledge and experiential structure of simulated individuals differed. These manipulations permit direct examination of RQ1 and RQ4. In addition, two within-respondent manipulations were included to investigate RQ2 and RQ3. The first factor determined whether the simulated respondent relied on the expected consequences (response context) or the behavioral content (response behavior) of response options when judging their effectiveness. The second factor sought to reproduce the conditions described in Krumm et al. (2015) and controlled whether the respondent completed a situation-present (all SJT items contained an item stem with four response options) or situation-absent (all SJT items contained only four response options but no item stems) version of the SJT. Manipulating these parameters within-respondent meant the "same" respondent participated in all four unique combinations of these parameters. However, observations across these conditions were completely independent. In other words, four identical "clones" of each simulated respondent were constructed and completed 
the SJT under each combination of the within-respondent parameters as though it was the first time they had encountered the items. This design choice is unique in that it removes any idiosyncratic person-level sources of variance across simulation conditions. As a result, it affords an exact counterfactual comparison to evaluate the impact of SJT item stem removal (RQ2) and differences in the proposed response evaluation mechanism (RQ3) on SJT response selection that would be impossible to achieve with real human respondents.

The final simulation thus employed a fully crossed 4 (experience strength, between) $x 5$ (expertise type, between) x 2 (item stem, within: situation-present, situation-absent) x 2 (evaluation criterion, within: response consequences, response behavior) mixed factorial design. A total of 10,000 unique respondents were created (i.e., 500 for each unique combination of experience strength $\mathrm{x}$ expertise type), resulting in a total of 1.6 million simulated SJT choices (10,000 respondents x 20 SJT items $x 2$ response selections per item [most effective and least effective] $\mathrm{x} 4$ within-respondent conditions). Code for the model, simulation, and analyses were created and conducted in $\mathrm{R}$ Version 3.5.0 (R Core Team, 2018). The reported model simulations were run on the University of Maryland's high performance computing cluster using $\mathrm{R}$ version 3.3.2. All code needed to run the simulations and analyses as well the full simulated dataset reported in the paper are available at https://osf.io/uwdfm/.

\section{Simulation Measures}

Two primary outcome variables were recorded in the simulations to evaluate the proposed research questions: (1) the response options selected as most and least effective by simulated individuals for each item, and (2) the number of times simulated individuals iterated through the item evaluation process in SiRJ before identifying their preferred most and least effective responses. Although SiRJ does not produce true response times (e.g., seconds required to make response selections), more iterations through the item evaluation process corresponds with a simulated individual deliberating longer over which responses to select and thus the extent to which respondents take more or less time to make their final choices.

\section{Empirical Data Collection}

As noted previously, no existing empirical data sources reporting response speed differences in the selection of most versus least effective responses for SJT items was found in the literature to serve as a point of comparison for examining RQ4. A new data source was thus collected for this purpose. The reported data collection was reviewed and received approval from the University of Maryland-College Park IRB (Project \#853978-2: Evaluating patterns of responding on situational judgments). 
Participants. Data were collected from 132 undergraduate students from the University of Maryland who volunteered for either course credit or $\$ 10$. Data from seven participants were removed from the final dataset due to equipment issues, resulting in a final dataset of 125 participants. The mean age of participants in the sample was 20.25 with a demographic breakdown of $69 \%$ female, $28 \%$ White/Caucasian, $20 \%$ Asian/Asian American, and $20 \%$ Black/African American.

Equipment. The response time data used to examine RQ4 were collected as part of a separate data collection conducted by the author using an eye-tracking system and software platform (SR Research EyeLink, 2009) to monitor respondent's attention during SJT responding. SJT items were presented to participants on a computer screen. Each item contained an item stem with a situational description and four possible response options. Item responses were made by using a mouse to click the desired response option on the screen, with the mouse position reset to the middle of the screen at the presentation of each SJT item.

SJT items were presented on screen one at a time. Participants were provided written instructions on the computer screen before and during the presentation of each item indicating whether they should select the response they perceived as most or least effective for that item (but not both). Thus, unlike the simulated respondents in SiRJ, participants in the empirical study did not report their most and least effective response choice for each SJT item. However, this procedure allowed the speed of responding for these two response choices to be isolated and independently measured for participants. The amount of time (in milliseconds) required to choose a response for each item was computed by the eye-tracking software as the time between when an SJT item first appeared on screen to the time the participant clicked their response.

SJT. The SJT contained 36 items drawn from a larger pool of items constructed and validated by Oswald et al. (2004) to measure alternative (i.e., non-academic) dimensions of college performance in undergraduate students. This SJT was selected because the content and situational circumstances reflected in the items were germane to the study population of undergraduate participants. As such, it was expected that respondents would be likely to possess experiential knowledge relevant to making response selections.

Procedure. Participants were scheduled to complete the SJT individually and in-person under the supervision of a trained experimenter. The SJT was delivered in a dedicated room containing all the eye-tracking, computing, and data recording equipment. Once a participant arrived in the lab space, the study procedure and purpose was fully explained and consent obtained. Participants were informed they would be reading a series of 
scenarios accompanied by four potential responses and they would be asked to select either the response they believed was most effective or the response they believed was least effective for each situation.

Following calibration of the eye-tracking equipment, participants viewed and responded to a practice SJT item with instructions about which type of response they should select to familiarize themselves with the procedure. The instructions presented to participants regarding whether to select the most or least effective response alternated between items (e.g., select most effective response for the first item, least effective for the second, most effective for the third, etc.). To control for potential order and fatigue effects in the analyses, the order in which SJT items were presented and the order of response type selection were counterbalanced. This resulted in four experimental conditions to which participants were randomly assigned. Table 2 provides a summary of the experimental design and the final number of respondents per condition. The response time required by participants to complete each item was recorded as the primary variable of interest in the study.

\section{Analysis Strategy}

RQ1-RQ3 consider whether the specific parameters manipulated in the simulation result in different patterns of response option selection. The outcome of interest for these questions is not a single output variable (e.g., a total performance score), but rather the specific sequence of most and least effective response choices chosen by simulated respondents across the 20 SJT items. For example, the response options selected as most effective by a respondent on the first five SJT items might be $\{A, C, A, D, B\}$ and least effective $\{B, A, B, C, D\}$. This entire response pattern can be summarized by a single concatenated response vector: $\{[A, C, A, D, B],[B, A, B, C, D]\}$. Consequently, RQ1-RQ3 ask whether changing the experience strength, expertise type, evaluation criterion, and item stem presence in SiRJ result in simulated respondents producing different response vectors.

To provide an overall assessment of these questions, a neural network classification model was trained and evaluated using the response vector data generated by simulated respondents. Neural network classification models are a class of supervised machine learning techniques that take a set of input values and attempt to identify a unique set of weights that can be used to classify stimuli possessing different combinations of those input values as belonging to one or more a priori identified output classes (Oswald \& Putka, 2015). For the present application, the neural network classifier was used to determine whether the response vector produced by simulated respondents (input values) could be used to (simultaneously) predict their experience strength, expertise type, whether they evaluated response options on the basis of behavioral actions or anticipated consequences, and whether they completed the situation-absent or situation-present SJT (output classifications). ${ }^{7}$ The extent to which the 
classification model is able to accurately predict the true values of each parameter based on the response vector indicates that manipulating the corresponding parameter in SiRJ tends to systematically change which response options simulated respondents endorse as most and least effective. Conversely, the extent to which the classification model is unable to predict respondents' true parameter values indicates that manipulating the corresponding parameter in SiRJ does not tend to produce distinctive patterns of response selection. Appendix $C$ provides further details on the specifications for the neural network classification model used in the present research.

A useful method for examining the results of classification models is to construct a confusion matrix indicating the number of times the model predicts a given attribute level for each known level of the parameter (e.g., how many times the classifier predicts Expertise Type 1 respondents as having Expertise Type 1, 2, 3, 4 or 5). A variety of evaluation metrics can be calculated from confusion matrix data to provide information on a classification model's predictive capabilities. The most common are precision (how often the classification model's predictions about a parameter level are correct), recall (how well the classification model is able to predict a given parameter level), classification rate (how often the classification model is able to make a definitive prediction), and overall accuracy (how often the classification model makes correct predictions overall). Separate confusion matrices along with these evaluation metrics were computed and examined from the neural network model's predictions for the experience strength, expertise type, evaluation criterion, and SJT type assigned to each simulated respondent to evaluate whether these parameters influenced SJT response selection in SiRJ. The average estimated classification probabilities for each parameter level were also examined (e.g., a more predictive model should assign a high classification probability to a respondent's true parameter value and low probabilities to all other values).

In addition to the quantitative analytic strategy described above, the results relevant to $R Q 3$ that examine response selections by simulated respondents in the situation-absent versus situation-present SJT conditions were evaluated in a semi-qualitative fashion to facilitate comparison with the results of Krumm et al. (2015). As a reminder, these authors observed that respondents taking a situation-absent or a situation-present SJT selected the same response option keyed as most effective by subject matter experts on $43 \%-71 \%$ of items. In the present study, there was no scoring key for the simulated SJT and thus no single response option selection to compare across these conditions. Instead, the degree of similarity in the full distribution of most effective response option selections between simulated respondents in the situation-absent versus situation-present conditions was compared. Evaluating the response selection distributions offers a similar point of comparison to that of Krumm et al. (2015), but also takes into account the relative degree of preference respondents in both conditions expressed towards all possible 
response options. Consequently, the distribution of most effective response selections were plotted and compared for both the situation-absent and situation-present conditions. To construct a comparable quantitative metric to that reported by Krumm et al. (2015), the proportion of items for which respondents in both conditions chose the most commonly selected response option was calculated. Additionally, Kendall's $\tau$ was computed using the rank order of response option selection frequencies on each item across conditions; the proportion of items for which $\tau>0$ was used as an additional indicator of the degree to which simulated respondents in the situation-absent and situationpresent conditions tended to express similar preferences regarding the most effective response option.

Lastly, RQ4 considers whether real and simulated respondents differ in the speed at which they make most versus least effective SJT response selections. This research question was examined using survival analysis techniques for both the empirical and simulated data. Survival analyses are a general set of statistical approaches for exploring the effect of predictors on time until occurrence of an event (Mills, 2010). In the present application, the event of interest was response selection for an SJT item and the key predictor is whether that response was for the most or least effective option. There are a variety of different survival analysis models that may be applied to event history data; for the present data, a parametric proportional hazards model using the Weibull distribution to represent time to SJT response selection was employed. This model reports the effect of potential covariates on the observed hazard rate (i.e., conditional probability that an event will occur at a particular time given that it has not yet occurred) in log-odds and thus allows an examination of how changes in a predictor variable influence the relative likelihood of an event occurring. The advantages of using survival analysis to examine the present research question is that it (a) permits computation of odds ratios for evaluating the effect of covariates on response speed across the simulated and empirical datasets; and (b) appropriately accounts for the effect of censored data (e.g., no response observed before observation period concludes) on model estimates. The former is relevant as it facilitates-though does not fully equate-comparisons between the simulated and empirical respondent data even though the response speed metrics are recorded on different scales (e.g., number of model iterations vs. milliseconds, respectively). The latter is relevant as preliminary analysis of the simulated response data indicated that not all simulated respondents had made their final response choices before the maximum number of item evaluation iterations permitted by the simulation was reached.

Given that the simulated and real response time data included multiple observations of SJT items nested within individuals, a two-level hierarchical Bayesian regression framework was used to account for both person- and item-level effects in the both datasets. In addition to the focal effect of response type (e.g., most or least effective 
response choice) on response speed, both regression models controlled for a number of covariates. Specifically, the model fit to the simulated data controlled for the presence of SJT item stems and the evaluation criterion used by the respondent. The model fit to the empirical data included word count (total number of words in item stem and response options) and the order in which the item was answered as covariates. Appendix $C$ provides additional details on the specification of the regression models used for both datasets.

\section{Results}

\section{Research Question \#1}

RQ1 examines whether differences in the experience strength and expertise type of simulated respondents in SiRJ result in the endorsement of different response options as most and least effective across SJT items. Tables 3 and 4 summarize the confusion matrix data from the neural network classification predictions for simulated respondents' experience strength and expertise type, respectively. To help visualize the simulation results, Figure 2 also plots the distribution of most effective response selections for a subset of the simulated SJT items across these parameters. ${ }^{8}$ Table 3 shows that the classification model was not successful at reproducing respondents' true experience strength on the basis of their response choices. Although the classifier was occasionally accurate at identifying respondents with Experience Strengths 1 and 4 on the basis of their response choices, it failed to classify $71 \%$ of observations overall and was unable to classify any respondents with Experience Strength 2 or 3 . The reason for these predictive failures can be gleaned from panel A of Figure 2. In general, response selection patterns were generally uniform within and between each level of experience strength, indicating that the experience strength parameter had no appreciable effect on response choices. Taken together, these results indicate that the number of events stored in a simulated test taker's episodic memory was not related to unique patterns of SJT responding in SiRJ. This observed pattern is sensible given that SJT response selection is not proposed in SiRJ (nor by other SJT researchers) to reflect differences in the amount of exposure to particular situations or responses per se.

In contrast, Table 4 reveals that the classification model was nearly perfect at reproducing respondents' true expertise type based on their response choices. As exemplified by the response distributions shown in panel B of Figure 2, respondents with different expertise types tended to make distinctive response selections. For example, on Item 3, respondents with Expertise Types 1, 2, and 3 tended to more frequently endorse Response Options A, B, and $D$ (respectively) as most effective, whereas respondents with Expertise Type 4 and 5 more frequently selected Response Option C. Additionally and with few exceptions, there also tended to be relatively high agreement within each expertise type regarding which response options those respondents perceived as most and least effective for 
each SJT item. Taken together, these findings indicate that changes in the content of simulated test takers' episodic and semantic memories resulted in distinct patterns of SJT responding. This pattern of results demonstrates a core premise of SiRJ consistent with propositions advanced in previous SJT research (e.g., Leeds, 2012; Motowidlo et al., 2006a; Stemler \& Sternberg, 2006)—differences in the SJT responses endorsed by individuals reflect differences in the learned beliefs, tendencies, and preferences a test taker holds about how to react in different situations. That is, the reason Figure 2B shows expertise type 1 respondents were more likely to endorse Option $A$ on Item 3 whereas Expertise Type 2 respondents were more likely to endorse Option B is because (a) the experiential knowledge of those individuals were qualitatively different and (b) those response options reflected different ways of reacting to a situation that were more consistent with how those individuals tended to behave. These analyses for RQ1 thus lend support to the macro-validation of the proposed process mechanisms in SiRJ.

\section{Research Question \#2}

$\mathrm{RQ} 2$ considers whether changing the evaluation criterion used by simulated respondents to formulate perceptions of response option effectiveness in SiRJ (e.g., comparing similarity in anticipated outcomes of possible responses versus similarity in their behavioral actions) results in different patterns of SJT response selection. The confusion matrix data from the neural network classification predictions for this parameter are summarized in Table 5 and reveal that the classifier was highly accurate at predicting which evaluation criterion simulated respondents were using based on the SJT options they selected. These results indicate that altering the core response evaluation mechanism in SiRJ tended to produce distinguishable patterns of responding across items. Figure 3 highlights these differences by presenting the distribution of most (panel A) and least (panel B) effective response selections for all the simulated SJT items across both levels of the manipulated evaluation criterion parameter.

These findings revealed two additional noteworthy observations. First, although the results of the neural network classification show that the evaluative criterion used by respondents in SiRJ led to sufficiently distinctive response selections, the most commonly endorsed response option was the same in both conditions for $45 \%$ of all observed responses ( $40 \%$ of most effective response selections, $50 \%$ of least effective responses; see shaded cells in Figure 3). In other words, if one were to look at only the most (or least) effective response made by a simulated respondent for a single item independently of their other choices, correctly identifying the evaluation criterion used to make that choice would be roughly a coin flip. This observation highlights the significance and importance of examining the pattern of response selections across multiple items (i.e., the response vector) when examining judgment and decision-making in SJT responding and their influencing factors. 
A second finding of note is that simulated respondents tended to exhibit slightly higher agreement regarding what was perceived as the most and least effective response to an item when using the similarity between behavioral actions as their evaluative criterion versus the similarity of anticipated consequences (i.e., tallest green bar (behavior) typically taller than the tallest red bar (consequences) in Figure 3). Though not shown, this effect was further pronounced when accounting for the expertise type of respondents in these response distributions (e.g., agreement among expertise type 1 respondents using behavioral similarity as evaluative criterion was substantially higher than among expertise type 1 respondents using anticipated consequences as evaluative criterion). To quantify this observation, the ratio between the number of simulated respondents selecting the most frequently endorsed response option when evaluating the similarity of behavioral actions relative to evaluating the similarity of consequences was computed for the most and least effective responses on each item within each expertise type. A ratio $=1$ on this metric indicates the same number of respondents in both conditions endorsed the most popular response, a ratio $>1$ that more respondents endorsed the most popular response when comparing behavioral similarity, and a ratio $<1$ that more respondents endorsed the most popular response when comparing similarity of consequences. Consistent with the qualitative patterns shown in Figure 3, the response option most commonly identified as most effective was chosen $62 \%$ more frequently on average (mean ratio $=1.62$, median $=1.56, S D=$ .42 ) and the response option most commonly identified as least effective was chosen $33 \%$ more frequently on average (mean ratio $=1.33$, median $=1.27, S D=.42$ ) when similarity of behavioral actions was used as the evaluative criterion versus similarity of consequences.

In sum, distinctive patterns of responding across the simulated SJT items emerged when simulated respondents relied on similarity of behaviors versus similarity of response consequences to guide their judgments. These findings indicate that the model mechanisms represented in SiRJ are sensitive to differences in the criterion respondents apply when evaluating the efficacy of potential SJT response options. However, the pattern of observed response choices within each condition was sensible and still consistent with the model's other core evaluative mechanisms (i.e., response choice strongly influenced by experiential knowledge of respondents), thus lending support to its macro-validation.

\section{Research Question \#3}

Turning towards the empirical output validation of SiRJ, RQ3 examines the extent to which the response selections of simulated respondents change depending on the provision of situational information in an SJT item or are consistent with Krumm et al.'s (2015) findings that SJT responding is invariant across these conditions. The 
confusion matrix data from the neural network classification predictions for the situation-absent versus situationpresent conditions are summarized in Table 6. Once again, the classification model was highly accurate at identifying whether simulated respondents completed a situation-absent or situation-present SJT based on the pattern of most and least effective response options endorsed. To provide a more direct comparison with Krumm et al.'s (2015) data that only examined the most effective choices of respondents, a classification model that included only simulated respondents' most effective response selections as the input to the neural network was also examined. The performance of this classification model was largely the same precision $=.92$, recall $=.95$, classification rate $=1$, accuracy $=.93$ ). Taken together, these findings indicate that manipulating the presence of SJT item stems in SiRJ tended to result in detectable differences in response selections. Figure 4 presents the distribution of most (panel A) and least (panel B) effective response selections for all the simulated SJT items across both conditions.

Although the neural network classifier was able to detect unique and subtle patterns of response pattern differences between situation-absent and situation-present respondents in SiRJ, the degree of response similarity across these conditions largely coincides with the results reported by Krumm et al. (2015). Recall that these authors observed that real respondents chose the same response as most effective on $43 \%-71 \%$ of items across situationabsent and situation-present SJTs. By comparison, the response option most frequently selected as highly effective was the same on both types of simulated SJTs for $55 \%$ of items (shaded cells in panel A of Figure 4). Additionally, $\tau$ was greater than zero-indicating items where the full rank ordering of response option selections by simulated respondents was similar in both conditions-on $55 \%$ of items. Both of these proportions are consistent with the empricial effects reported by Krumm et al. (2015) for responding on situation-present and situation-absent SJTs.

Beyond establishing correspondence with published empirical effects, the simulation findings examining $\mathrm{RQ3}$ revealed two additional observations. First, the degree of consistency in response option endorsement between situation-absent and situation-present SJTs tended to be stronger when simulated respondents selected the least effective response compared to the most effective response. Specifically, the response option most frequently selected as least effective was the same on both SJTs for $75 \%$ (shaded cells in panel B of Figure 4) and $\tau>0$ for $95 \%$ of items. Second, the presence of situational descriptions in the SJT item stems tended to result in a slightly but consistently higher proportion of simulated respondents endorsing the most frequently selected responses for a given item in SiRJ. Stated differently, there tended to be stronger agreement among simulated respondents about which response options were most and least effective when situational descriptions were provided compared to withheld. This effect is more readily observable when accounting for the expertise type of simulated respondents; Figure 5 
visualizes this pattern for the first ten SJT items (i.e., tallest green bar (situation-present) is typically higher than tallest red bar (situation-absent) for each SJT item). Calculating the same ratio as described in RQ2 for agreement in response selection by simulated respondents in the situation-absent and situation-present conditions, the response option most commonly identified as most effective was chosen $25 \%$ more frequently (mean ratio $=1.25$, median $=$ $1.15, S D=.30$ ) and the response option most commonly identified as least effective $14 \%$ more frequently (mean ratio $=1.14$, median $=1.08, S D=.24$ ) on average when the situation stem was provided versus when it was not.

Taken together, the pattern of results for $\mathrm{RQ3}$ indicate that the provision of situational descriptions in the SJT items produced detectable differences in the response options endorsed by simulated respondents in SiRJ. However, the degree of similarity in responding across these conditions was largely consistent with the effects reported by Krumm et al. (2015) and thus lend support to the empirical output validity of the model. The analyses also revealed distinctive patterns regarding the degree of consistency in and agreement among least effective versus most effective response selection when SJT item stems were present versus absent.

\section{Research Question \#4}

Drawing from the propositions of Brooks and Highhouse (2006), RQ4 considers whether the speed with which most versus least effective SJT responding differ. Table 7 presents the results of the hierarchical Bayesian survival analyses for both the empirical and simulated datasets. The parameterization of the survival model used in the present analyses was such that a positive coefficient indicates a variable tended to be associated with a faster response speed (i.e., larger hazard rate) whereas a negative coefficient indicates a variable tended to be associated with a slower response speed (i.e., smaller hazard rate). In proportional hazard survival models, it is often more informative to interpret the odds ratios for each regression coefficient rather than their estimated values (Mills, 2010). Odds ratios were thus computed by exponentiating each coefficient. These ratios can be interpreted as the relative likelihood that a one-unit change in the predictor is associated with a faster time to response on the SJT items.

The top half of Table 7 summarizes the survival analysis results using the response time data collected from real SJT respondents. These data showed a credible difference (i.e., credibility interval does not include zero) in participants' response times when making most versus least effective selections $(b=.175,95 \%$ credibility interval $[.088, .258])$. Controlling for the number of words and presentation order of the SJT item, asking respondents to choose the most effective response increased their likelihood of responding more quickly to an item by 1.19 times relative to when asked to choose the least effective response. In comparison, the posterior parameter estimates presented in the bottom half of Table 7 summarize the survival analysis results using the response speed data 
generated by SiRJ. Simulated respondents also produced quicker response times for most effective responding compared to least effective responding, though the difference was substantially larger than that observed in the empirical data ( $b=1.81,95 \%$ credibility interval $[1.80,1.83])$. Specifically, controlling for the presence of item stems and the evaluation criterion used, the likelihood that simulated respondents chose the most effective response more quickly was 6.11 times larger than when choosing the least effective response.

To further probe how this response selection process played out over time for individuals in SiRJ, Figure 6 provides an example of the item evaluation dynamics for a single simulated respondent for five SJT items. Each panel in this figure depicts how the simulated respondent's preference for each response option (in the form of cumulative similarity judgments, Step 5-6 of SiRJ) on each item accumulated during its deliberation. The times at which the simulated test taker identified its most and least effective response options are highlighted by the vertical dashed lines in each panel and indicate the number of complete iterations through the SiRJ item evaluation process that were required for the respondent to select each response. Note that if no response option emerged as the unequivocally preferred choice for either decision following the tenth iteration, the simulated respondent was forced to randomly choose an option for that decision.

Figure 6 demonstrates that there were times in SiRJ in which the simulated respondents could not definitively identify a single response option as its most or least effective on some items. For example, the response evaluation results shown for Items 1,3, and 5 in Figure 6 show that this respondent did not perceive any of the response options for these items as particularly poor actions (i.e., all response options had positive cumulative similarity and thus none exceeded the lower threshold for signifying a response as the least effective). As a result, this respondent was faced with the challenge of selecting the "worst among fairly good options" as least effective for these items. A different impasse is exemplified by the most effective response selections shown for items 1 and 4 in Figure 6. Although difficult to see on the graphs because their lines overlap, this simulated respondent perceived two response options as being equally highly effective (i.e., Response Options $A$ and $C$ in Item 1, Response Options $B$ and $C$ in Item 4). Consequently, the respondent was faced with the challenge of selecting the "better of equally excellent options" as most effective for these items. Both of these circumstances resulted in simulated test takers spending significantly more time deliberating over which response option to select.

In sum, the overall interpretation from the survival analyses conducted on the response time data from the empirical and simulated data are consistent with one another and reproduce effects in the same direction. The ordinal pattern of the response speed differences are further consistent with the conceptual proposition that selecting 
the most effective response option for an SJT appears more "intuitive" and occurs more rapidly whereas selecting the least effective response appears more "analytical" and occurs more slowly. Additionally, qualitative examination of the intra-item evaluation dynamics carried out by simulated respondents (e.g., Figure 6) revealed plausible circumstances and decision dilemmas that contribute to lengthier response evaluation times. Taken together, the results for RQ4 lend further credibility to the empirical output validity of SiRJ as a representation of SJT responding.

\section{Discussion}

Commenting on critical targets for advancing SJT research, Chan and Schmitt (2005, p. 238) noted "SJT performance clearly involves cognitive processes. [...] Addressing basic questions about these underlying cognitive processes and eventually understanding them could provide the key to explicating constructs measured by SJTs." Though others have echoed related sentiments (Brooks \& Highhouse, 2006; Lievens \& Sackett, 2007; Ployhart, 2006; Rockstuhl et al., 2015; Weekley et al., 2015), relatively little progress has been made towards developing a theoretical framework of these fundamental mechanisms underlying SJT responding. The motivation for the present work was to begin addressing this gap by proposing, formalizing, and evaluating a process-oriented theory of judgment and decision-making describing how individuals generate and evaluate responses on SJTs. The proposed response process model, SiRJ, draws upon and integrates existing work from the cognitive and decision sciences and offers an account of SJT item evaluation grounded in psychological research on memory, conditional reasoning, similarity judgments, and information processing. Specifying the SiRJ framework into a formal computational model further afforded the opportunity to explore its veracity against existing conceptual propositions and empirical effects. The model verification and validation efforts conducted with the model also revealed intriguing potential directions for future theory development, research, and practical application.

\section{Theoretical Implications and Future Research Directions}

Overall, the pattern of SJT responding observed from simulations with SiRJ was sensible, face valid, and consistent with empirical effects. Although no set of simulations or empirical comparisons can ever fully support the validity of a computational theory (Rand \& Rust, 2011), the model verification and validation efforts provide strong support for the plausibility of the theoretical process mechanisms represented within SiRJ. Consequently, it is justifiable to extrapolate from the simulation results and interpret these findings in more detail to stimulate further research into the judgment and decision-making processes involved in SJT responding.

Role of experiential knowledge in SJT responding. The results presented for RQ1 showed that differences in the way simulated respondents typically interpret and respond to situational demands produce 
qualitatively different patterns of SJT response option endorsement for both most and least effective choices. These results align with similar propositions previously advanced in the literature (Leeds, 2012; Motowidlo et al., 2006a; Stemler \& Sternberg, 2006). However, the representation of these interpretative differences as differences in the events and knowledge stored in episodic and semantic memory systems in SiRJ highlights the significance of more directly considering the cognitive structure of experience-based knowledge rather than the amount of experience held by a test taker when interpreting SJT performance. Prior SJT research has tended to examine measures of tenure, age, and/or job experience as a proxy for experiential knowledge when predicting SJT performance (e.g., Elliott, Stemler, Grigorenko, Sternberg, \& Hoffman, 2011; Weekley \& Jones, 1999; Weekley \& Ployhart, 2005). The simulation results from SiRJ show that amount of experience per se-in terms of the number of experienced events stored in episodic memory - was not predictive of SJT response selections. In this sense, SiRJ predicts that a more tenured test taker should not be any more or less likely to respond differently than a less tenured test taker if those individuals (a) have experienced even moderately similar events to those relevant for an SJT and (b) tend to interpret and respond to situations in a similar manner. For example, SiRJ would predict that an individual with a long history of hostility towards co-workers would likely respond similarly to an SJT concerning interpersonal skills as a brand new employee with a much shorter but qualitatively similar history of interpersonal hostility.

The effects of the experience strength and expertise type parameters on simulated respondents' knowledge structures were purposefully made independent in the present simulations to isolate their unique effects. However, attempting to represent correlations or interactions between amount of experience and the type of experiential knowledge held by respondents in future model and theory development could be fruitful. Doing so may open several potential avenues for research aimed at exploring how the unique knowledge gained through experience is cognitively represented, encoded, and acted upon in SJTs that moves beyond "more (less) experience is better (worse)" for SJT performance. For example, one hypothesis implicated by introducing a correlation between experience strength and expertise type in SiRJ is that the nature of SJT performance computed using expert- or consensus-based scoring keys would be expected to change in predictable ways based on the degree of experiential knowledge similarity between test takers and experts used to generate the scoring key. Grand, Allen, and Pearlman (2012) discussed some preliminary support for this pattern using a sample of junior Army non-commissioned officers taking a leadership SJT. In these data, SJT performance was observed to be higher for test takers whose military rank was closer to that of the senior officers used to construct the SJT performance key. Although military rank was related to length of job tenure in that sample, achieving a higher ranked officer position also necessitated meeting 
standardized training, proficiency, and performance criteria. Such findings are consistent with the general conclusion from SiRJ that individuals who "think more alike"-as reflected in their experiential knowledge—are more likely to respond to SJTs similarly. These results and those gleaned from the simulations with SiRJ suggest that examining the mental models, implicit associations, and learned heuristics of individuals used to construct SJT scoring keys could thus prove useful for interpreting and potentially scaling test takers' SJT responses.

SJT response evaluation criterion. A key goal of $R Q 2$ was to investigate the impact of a simplified response process in SiRJ by comparing response behavior when simulated test takers considered similarity between their preferred/likely response to a situation and the behavioral action reflected in the available response alternatives rather than deriving and subsequently evaluating similarity among the anticipated consequences of these elements (i.e., Steps 3 and 4 in Figure 1). The overall pattern of simulation results indicated that changing this evaluative criterion resulted in detectable response selection differences across the simulated SJT items. Nevertheless, the most and least preferred response selections were the same for $45 \%$ of the decisions made by simulated test takers, suggesting that this mechanism did not produce wildly divergent patterns of responding.

The simulation findings did, however, reveal a substantially higher degree of agreement regarding which response options were perceived as most and least effective when simulated respondents based their judgments on behavioral similarity. There are two explanations for this effect. Pragmatically, higher agreement was observed because the response options used in the simulated SJT tended to be fairly similar to those stored in simulated respondents' memory (see Appendix B). The degree of consensus observed in the condition in which simulated respondents evaluated the behavioral similarity of response options would have been somewhat more degraded had the response options used in the simulation differed more dramatically from those represented in simulated respondents' experiential memories. However, higher agreement also emerged because of the simpler recognitionbased form of conditional reasoning employed by simulated test takers in the behavioral comparison condition. In essence, this condition represented a respondent as making SJT choices based solely on answering the question "Have I acted like this in similar circumstances?" In contrast, simulated respondents that responded by evaluating perceived consequences engaged in an additional level of processing regarding the "downstream" impact of a response beyond the literal behavioral description. Consequently, idiosyncratic differences between respondents in those projective interpretations can lead to different perceptions of response option effectiveness and therefore reduce the likelihood the exact same response choice is endorsed. 
The pattern of results observed for RQ2 beg the question of which evaluative criterion-and, by extension, which item evaluation process_is a more plausible representation of real SJT responding. Though the present data do not offer a definitive conclusion on this point, there are at least two pieces of evidence that suggest the response process represented in Figure 1 in which respondents evaluate anticipated consequences is likely to be more representative of actual SJT responding. The first can be gleaned from the results of previous verbal protocol studies in which respondents were asked to communicate their thought processes aloud while completing SJT items (e.g., Krumm et al., 2015; Rockstuhl et al., 2015). Such data is sparse in the SJT research literature, but what is available suggests that individuals interpret, are aware of, and attempt to justify their SJT choices by considering the resultant effects of potential actions in a scenario. For example, many of the examples reported by Krumm et al.'s (2015; see their Table 3) verbal protocol analysis, such as "appreciativeness increases motivation" or "involving the whole team signals that everybody has a voice in decisions," point to a degree of higher-order reasoning regarding a response's utility that extends beyond test takers simply comparing whether a given response option is consistent with their previous behavior. However, such data cannot rule out the possibility that respondents could have generated such rationale post hoc after identifying whether a particular response was similar to their past actions.

A second line of evidence that appears to favor the evalution of anticipated consequences for SJT response selection can be extrapolated from the response speed data examined for RQ4. As noted previously, the magnitude of response speed differences between most and least effective response selection was sizable in the simulated data. The lower half of Table 7 also shows that these response time differences were even larger when simulated respondents attended to the behavioral similarity of response options rather than their anticipated consequences $(b=$ $1.20,95 \%$ credibility interval [1.19-1.22], odds ratio $=3.32$ ). Although not modeled statistically due to a lack of comparable conditions in the empirical data, Figure 7 plots the interaction between response type and evaluation criterion on response speed in the simulated data and demonstrates that the speed of most effective responding was strongly influenced by evaluation criterion used by simulated respondents. Given that the impact of response type (i.e., choosing most versus least effective options) on response speed was already notably larger in the simulated data relative to the empirical observations, the widening of this gap under the more simplified item evaluation process appears to provide further evidence against the empirical output validity of this mechanism. 
In sum, the simulation results suggest that unpacking the specific evaluation criterion employed by respondents when interpreting the efficacy of SJT response options may be a useful avenue for future research. The present simulations contrasted two plausible processes and revealed subtle yet diagnostic response patterns between them. This may suggest that future efforts to examine how the use of different evaluation criterion employed by SJT respondents influence choices could provide valuable insights into the nature of judgment during SJT responding. For example, comparing whether and/or how the choices made by respondents instructed to select the response option most (least) similar to actions they have done in the past versus the response option they perceive most (least) effective would offer a more direct test of the simulation conditions examined in the present paper. Similarly, continued efforts to pair SJT response data with verbal protocol data could further tease apart how the information respondents attend to informs and interacts with the evaluative criterion used to select the most and least effective response options for an item.

Context-dependent reasoning in SJTs. Some contemporary conceptual frameworks have posited that the reasoning captured in SJT performance reflects a form of generalized tacit knowledge and practical intelligence that span situations (Motowidlo et al., 2006a; Krumm et al., 2015; Lievens \& Motowidlo, 2016; Rockstuhl et al., 2015; Stemler \& Sternberg, 2006). One of the most provocative interpretations to emerge from these recent works is the claim that judgment on SJTs is not actually "situational" but rather context-independent. The response process theory proposed in SiRJ offers an alternative to this account, hypothesizing that respondents engage in a series of conditional judgments that inherently reflects a "weak" form of context-dependent reasoning in that a respondent's interpretation of the situation presented as part of an SJT plays a central but non-deterministic role in response choice. Consequently, a primary purpose of RQ3 was to determine whether the contextualized inference mechanisms in SiRJ could replicate the empirical effects reported by Krumm et al. (2015) that have been cited as support for context-independent reasoning in SJTs

The pattern of results summarized in Figures 4 and 5 highlight that the form of contextualized inference represented in SiRJ is sufficient to produce response patterns similar to the emprical effects documented by Krumm et al. (2015). Notably, however, the judgment mechanisms represented in SiRJ are rooted in well-established theories and research from the cognitive and decision sciences (e.g., bounded rationality, experience-based decision-making, hypothesis generation, Kahneman, 2003; Klein, 1997; Simon, 1956; Thomas et al., 2008) that posit decision-makers construct simplified frames of reference to orient their decisions and apply relevant experiential 
knowledge when evaluating possible alterantives. SiRJ also offers a precise account for why and how this contextualized frame of reference facilitates responding on SJTs—by serving as a diagnostic cue upon which experiential-based memory and knowledge are conditioned as individuals attempt to identify, generate, and interpret the plausibility of potential responses. Interpretation of the situational context for an SJT item establishes a respondent's decision space, or the domain of relevant judgments, interpretations, and responses that should be considered (Thomas et al., 2008). The significance of this operation is discussed frequently in models of naturalistic decision-making (Lipshitz et al., 2001; Lipshitz \& Strauss, 1997; Phillips et al., 2004) and its implications for SJT response selection are clear: if individuals differ in their interpretations of "what is happening" in a scenario presented in or implied by an SJT item, they can end up operating on different decision spaces that influence the perceived efficacy of available response options (Rockstuhl et al., 2015). Elaborating these processes and providing evidence of their generative sufficiency is thus a key contribution of the SiRJ framework to articulating the nature of judgment and decision-making in SJTs.

The above mechanism also holds implications for interpreting differences in the rate of agreement in response option endorsement between the situation-present and situation-absent conditions observed in the simulation. In general, simulated respondents exhibited consistently higher ambiguity regarding which responses were selected as most and least effective when information about the specific situation to which they were responding was withheld (situation-absent) versus when it was provided (situation-present). This pattern was not explicitly programmed into the computational model for SiRJ, but rather emerged naturally as a function of differences in the way in which respondents in these conditions formulated perceptions of the situational context. Respondents in the situation-present condition directly evaluated what they thought was occurring in an SJT item from the situational description provided in an item stem. In contrast, respondents in the situation-absent condition "reverse engineered" this information by indirectly inferring this situational context from the provided response options. This latter mechanism affords the potential for more diverse frames of reference to be generated by respondents in the situation-absent condition that subsequently contribute to the relatively lower observed rates of agreement. In other words, the size of the decision space generated by situation-absent respondents was less constrained, the potential repertoire of appropriate behavioral responses entertained was subsequently larger, and thus greater variability in response option endorsement could emerge.

Nevertheless, there was enough similarity in the decision spaces between situation-present and situationabsent respondents that they could often reach similar conclusions. A concrete example helps to clarify why this can 
be the case. Consider the contextual interpretation one might draw if an SJT item stem explicitly stated "You are witnessing an armed robbery" versus inferring that a set of response options involved witnessing some sort of illegal act. Although both interpretations would be likely to contain certain overlapping features/themes (e.g., addressing a violation of the law, personal responsibilities for enforcing regulations, beliefs about protecting oneself and others), the decision space encompassing "illegal acts" is larger and more diffuse than that of "armed robberies." As a result, the number of potential means one might consider for how to behave under the former interpretation will be more numerous and variable than under the latter.

This pattern of results closely corresponds with the large research literature on situational strength (e.g., Harris et al., 2016; Judge, \& Zapata, 2015; Tett, Simonet, Walser, \& Brown, 2013). Situational strength reflects the extent to which environmental cues are available to indicate the types of behaviors that are and are not desirable (Meyer, Dalal, \& Hermida, 2010). A consistent conclusion from this line of work is that strong situations with unambiguous cues enable individuals to better identify which and how certain behaviors lead to particular outcomes and thus more readily regulate their actions to achieve desired consequences. Although situation strength was not manipulated in the present simulations per se, providing versus withholding situational descriptions in SJT item stems arguably represents an extreme dichotomous manipulation of this variable (i.e., no versus some situational strength). An additional topic for future SJT research could thus be to attempt to replicate the patterns of agreement produced in SiRJ across situation-present and situation-absent SJTs, as well as explore the effect of more continuous manipulations of situation strength in SJT item stems on test takers' response patterns.

Most versus least effective responding. Although it is common for SJT respondents to be asked to select the responses they perceive as most and least effective for an item, there has been relatively little attention directed towards differentiating the nature of these two responses (Stemler et al., 2016). In SiRJ, identifying the most and least effective responses for an SJT item are represented as separate yet parallel decisions that unfold through repeated application of a single underlying judgment process. The simulation results and empirical data examining differences between most and least effective responding revealed a number of intriguing observations between these response types that suggest potential areas for future inquiry.

Figure 6 highlights that the process mechanisms in SiRJ led to emergent "decision dilemmas" during simulated respondents' deliberations that share plausible aspects with many real decisions. For example, identifying the "worst among good options" or the "better among excellent options" are decision scenarios commonly faced in 
the real world, and the manner by which individuals make these choices is frequently used to distinguish those perceived as having "good" versus "bad" judgment. However, making final SJT response selections when faced with these dilemmas also required the greatest amount of time and deliberation to resolve in the simulated results. Consequently, one hypothesis implied by these findings is that SJT items containing these dilemmatic choices would likely be perceived as more challenging and/or difficult to answer by real respondents (cf., Lievens \& Sackett, 2007).

The survival analyses conducted for $\mathrm{RQ} 4$ also revealed that both simulated and real respondents tended to consistently identify the response option perceived as most effective at a faster rate compared to least effective response selections. Although one might conclude that these simulation results support the proposition that most effective responding engages "shallow" reasoning and least effective responding engages "deeper" reasoning (cf., Brooks \& Highhouse, 2006), the response speed differences in SiRJ emerged as a function of the same single response process for both response choices. In this sense, SiRJ treats SJT responding in a manner more consistent with "unimodal" accounts of judgment (e.g., Kruglanski \& Gigerenzer, 2011) as opposed to dual process accounts that posit separable cognitive systems for intuitive and analytical reasoning (Evans, 2008).

The simulation results also produced larger average response speed differences between most and least effective selections in comparison to the observed empirical data; however, caution should be exercised in using the magnitude of these estimates as evidence against the validity of SiRJ. The response speed metrics analyzed in these two data sources are not directly comparable, which makes it difficult to determine precisely "how much" SiRJ differs from the empirical data or whether one response type is more systematically biased in the SiRJ model. Furthermore, certain parameters in SiRJ could be calibrated to more closely replicate the response speed differences observed in the empirical data if it were desirable (e.g., reduce magnitude of decision threshold for least effective response, increase magnitude of decision threshold for most effective response, etc.). Tuning these aspects of SiRJ could enhance its overall fidelity and capability to more precisely mimic observed response speed patterns, though decisions to do so should be based on more directed empirical research and justifiable theoretical rationale.

Of final note, the findings reported for $\mathrm{RQ3}$ also revealed that simulated respondents were much more likely to agree on what they perceived as the least effective versus the most effective response for an item, irrespective of whether a situational description was provided. This result was not anticipated and no existing predictions or evidence was found in the SJT literature to support this pattern. However, a post hoc rationale can be postulated that is consistent with these findings. For example, Stemler et al. (2016) posit that selecting the least effective response to an SJT item may reflect proscriptive knowledge regarding "what not to do" or what should be avoided in a situation. 
In comparison with prescriptive knowledge regarding "what to do" or what should be achieved in a situation, there is some evidence that proscriptive knowledge may be more commonly reflected in societal norms and laws and therefore more implicitly shared across individuals (i.e., "Thou shall not..." is more common than "Thou shall...", Anderson \& Dunning, 2014). SiRJ did not explicitly encode proscriptive or prescriptive knowledge in the experiential memory structures of simulated individuals. Instead, the most effective response was judged by the extent to which a particular response option was expected to produce more similar consequences to actions taken in the past, whereas the least effective response was judged by the extent to which a response option was likely to result in more dissimilar consequences. Consequently, the results produced by SiRJ are suggestive that knowledge of response features perceived as ineffective may be more commonly held across individuals. This does not mean that identifying the least effective response is necessarily "easier" for respondents; however, it does suggest that respondents may be more likely to agree on why a particular response is ineffective versus why a particular response is effective, even in the absence of explicit contextual information. These unanticipated results and preceding speculations suggest that continued reseach examining core differences between most and least effective responding would be valuable for further elaborating the nature of judgment and decision-making on SJT items and validating the general response process theory proposed in SiRJ.

\section{Practical Implications}

As with any new theory, advancing strong recommendations for practice should be contingent on further empirical validation of SiRJ. Nevertheless, correspondence between the simulation results and existing experimental data as well as support for the model's core process mechanisms in the broader decision sciences literature lends confidence to preliminary considerations of practical implications. First, the simulation results suggest that, although based on the same fundamental cognitive processes, most and least effective responding may reflect qualitatively different aspects of judgment. As a result, procedures that combine these responses into a single score may be missing out on useful information regarding respondents. For example, to the extent that most effective responding is characteristic of reasoning about the similarity between previously experienced consequences and anticipated consequences of response options, this response data may provide insights into more "heuristic-like" reasoning characteristic of a respondents' past experiences. By comparison, least effective response data may be telling of more "analytical-like" reasoning that conveys information about how a respondent reasons through potential actions they have never previously entertained. In any event, the SiRJ architecture implies that most and least effective 
responding likely hold different implications for the evaluation of SJT responding and thus may offer unique predictive capacities when analyzed separately (see also Elliott et al., 2011; Stemler et al., 2016).

Second, the neural network classification analyses performed on the simulation results demonstrated that differences in experiential knowledge (i.e., expertise type) were associated with distinguishable patterns of response option endorsement across SJT items. A similar logic and analytic approach could be applied to real SJT data to extract unique "judgment profiles" that could potentially be used to classify respondents into definable clusters. Although the present work utilized supervised neural networks, other classification methods (e.g., latent class analyses, support vector machines, decision trees, k-means clustering; Oswald \& Putka, 2015) could be pursued. Irrespective of the statistical technique, the process would entail using the full distribution of response option selections-as opposed to an aggregate total score based on agreement with expert opinion or an external job performance criterion - to identify and extract judgment profiles. Once developed for a given SJT, a likelihood of profile membership could be computed for any SJT respondent; furthermore, those profiles could be correlated with different outcome variables (e.g., job performance, organizational citizenship behaviors, counterproductive work behaviors) to establish their predictive validity. These approaches would require large datasets, but would share many of the same advantages of the cognitive diagnostic models for SJT scoring discussed by Sorrel and colleagues (2016) while removing the need to make subjective a priori judgments about the constructs believed to be associated with each SJT response option.

A third potential application of SiRJ is motivated by Lievens and Sackett's (2007) observation that we do not yet know what makes SJT items more or less "difficult." When SJTs are not comprised of items that contain an objectively correct response, evidentiary support for the most or least effective response is typically based on expert opinion rather than formal logic/principles (e.g., mathematics) or veridical knowledge/standards (e.g., medical diagnoses, standards of practice). As a result, the concept of item difficulty in SJTs is qualitatively different than that described in existing psychometric models (i.e., classical test theory, item response theory, etc.). However, the exemplar results from SiRJ shown in Figure 6 highlight ways in which SJT items are likely to be perceived as more difficult, such as when a test taker is faced with the dilemma of choosing the "better among all excellent" or "worst among all good" response. Although less commonly observed in the current simulations, choosing the "worst among all terrible" or "best among all bad" should be equally difficult to resolve.

These findings suggest that one potentially useful framework for conceptualizing difficulty in SJTs concerns the degree of uncertainty that respondents are faced with when responding to an item. ${ }^{9}$ Though there are many 
treatments of uncertainty in the organizational and decision sciences, Lipshitz and Strauss (1997) offer one framework that maps particularly well to SiRJ and SJTs in particular. In brief, these authors proposed that uncertainty can be attributed to three sources (decision alternatives, potential outcomes of decision alternatives, and the nature of the situation) and stem from three causes (incomplete information, inadequate understanding, and undifferentiated alternatives). These dimensions can be crossed to produce a taxonomy of uncertainty for judgment and decision applications. Table 8 adapts this taxonomy to the specific context of SJT items as well how these representations could be potentially operationalized in SiRJ. Each cell of this table offers a specific prediction regarding how the design of an SJT item could be altered to increase the uncertainty presented to respondents in an item and, by extension, its (perceived) difficulty. Of further interest, the SiRJ framework also highlights that these attributions and sources of uncertainty are not completely independent. For example, response alternatives can appear to be equally (un)desirable as a function of either (or both) incomplete information or inadequate understanding regarding the situational context presented in an SJT item stem.

The factors and proposed taxonomy summarized in Table 8 thus suggest a flexible and potentially robust method for scaling the perceived difficulty of SJT items. The computational model accompanying SiRJ can be used to explore and generate a priori predictions for how these different forms of uncertainty in isolation and combination might increase the perceived difficulty—as measured by differences in response speed or agreement/endorsement among response option selections_-of SJT items. The comparison of situation-absent and situation-present conditions in the present simulations offer a use case for applying this taxonomy. Removing the situational description from an SJT item reduces the information available to respondents to make a judgment (i.e., bottom-left cell of Table 8). As demonstrated by the simulation findings, the resultant broadening of the decision space introduced greater uncertainty and lower rates of agreement regarding the most and least effective response selections in this condition. However, adding uncertainty does not mean that respondents will necessarily be more likely to reach the "wrong" conclusion. As exemplified in Figures 4 and 5, similar response distributions were observed among test takers in both the simulated situation-present and situation-absent conditions. It simply means that items with less information are more likely to require additional cognitive effort to cope with the added uncertainty (Lipshitz \& Strauss, 1997), thereby increasing the apparent difficulty of an item.

Although it would require further refinement and empirical validation efforts, an additional future application of SiRJ could involve aiding decisions about the construction and composition of SJTs. Developers have pursued a variety of techniques for developing SJTs. For example, a critical incident approach may be used to elicit situations 
and/or responses from subject matter experts with some "top-down" consideration about the intended construct domains (e.g., "Tell me about a time when you behaved conscientiously at work"). Other times, the SJT designer may use more generic item stems with little focused attention to the nature of the stimulus situation, but then attempt to carefully construct response options to reflect more or less expression of a particular concept/construct (i.e., all items have responses with an "agreeable" option, an "avoidant" option, etc.). Still others may develop clusters of related item stems that appear related to broad domains of activity (conflict management, leadership, etc.) and then include response options believed to be more or less effective expressions of that activity irrespective of measuring a specific construct. This latter procedure is most consistent with the approach used to build the SJT in the reported simulations. However, each of these approaches represents a different logic to SJT construction that may have implications for the final composition of the assessment tool and subsequent interpretation of test taker responses. By directing additional attention to the process by which SJTs are constructed and the corresponding processes used by respondents when answering these items, the SiRJ model could serve as a tool for exploring the impact of different design, development, and scoring choices to the information derived from SJT measurement.

\section{Conclusion}

A common criticism of SJT research is that has been atheoretical and has eschewed potential explanatory accounts and research from the cognitive and decision sciences (Brooks \& Highhouse, 2006; Chan \& Schmitt, 2005; Foldes et al., 2010; Ployhart, 2006). A key driver of the present work was to begin to integrate the rich literature on judgment and decision-making into examinations of SJTs. Although applied specifically to SJTs, the proposed SiRJ framework represents a highly generalizable and flexible model of situated judgment and decision-making whose fundamental process mechanisms are rooted in basic psychological principles of memory and information processing. Consequently, this framework could potentially be adapted to fit other low-fidelity assessment techniques in the future (e.g., situational interviews, assessment center tasks; Weekley et al., 2015). The present simulations further demonstrated the capability of the generative process mechanisms in SiRJ to reproduce patterns consistent with empirical observations of SJT responding as well as generate new predictions and implications for future work in this area. Beyond its substantive contributions, SiRJ also offers a compelling demonstration of the potential for computational theories to achieve a more integrative science (Kozlowski et al., 2013; Wang et al., 2016; Vancouver \& Weinhardt, 2012). Concerted efforts to develop, test, and explore similar process-oriented theories and explanations for phenomena big and small reflect a tremendous opportunity to advance the rigor, precision, and explanatory potential of industrial and organizational psychology. 


\section{References}

Adolphs, R. (2002). Recognizing emotions from facial expressions: Psychological and neurological mechanisms. Behavioral and Cognitive Neuroscience Reviews, 1, 21-62.

Allaire, J.J., \& Chollet, F. (2019). keras: $R$ interface to 'Keras'. R package version 2.4.1.

Allaire, J.J., \& Tang ,Y. (2019). tensorflow: R interface to 'TensorFlow'. R package version 1.13.1.

Anderson, J.E., \& Dunning, D. (2014). Behavioral norms: Variants and their identification. Social and Personality Psychology Compass, 8, 721-738.

Baddely, A.D., \& Hitch, G. (1974). Working memory. In G.A. Bower (Ed.), The psychology of learning and motivation (Vol. 8, pp. 47-89). New York: Academic Press.

Brooks, M.E., \& Highhouse, S. (2006). Can good judgment be measured? In J.A. Weekley \& R.E. Ployhart (Eds.), Situational judgment tests: Theory, measurement, and application (pp. 39-55). Mahwah, NJ: Lawrence Erlbaum.

Busemeyer, J.R. \& Townsend, J.T. (1993). Decision field theory: A dynamic cognition approach to decision-making. Psychological Review, 100, 432-459.

Chan, D., \& Schmitt, N. (2005). Situational judgment tests. In A. Evers, N. Anderson, \& O. Voskuijil (Eds.), The Blackwell Handbook of Personnel Selection (pp. 219-242). Malden, MA: Blackwell Publishing.

Chase, W.G., \& Simon, H.A. (1973). Perception in chess. Cognitive Psychology, 4, 55-81.

Christian, M.S., Edwards, B.D, Bradley, J.C. (2010). Situational judgment tests: Constructs assessed and a metaanalysis of their criterion related validities. Personnel Psychology, 63, 83-117.

Coen, C. (2009). Simple but not simpler. Computational \& Mathematical Organization Theory, 15, 1-4.

Davis, J.P., Eisenhardt, K.M., \& Bingham, C.B. (2007). Developing theory through simulation methods. Academy of Management Review, 32, 480-499.

Denwood, M.J. (2016). runjags: An R package providing interface utilities, model templates, parallel computing methods and additional distributions for MCMC models in JAGS. Journal of Statistical Software, 71, 1-25.

Dougherty, M.R.P., Gettys, C.F., \& Ogden, E.E. (1999). MINERVA-DM: A memory processes model for judgments of likelihood. Psychological Review, 106, 180-209.

Dougherty, M.R.P., \& Hunger, J.E. (2003). Probability judgment and subadditivity: The role of working memory capacity and constraining retrieval. Memory \& Cognition, 31, 968-982.

Elliott, J.G., Stemler, S.E., Sternberg, R.J., Grigorenko, E.L., \& Hoffman, N. (2011). The socially skilled teacher and the development of tacit knowledge. British Educational Reseach Journal, 37, 83-103.

Engle, R.W., Tuholski, S.W., Laughlin, J.E., \& Conway, A.R.A. (1999). Working memory, short-term memory, and general fluid intelligence: A latent-variable approach. Journal of Experimental Psychology: General, 128, 309-331. 
Epstein, J. M. (1999). Agent-based computational models and generative social science. Complexity, 4, 41-60.

Evans, J.S.B.T. (2008). Dual-processing accounts of reasoning, judgment, and social cognition. Annual Review of Psychology, 59, 255-278.

Foldes, H., Ferro, G., Vasilopulous, N., Cullen, M., Wisecarver, M., \& Beal, S.A. (2010). Assessing judgment proficiency in army personnel (ARI Research Report\#1917). Arlington, VA: U.S. Army Research Institute for the Behavioral and Social Sciences.

Freeman, J.B., Penner, A.M., Saperstein, A., Scheutz, M., \& Ambady, N. (2011). Looking the part: Social status cues shape race perception. PLoS One, 6, e25107.

Gigerenzer, G., \& Hoffrage, U. (1995). How to improve Bayesian reasoning without instruction: Frequency formats. Psychological Review, 102, 684-704.

Gigerenzer, G., Hoffrage, U., \& Kleinbölting, H. (1991). Probabilistic mental models: A Brunswikian theory of confidence. Psychological Review, 98, 506-528.

Grand, J.A., Allen, M.T., \& Pearlman, K. (April, 2012). The role of general and specific knowledge in SJT scores. Symposium presented at the $27^{\text {th }}$ annual meeting of the Society for Industrial and Organizational Psychology, San Diego, CA.

Greenwald, A.G., Banaji, M.R., Rudman, L.A., Farnham, S.D., Nosek, B.A., \& Mellott, D.S. (2002). A unified theory of implicit attitudes, stereotypes, self-esteem, and self-concept. Psychological Review, 109, 3-25.

Guion, R.M. (1998). Assessment, measurement, and prediction for personnel decisions. Mahwah, NJ: Lawrence Erlbaum Associates.

Hammond, K.R. (1996). Human judgment and social policy: Irreducible uncertainty, inevitable error, unavoidable injustice. New York, NY: Oxford University Press.

Harris, A.M., Siedor, L.E., Fan, Y., Listyg, B., \& Carter, N.T. (2016). In defense of the situation: An interactionist explanation for performance on situational judgment tests. Perspectives on Science and Practice: Industrial and Organizational Psychology, 9, 23-28.

Harrison, J. R., Lin, Z., Carroll, G. R., \& Carley, K. M. (2007). Simulation modeling in organizational and management research. Academy of Management Review, 32, 1229-1245.

Hastie, R. (2001). Problems for judgment and decision-making. Annual Review of Psychology, 52, 653-683.

Judge, T.A., \& Zapata, C.P. (2015). The person-situation debate revisited: Effect of situation strength and trait activation on the validity of the Big Five personality traits in predicting job performance. Academy of Management Journal, 58, 1149-1179.

Kahneman, D. (2003). A perspective on judgment and choice: Mapping bounded rationality. American Psychologist, $58,697-720$.

Klein, G. (1997). The recognition-primed decision-model (RPD) model: Looking back, looking forward. In C.E. Zsambok \& G. Klein (Eds.), Naturalistic decision-making (pp. 285-292). Mahwah, NJ: Lawrence Erlbaum. 
Kozlowski, S.W.J., Chao, G.T., Grand, J.A., Braun, M.T., \& Kuljanin, G. (2013). Advancing multilevel research design: Capturing the dynamics of emergence. Organizational Research Methods, 16, 581-615.

Kruglanski, A., \& Gigerenzer, G. (2011). Intuitive and deliberate judgments are based on common principles. Psychological Review, 118, 97-109.

Kruglanski, A.W., Shah, J.Y., Fishbach, A., Friedman, R., Chun, W.Y., \& Sleeth-Keppler, D. (2002). A theory of goal systems. In M.P. Zanna (Ed.), Advances in experimental social psychology (Vol. 34, pp. 331-378). San Diego, CA: Academic Press.

Kruglanski, A.W., Thompson, E.P., Higgins, E.T., Atash, M.N., Pierro, A., Shah, J.Y., \& Spiegel, S. (2000). To "do the right thing" or to "just do it": Locomotion and assessment as distinct regulatory imperatives. Journal of Personality and Social Psychology, 79, 793-815.

Krumm, S., Lievens, F., Huffmeier, J., Lipnevich, A.A., Bendels, H., \& Hertel, G. (2015). How "situational" is judgment in situational judgment tests? Journal of Applied Psychology, 100, 399-416.

Kruschke, J.K. (2015). Doing Bayesian data analysis. (2 ${ }^{\text {nd }}$ ed.). Burlington, MA: Academic Press.

Kruschke, J.K., Aguinis, H., \& Joo, H. (2012). The time has come: Bayesian methods for data analysis in the organizational sciences. Organizational Research Methods, 15, 722-752.

Leeds, J.P. (2012). The theory of cognitive acuity: Extending psychophysics to the measurement of situational judgment. Journal of Neuroscience, Psychology, and Economics, 5, 166-181.

Lievens, F. (2006). International situational judgment tests. In J.A. Weekley \& R.E. Ployhart (Eds.), Situational judgment tests: Theory, measurement, and application (pp. 279-300). Mahwah, NJ: Lawrence Erlbaum.

Lievens, F., Buyse, T., \& Sackett, P.R. (2005). The operational validity of a video-based situational judgment test for medical college admissions: Illustrating the importance of matching predictor and criterion construct domains. Journal of Applied Psychology, 90, 442-452.

Lievens, F., \& Motowidlo, S. (2016). Situational judgment tests: From measures of situational judgment to measures of general domain knowledge. Perspectives on Science and Practice: Industrial and Organizational Psychology, 9, 3-22.

Lievens, F., Peeters, H., \& Schollaert, E. (2008). Situational judgment tests: A review of recent research. Personnel Review, 37, 426-441.

Lievens, F., \& Sackett, P.R. (2007). Situational judgment tests in high-stakes settings: Issues and strategies with generating alternate forms. Journal of Applied Psychology, 92, 1043-1055.

Lipshitz, R., Klein, G., Orasanu, J., \& Salas, E. (2001). Taking stock of naturalistic decision-making. Journal of Behavioral Decision-making, 14, 331-352.

Lipshitz, R., \& Strass. O. (1997). Coping with uncertainty: A naturalistic decision-making analysis. Organizational Behavior and Human Decision Processes, 69, 149-163.

Love, B.C., Medin, D.L., \& Gureckis, T.M. (2004). SUSTAIN: A network model of category learning. Psychological Review, 111, 309-332. 
Mayes, A. R., \& Montaldi, D. (2001). Exploring the neural bases of episodic and semantic memory: The role of structural and functional neuroimaging. Neuroscience \& Biobehavioral Reviews, 25, 555-573.

McDaniel, M.A., Morgeson, F.P., Finnegan, E.B., Campion, M.A., \& Braverman, E.P. (2001). Use of situational judgments tests to predict job performance: A clarification of the literature. Journal of Applied Psychology, $86,730-740$.

McDaniel, M.A., \& Nguyen, N.T. (2001). Situational judgment tests: A review of practice and constructs assessed. International Journal of Selection and Assessment, 9, 103-113.

McRae, K., \& Jones, M.N. (2013). Semantic memory. In D. Reisberg (Ed.), The Oxford Handbook of Cognitive Psychology (pp. 206-216). New York, NY: Oxford University Press.

Medin, D.L., Goldstone, R.L., \& Genter, D. (1993). Respects for similarity. Psychological Review, 100, 254-278.

Medin, D.L., \& Schaffer, M.M. (1978). Context theory of classification learning. Psychological Review, 85, 207-238.

Meyer, R.D., Dalal, R.S., \& Hermida, R. (2010). A review and synthesis of situational strength in the organizational sciences. Journal of Management, 36, 121-140.

Miller, J. H., \& Page, S. E. (2007). Complex adaptive systems. Princeton, NJ: Princeton University Press.

Mills, M. (2010). Introducing survival analysis and event history analysis. Thousand Oaks, CA: SAGE.

Miroshnikov, A., \& Conlon, E. (2014). parallelMCMCcombine: Methods for combining independent subset Markov Chain Monte Carol (MCMC) posterior samples to estimate a posterior density given the full dataset. $\mathrm{R}$ package version 1.0 .

Motowidlo, S.J., \& Beier, M.E. (2010). Differentiating specific job knowledge from implicit trait policies in procedural knowledge measured by a situational judgment test. Journal of Applied Psychology, 95, 321-333.

Motowidlo, S.J., Crook, A.E., Kell, H.J., \& Naemi, B. (2009). Measuring procedural knowledge more simply with a single-response situational judgment test. Journal of Business and Psychology, 24, 281-288.

Motowidlo, S.J., Dunnette, M.D., \& Carter, G.W. (1990). An alternative selection procedure: The low fidelity simulation. Journal of Applied Psychology, 75, 640-647.

Motowidlo, S.J., Hooper, A.C., \& Jackson, H.L. (2006a). A theoretical basis for situational judgment tests. In J.A. Weekley \& R.E. Ployhart (Eds.), Situational judgment tests: Theory, measurement, and application (pp. 5781). Mahwah, NJ: Lawrence Erlbaum.

Motowidlo, S.J., Hooper, A.C., \& Jackson, H.L. (2006b). Implicit policies about relations between personality traits and behavioral effectiveness in situational judgment items. Journal of Applied Psychology, 91, 749-761.

Motowidlo, S.J., \& Tippins, N. (1993). Further studies of the low-fidelity simulation in the form of a situational inventory. Journal of Occupational and Organizational Psychology, 66, 337-344.

Mumford, T.V., van Iddekinge, C.H., Morgeson, F.P., \& Campion, M.A. (2008). The team role test: Development and validation of a team role knowledge situational judgment test. Journal of Applied Psychology, 93, 250-267. 
Naylor, T.H., Finger, J.M., McKenney, J.L., Schrank, W.E., \& Holt, C. (1967). Verification of computer simulation models. Management Science, 14, B92-B106.

Nisbett, R.E., \& Wilson, T.D. (1977). Telling more than we can know: Verbal reports on mental processes. Psychological Review, 84, 231-259.

Nishii, L.H., Ployhart, R.E., Sacco, J.M., Wiechmann, D., \& Rogg, K.L. (2001, April). The influence of culture on situational judgment test responses. Paper presented at the $16^{\text {th }}$ Annual Conference of the Society for Industrial and Organizational Psychology, San Diego, CA

Nosofsky, R.M. (1986). Attention, similarity, and the identification-categorization relationship. Journal of Experimental Psychology: General, 115, 39-57.

Oppenheimer, D.M., \& Kelso, E. (2015). Information processing as a paradigm for decision making. Annual Review of Psychology, 66, 277-294.

Oswald, F.L., \& Putka, D.J. (2015). Statistical methods for Big Data: A scenic tour. In S. Tonidandel, E.B. King, \& J.M. Cortina (Eds.), Big Data at work: The data science revolution and organizational psychology (pp. 4363). New York, NY: Routledge.

Oswald, F.L., Schmitt, N., Kim, B.H., Ramsay, L.J., \& Gillespie, M.A. (2004). Developing a biodata measure and situational judgment inventory as predictors of college student performance. Journal of Applied Psychology, 89, 187-207.

Ployhart, R.E. (2006). The predictor response process model. In J.A. Weekley \& R.E. Ployhart (Eds.), Situational judgment tests: Theory, measurement, and application (pp. 83-105). Mahwah, NJ: Lawrence Erlbaum.

Ployhart, R.E. (2003). Be careful what you ask for: Effects of response instructions on the construct validity and reliability of situational judgment tests. International Journal of Selection and Assessment, 11, 1-16.

Plummer, M. (2003). JAGS: A program for analysis of Bayesian graphical models using Gibbs sampling. Proceedings of the 3rd International Workshop on Distributed Statistical Computing, 124, 1-8.

Phillips, J.K., Klein, G., \& Seik, W.R. (2004). Expertise in judgment and decision-making: A case for training intuitive decision skills. In D.J., Koehler \& N. Harvey (Eds.), Blackwell handbook of judgment and decision-making (pp. 297-315). Oxford, UK: Blackwell Publishing.

R Core Team. (2018). R: A language and environment for statistical computing. R Foundation for Statistical Computing, Vienna, Austria (www.R-project.org).

Rand, W., \& Rust, R.T. (2011). Agent-based modeling in marketing: Guidelines for rigor. International Journal of Research in Marketing, 28, 181-193.

Rockstuhl, T., Ang, S., Ng, K-Y., Lievens, F., \& Van Dyne, L. (2015). Putting judging situations into situational judgment tests: Evidence from intercultural multimedia SJTs. Journal of Applied Psychology, 100, 464-480.

Sargent, R.G. (2013). Verification and validation of simulation models. Journal of Simulation, 7, 12-24.

Schmidt, F.L., \& Hunter, J.E. (1993). Tacit knowledge, practical intelligence, general mental ability, and job knowledge. Current Directions in Psychological Science, 2, 8-9. 
Simon, H.A. (1956). Rational choice and the structure of the environment. Psychological Review, 63, 129-138.

Somers, J. (2018, April 5). The scientific paper is obsolete: Here's what's next. The Atlantic. Retrieved from https://www.theatlantic.com/science/archive/2018/04/the-scientific-paper-is-obsolete/556676/.

Sorrel, M.A., Olea, J., Abad, F.J., de la Torre, J., Aguado, D., \& Lievens, F. (2016). Validity and reliability of situational judgment test scores: A new approach based on cognitive diagnosis models. Organizational Research Methods, 19, 506-532.

SR Research EyeLink, (2009). EyeLink 1000 User Manual [computer software]. Mississauga, Ontario, Canada.

Stemler, S.E., Aggarwal, V., \& Nithyanand, S. (2016). Knowing what NOT to do is a critical job skill: Evidence from 10 different scoring methods. International Journal of Selection and Assessment, 24, 229-245.

Stemler, S.E., \& Sternberg, R.J. (2006). Using situational judgment tests to measure practical intelligence. In J.A. Weekley \& R.E. Ployhart (Eds.), Situational judgment tests: Theory, measurement, and application (pp. 107131). Mahwah, NJ: Lawrence Erlbaum.

Sternberg, R.J., Wagner, R.K., Williams, W.M., Horvath, J.A. (1995). Testing common sense. American Psychologist, $50,912-927$.

Tett, R.P., Simonet, D.V.,Walser, B., \& Brown, C. (2013). Trait activation theory: Applications, developments, and implications for person-workplace fit. In N.D. Christiansen \& R.P. Tett (Eds.), Handbook of personality at work (pp. 71-100). New York,NY: Routledge.

Thomas, R.P., Doughtery, M.R., Sprenger, A.M., \& Harbison, J.I. (2008). Diagnostic hypothesis generation and human judgment. Psychological Review, 115, 155-185.

Tourangeau, R., Rips, L.J., \& Rasinski, K. (2000). The psychology of survey response. Cambridge, UK: Cambridge University Press.

Tulving, E. (2002). Episodic memory: From mind to brain. Annual Review of Psychology, 53, 1-25.

Tversky, A. (1977). Features of similarity. Psychological Review, 84, 327-352.

Van Horn, R.L. (1971). Validation of simulation results. Management Science, 17, 247-258.

Vancouver, J.B., \& Weinhardt, J.M. (2012). Modeling the mind and the milieu: Computational modeling for micro-level organizational researchers. Organizational Research Methods, 15, 602-623.

Vancouver, J.B., Weinhardt, J.M., \& Schmidt, A.M. (2010). A formal, computational theory of multiple-goal pursuit: Integrating goal-choice and goal-striving processes. Journal of Applied Psychology, 95, 985-1008.

Wang, M., Zhou, L., \& Zhang, Z. (2016). Dynamic modeling. Annual Review of Organizational Psychology and Organizational Behavior, 3, 241-266.

Wilensky, U., \& Rand, W. (2015). An introduction to agent-based modeling: Modeling natural, social, and engineered complex systems with NetLogo. Cambridge, MA: The MIT Press.

Weber, E.U., \& Johnson, E.J. (2009). Mindful judgment and decision making. Annual Review of Psychology, 60, 5385. 
Weekley, J.A., Hawkes, B., Guenole, N., \& Ployhart, R.E. (2015). Low-fidelity simulations. Annual Review of Organizational Psychology and Organizational Behavior, 2, 295-322.

Weekley, J.A., \& Jones, C. (1999). Further studies of situational tests. Personnel Psychology, 52, 679-700.

Weekley, J.A., Ployhart, R.E. (2005). Situational judgment: Antecedents and relationships with performance. Human Performance, 18, 81-104.

Wilensky, U., \& Rand, W. (2015). An introduction to agent-based modeling: Modeling natural, social, and engineered complex systems with NetLogo. Cambridge, MA: MIT Press.

Zyphur, M.J., \& Oswald, F.L. (2015). Bayesian estimation and inference: A user's guide. Journal of Management, 41, 390-420. 
Table 1.

Summary of model verification and validation activities conducted with SiRJ computational model

\begin{tabular}{|c|c|c|c|}
\hline Focus & Activity & Description & Summary \\
\hline \multirow{3}{*}{ Verification } & Documentation & $\begin{array}{l}\text { Conceptual model and } \\
\text { implemented model are } \\
\text { adequately described and } \\
\text { documented }\end{array}$ & $\begin{array}{l}\text { - Described in paper and online application } \\
\text { (https://grandjam.shinyapps.io/siri) } \\
\text { - Model and simulation code commented and available for } \\
\text { download (https://osf.io/uwdfm/) }\end{array}$ \\
\hline & Programmatic testing & $\begin{array}{l}\text { Model code and execution } \\
\text { tested to ensure it does } \\
\text { what the programmer } \\
\text { intends (i.e., free from } \\
\text { bugs) }\end{array}$ & $\begin{array}{l}\text { - Unit testing - operations carried out in each step of } \\
\text { model were tested as developed } \\
\text { - Group walkthrough - reviewed model and code with } \\
\text { independent researchers/experts } \\
\text { - Debugging walkthroughs - complete code executed line- } \\
\text { by-line to ensure computations carried out as intended }\end{array}$ \\
\hline & $\begin{array}{l}\text { Test cases and } \\
\text { scenarios }\end{array}$ & $\begin{array}{l}\text { Model functions examined } \\
\text { to ensure they operate as } \\
\text { intended in the conceptual } \\
\text { model }\end{array}$ & $\begin{array}{l}\text { - Corner cases - verified expected model behavior using } \\
\text { extreme values (e.g., response selection highly } \\
\text { predictable with perfect encoding fidelity and when SJT } \\
\text { situations/responses are identical to situations and } \\
\text { responses stored in memory) } \\
\text { - Relative value testing - code tested with different } \\
\text { parameter values to ensure outputs changed reliably with } \\
\text { expected inputs (e.g., lower decision thresholds produce } \\
\text { quicker decisions) }\end{array}$ \\
\hline \multirow{4}{*}{ Validation } & Micro-face validation & $\begin{array}{l}\text { Core concepts \& elements } \\
\text { of model have face validity }\end{array}$ & $\begin{array}{l}\text { - Model components (e.g., memory structures, elements of } \\
\text { SJT items) drawn from and supported by psychological } \\
\text { theory, research, and application }\end{array}$ \\
\hline & Macro-face validation & $\begin{array}{l}\text { Core process mechanisms } \\
\text { and outcomes of model } \\
\text { have face validity }\end{array}$ & $\begin{array}{l}\text { - Model mechanisms (e.g., conditional reasoning, } \\
\text { preference accumulation, similarity judgments) derived } \\
\text { from and supported by existing research/theory } \\
\text { - Ordering of model phases (interpret item stem } \rightarrow \\
\text { interpret response options } \rightarrow \text { select response option) } \\
\text { corresponds with theorized and observed SJT } \\
\text { responding } \\
\text { - }{ }^{*} \text { Compared model behavior against alternative model } \\
\text { specification }\end{array}$ \\
\hline & $\begin{array}{l}\text { Empirical input } \\
\text { validation }\end{array}$ & $\begin{array}{l}\text { Input parameters have } \\
\text { correspondence to real } \\
\text { world data }\end{array}$ & $\begin{array}{l}\text { - Examples of operationalizing person/HyGene parameters } \\
\text { (e.g., threshold values, encoding fidelity) presented in } \\
\text { Thomas et al. (2008) } \\
\text { - Environmental parameters constructed such that many } \\
\text { methods could be used for operationalization (e.g., } \\
\text { critical incident reports to derive situational base rates, } \\
\text { pairwise ratings to determine situational similarity) } \\
\text { - Most parameters left scale-free (e.g., motivation, working } \\
\text { memory not tied to specific measures) to accommodate } \\
\text { future scaling and model calibration (e.g., } \\
\text { parameterization based on percentiles, effect sizes) }\end{array}$ \\
\hline & $\begin{array}{l}\text { Empirical output } \\
\text { validation }\end{array}$ & $\begin{array}{l}\text { Model output has } \\
\text { correspondence with real } \\
\text { world data }\end{array}$ & $\begin{array}{l}\text { * } \text { *tylized facts - model output patterns compared to } \\
\text { observed/proposed response patterns } \\
\text { - * Real world data - examined correspondence between } \\
\text { model output, published empirical effects, and new } \\
\text { empirical data }\end{array}$ \\
\hline
\end{tabular}

${ }^{*}$ Activity evaluated using simulation results presented in the manuscript text

Note. Verification and validation steps based on guidelines for rigor in computational modeling by Rand and Rust (2011) 
Table 2

Experimental design for empirical data collection

\begin{tabular}{cccc}
\hline \multicolumn{4}{c}{ Conditions } \\
\hline $\mathrm{A}$ & $\mathrm{B}$ & $\mathrm{C}$ & $\mathrm{D}$ \\
\hline 1-ME & 1-LE & $36-\mathrm{ME}$ & $36-\mathrm{LE}$ \\
2-LE & $2-\mathrm{ME}$ & $35-\mathrm{LE}$ & $35-\mathrm{ME}$ \\
$3-\mathrm{ME}$ & $3-\mathrm{LE}$ & $34-\mathrm{ME}$ & $34-\mathrm{LE}$ \\
$4-\mathrm{LE}$ & $4-\mathrm{ME}$ & $33-\mathrm{LE}$ & $33-\mathrm{ME}$ \\
$\ldots$ & $\ldots$ & $\ldots$ & $\ldots$ \\
$36-\mathrm{LE}$ & $36-\mathrm{ME}$ & $1-\mathrm{LE}$ & $1-\mathrm{ME}$ \\
\hline $\mathrm{n}=32$ & $\mathrm{n}=31$ & $\mathrm{n}=33$ & $\mathrm{n}=29$ \\
\hline
\end{tabular}

Note. ME = most effective. $\mathrm{LE}=$ least effective. Numbers refer to the same SJT item. The digit-letter strings summarize the response type participants in each condition were instructed to provide for each item (e.g., 1-ME = most effective response for SJT item 1; 33-LE = least effective response for SJT item 33). 
Table 3.

Confusion matrix for neural network predictions of simulated respondent experience strength

\begin{tabular}{|c|c|c|c|c|c|c|c|c|c|}
\hline \multirow{2}{*}{ True Level } & \multicolumn{5}{|c|}{ Predicted Level } & \multicolumn{4}{|c|}{ Mean Classification Probability } \\
\hline & 1 & 2 & 3 & 4 & None & 1 & 2 & 3 & 4 \\
\hline 1 & 4430 & 1593 & 225 & 10 & 3742 & $46 \%$ & $29 \%$ & $14 \%$ & $7 \%$ \\
\hline 2 & 0 & 0 & 0 & 0 & 10000 & $33 \%$ & $29 \%$ & $23 \%$ & $18 \%$ \\
\hline 3 & 0 & 0 & 0 & 0 & 10000 & $18 \%$ & $23 \%$ & $27 \%$ & $31 \%$ \\
\hline 4 & 45 & 212 & 1244 & 3814 & 4685 & $9 \%$ & $16 \%$ & $29 \%$ & $45 \%$ \\
\hline Precision & .99 & 0 & 0 & .99 & & & & & \\
\hline Recall & .71 & NA & NA & .72 & & & & & \\
\hline $\begin{array}{l}\text { Classification } \\
\text { rate }\end{array}$ & .63 & 0 & 0 & .53 & & & & & \\
\hline Accuracy & & & .71 & & & & & & \\
\hline
\end{tabular}

Note. $n=10000$ observations for each parameter level. Predicted level columns indicate number of times model classified an observation as belonging to a given categorical level with probability $\geq 50 \%$; "None" column indicates number of times model was unable to predict level membership. Mean classification probability columns indicate the model estimated classification probability for each level averaged across all observations. Precision, recall, and classification rate metrics are computed for each level ("one-vs-all"), whereas accuracy is computed for all model predictions.

Precision = \# of correct level predictions / sum of column

Recall = \# of correct level predictions / sum of row (excluding None)

Classification rate $=$ total number of level predictions $/$ total number of level observations

Accuracy = \# of correct model predictions / total number of model predictions 
Table 4.

Confusion matrix for neural network predictions of simulated respondent expertise type

\begin{tabular}{|c|c|c|c|c|c|c|c|c|c|c|c|}
\hline \multirow{2}{*}{ True Level } & \multicolumn{6}{|c|}{ Predicted Level } & \multicolumn{5}{|c|}{ Mean Classification Probability } \\
\hline & 1 & 2 & 3 & 4 & 5 & None & 1 & 2 & 3 & 4 & 5 \\
\hline 1 & 7999 & 0 & 0 & 0 & 0 & 1 & .99 & $<.001$ & $<.001$ & $<.001$ & $<.001$ \\
\hline 2 & 0 & 7990 & 0 & 0 & 0 & 10 & $<.01$ & .99 & $<.001$ & $<.001$ & $<.001$ \\
\hline 3 & 0 & 1 & 7989 & 2 & 0 & 8 & $<.001$ & .001 & .99 & .001 & $<.001$ \\
\hline 4 & 0 & 0 & 1 & 7978 & 1 & 20 & $<.001$ & $<.001$ & .001 & .99 & .001 \\
\hline 5 & 0 & 0 & 0 & 1 & 7994 & 5 & $<.001$ & $<.001$ & $<.001$ & .001 & .99 \\
\hline Precision & 1 & .99 & .99 & .99 & 1 & & & & & & \\
\hline Recall & 1 & 1 & .99 & .99 & .99 & & & & & & \\
\hline $\begin{array}{l}\text { Classification } \\
\text { rate }\end{array}$ & 1 & .99 & .99 & .99 & .99 & & & & & & \\
\hline Accuracy & & & & & & & & & & & \\
\hline
\end{tabular}

Note. $\mathrm{n}=8000$ observations for each parameter level. See Table 3 note for details on table calculations. 
Table 5.

Confusion matrix for neural network predictions of evaluation criterion used by simulated respondents

\begin{tabular}{|c|c|c|c|c|c|}
\hline \multirow[b]{2}{*}{ True Level } & \multicolumn{3}{|c|}{ Predicted Level } & \multicolumn{2}{|c|}{ Mean Classification Probability } \\
\hline & $\begin{array}{c}\text { Predicted } \\
\text { Consequences }\end{array}$ & $\begin{array}{c}\text { Response } \\
\text { Behavior } \\
\end{array}$ & None & $\begin{array}{c}\text { Predicted } \\
\text { Consequences }\end{array}$ & $\begin{array}{l}\text { Response } \\
\text { Behavior }\end{array}$ \\
\hline Predicted Consequences & 19994 & 5 & 1 & .99 & .001 \\
\hline Response Behavior & 5 & 19995 & 0 & .002 & .99 \\
\hline Precision & .99 & & & & \\
\hline Recall & .99 & & & & \\
\hline Classification rate & .99 & & & & \\
\hline Accuracy & .99 & & & & \\
\hline
\end{tabular}

Note. $\mathrm{n}=20000$ observations for each parameter level. Precision, recall, classification rate, and accuracy based on overall model predictions. See Table 3 note for additional details on table calculations. 
Table 6.

Confusion matrix for neural network predictions of SJT type completed by simulated respondents

\begin{tabular}{|c|c|c|c|c|c|}
\hline \multirow{2}{*}{ True Level } & \multicolumn{3}{|c|}{ Predicted Level } & \multicolumn{2}{|c|}{$\begin{array}{c}\text { Mean Classification } \\
\text { Probability }\end{array}$} \\
\hline & $\begin{array}{c}\text { Situation- } \\
\text { Absent }\end{array}$ & $\begin{array}{l}\text { Situation- } \\
\text { Present }\end{array}$ & None & $\begin{array}{l}\text { Situation- } \\
\text { Absent }\end{array}$ & $\begin{array}{l}\text { Situation- } \\
\text { Present }\end{array}$ \\
\hline Situation-Absent & 19264 & 736 & 0 & .94 & .06 \\
\hline Situation-Present & 650 & 19350 & 0 & .06 & .94 \\
\hline Precision & & & & & \\
\hline Recall & & & & & \\
\hline Classification rate & & & & & \\
\hline Accuracy & & & & & \\
\hline
\end{tabular}


Table 7

Posterior parameter estimates from hierarchical Bayesian survival models for empirical and simulated data

\begin{tabular}{lcccc}
\hline \multicolumn{1}{c}{ Variables } & $b$ & $\begin{array}{c}95 \% \text { Credibility } \\
\text { Interval }\end{array}$ & $\begin{array}{c}\% \text { Posterior } \\
\text { Above/Below Zero }\end{array}$ & Odds Ratio \\
\hline Empirical Data (DV = response time) & -12.1 & {$[-12.7,-11.6]$} & $100 \%$ below & $<.001$ \\
$\quad$ Intercept & -.027 & {$[-.029,-.026]$} & $100 \%$ below & .97 \\
$\quad$ Word Count & .019 & {$[.015, .024]$} & $100 \%$ above & 1.02 \\
$\quad$ Item Order & .175 & {$[.088, .258]$} & $100 \%$ above & 1.19 \\
$\quad$ Response Type & & & & \\
& & & & \\
Simulated Data (DV = number of iterations) & -4.48 & {$[-4.49,-4.46]$} & $100 \%$ above & .01 \\
$\quad$ Intercept & .094 & {$[.081, .106]$} & $100 \%$ above & 1.10 \\
$\quad$ Item Stem & 1.20 & {$[1.19,1.22]$} & $100 \%$ above & 3.32 \\
$\quad$ Evaluation Criterion & 1.81 & {$[1.80,1.83]$} & $100 \%$ above & 6.11 \\
$\quad$ Response Type &
\end{tabular}

Note. All coefficients $(b)$ are given in log-odds, reported in their original unstandardized units, and represent the estimate with the highest posterior probability. Response time variable from empirical data measured in seconds. Word Count variable was grand-mean centered prior to analysis. Item Order was coded such that first item answered by respond $=0$. Response Type: 0 = least effective selection, $1=$ most effective selection

Item Stem: 0 = situation-absent, 1 = situation-present

Evaluation criterion: $0=$ consequences, $1=$ behavioral action 
Table 8.

Hypothesized factors contributing to the difficulty of items on situational judgment tests

\begin{tabular}{|c|c|c|c|}
\hline \multirow{2}{*}{$\begin{array}{l}\text { Sources of } \\
\text { Uncertainty }\end{array}$} & \multicolumn{3}{|c|}{ Causes of Uncertainty } \\
\hline & Incomplete information & Inadequate understanding & Undifferentiated alternatives \\
\hline \multirow[t]{2}{*}{$\begin{array}{l}\text { Response } \\
\text { Alternatives }\end{array}$} & $\begin{array}{l}\text { Response options lack detail } \\
\text { about action }\end{array}$ & $\begin{array}{l}\text { Single response option has } \\
\text { multiple interpretations }\end{array}$ & $\begin{array}{l}\text { Response options are highly } \\
\text { similar }\end{array}$ \\
\hline & $\begin{array}{l}\text { SiRJ: Response options for an } \\
\text { item contain many unknown } \\
\text { features relative to responses } \\
\text { encoded in memory }\end{array}$ & $\begin{array}{l}\text { SiRJ: Same response associated } \\
\text { with different response contexts } \\
\text { in memory }\end{array}$ & $\begin{array}{l}\text { SiRJ: Response options for an } \\
\text { item all contain highly similar } \\
\text { features }\end{array}$ \\
\hline \multirow[t]{2}{*}{$\begin{array}{l}\text { Response } \\
\text { Outcomes }\end{array}$} & $\begin{array}{l}\text { Link between response option } \\
\text { and consequence is opaque }\end{array}$ & $\begin{array}{l}\text { Single response option has } \\
\text { multiple realistic/plausible } \\
\text { consequences }\end{array}$ & $\begin{array}{l}\text { Perceived consequences of } \\
\text { response options are highly } \\
\text { similar }\end{array}$ \\
\hline & $\begin{array}{l}\text { SiRJ: Responses and/or } \\
\text { response contexts stored in } \\
\text { memory have many unknown } \\
\text { features (poor encoding fidelity) }\end{array}$ & $\begin{array}{l}\text { SiRJ: Same response associated } \\
\text { with multiple different response } \\
\text { contexts in memory }\end{array}$ & $\begin{array}{l}\text { SiRJ: Response contexts for } \\
\text { different responses share many } \\
\text { features in memory }\end{array}$ \\
\hline \multirow[t]{2}{*}{ Situation } & $\begin{array}{l}\text { Situation description lacks } \\
\text { precise detail about who, what, } \\
\text { where, when, and why }\end{array}$ & $\begin{array}{l}\text { Situation has multiple } \\
\text { interpretations }\end{array}$ & (Not applicable) \\
\hline & $\begin{array}{l}\text { SiRJ: Situations for an item } \\
\text { contain many unknown features } \\
\text { relative to situations encoded in } \\
\text { memory }\end{array}$ & $\begin{array}{l}\text { SiRJ: Same situation associated } \\
\text { with different situation contexts in } \\
\text { memory }\end{array}$ & (Not applicable) \\
\hline
\end{tabular}


Figure 1. Model of Situated Reasoning and Judgment (SiRJ)

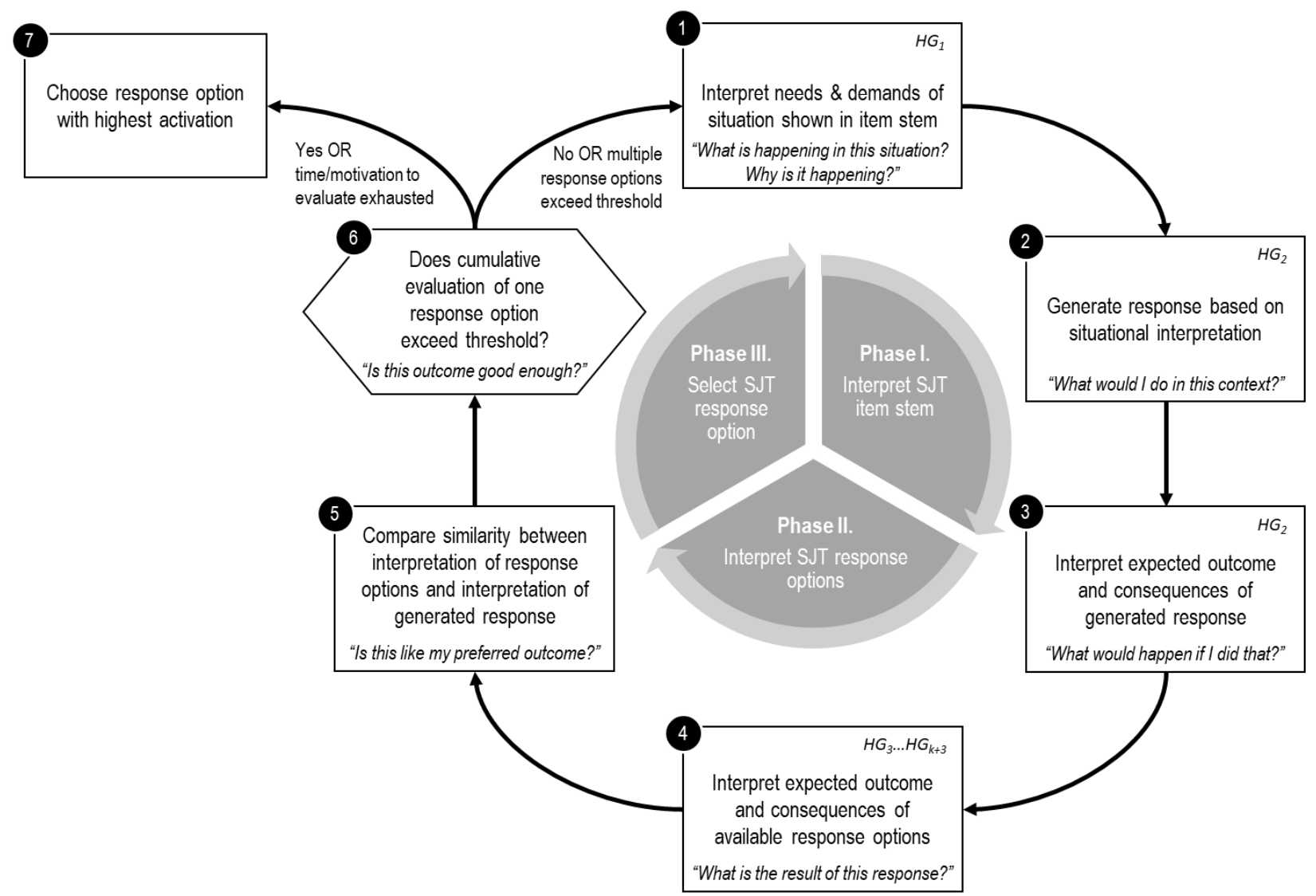

Note. The shaded circular diagram presents the three phases of SJT responding represented in SiRJ. The numbered white boxes summarize and indicate the order in which specific cognitive processing activities are carried out across these phases. The italicized text provides a heuristic description of the proposed cognitive appraisal that occurs during each stage. The $H G$ abbreviation indicate where unique instances of the conditional reasoning processes described in Thomas et al.'s (2008) HyGene architecture occur, with subscripts indicating the iteration of HyGene in which the associated evaluation is generated. $k=$ total number of available response options for an SJT item 
Figure 2. Distribution of most effective response selections for first ten simulated SJT items by experience strength (A) and expertise type (B) of simulated respondents

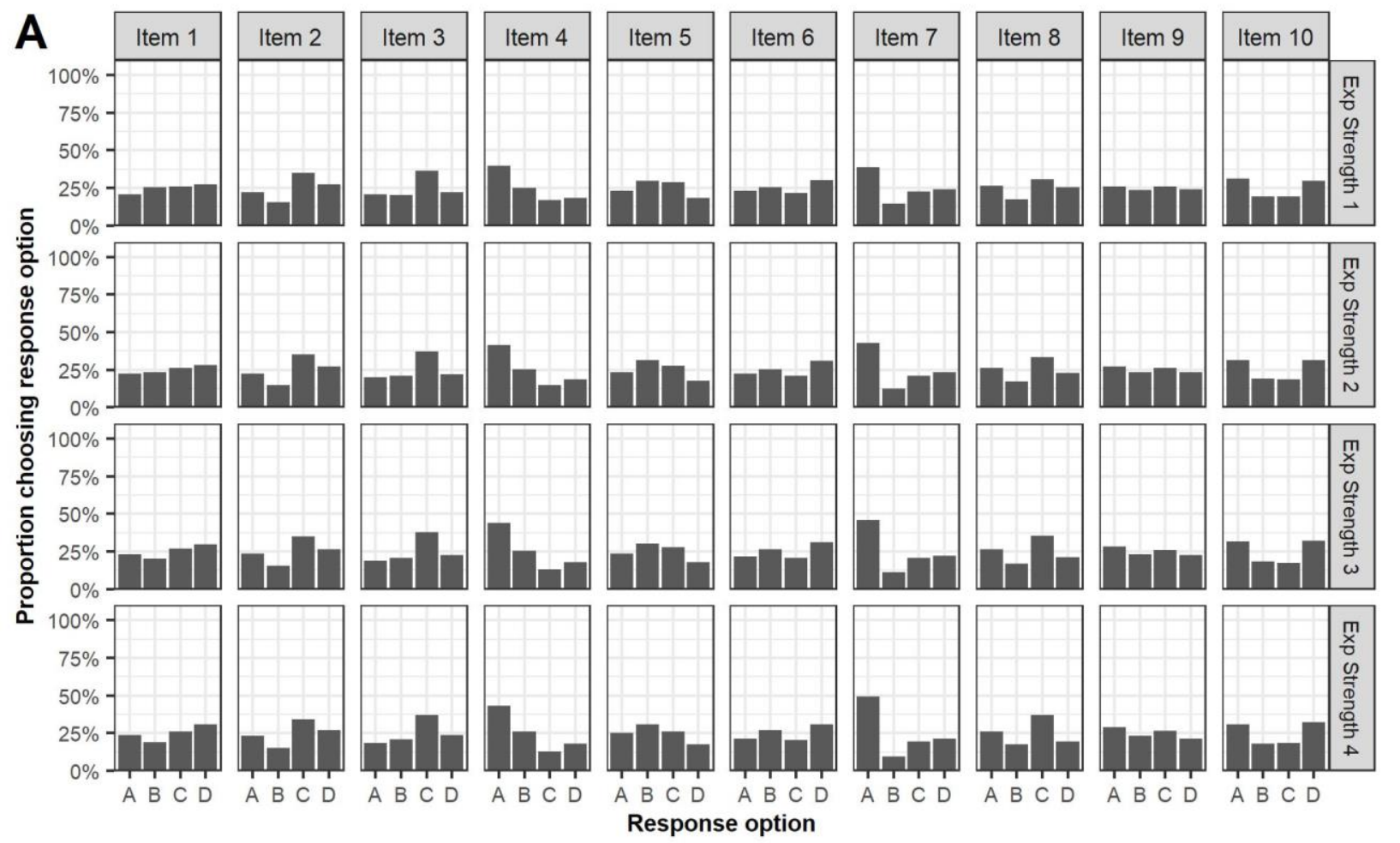

B
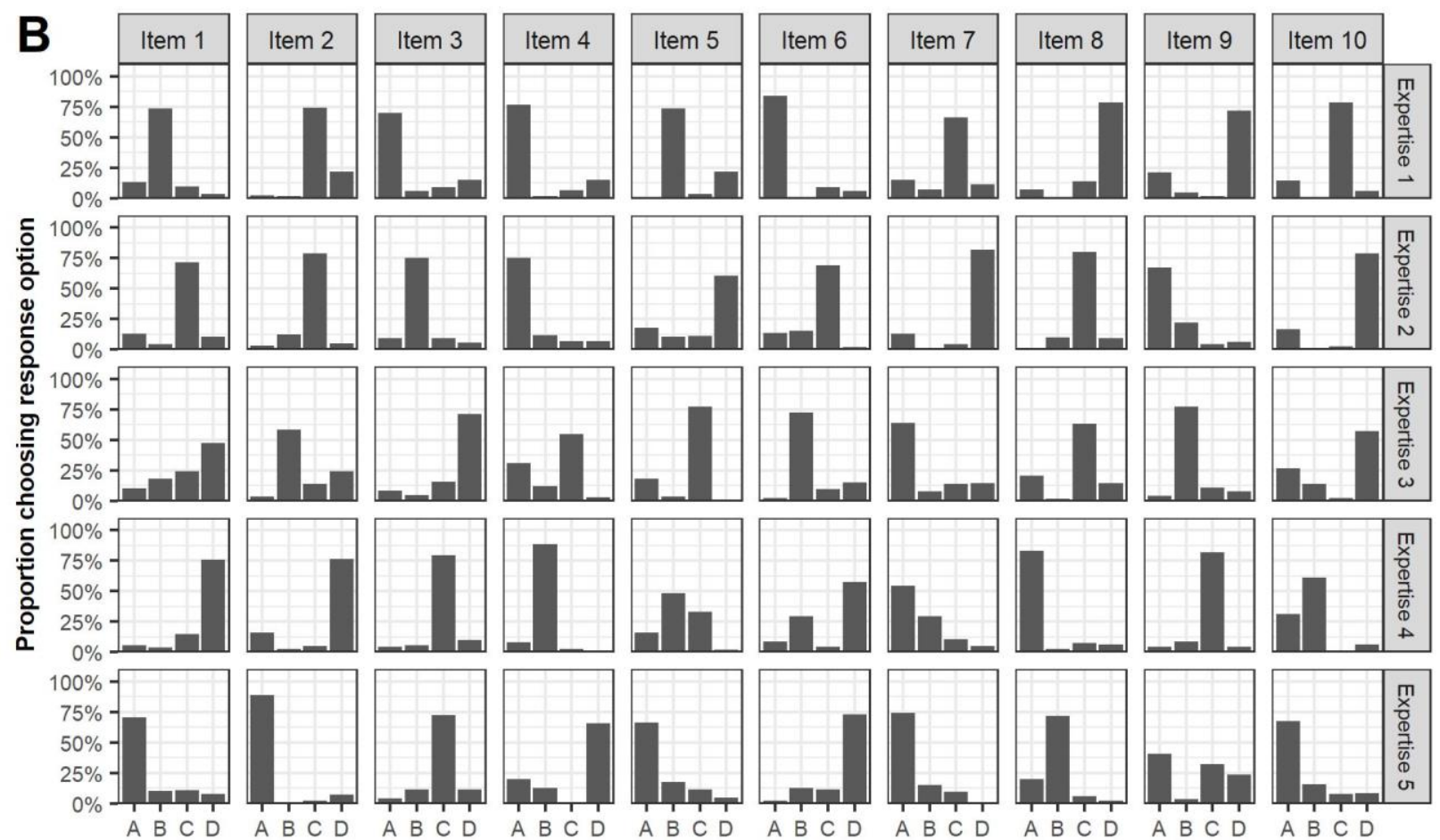

$A B C D \quad A B C D$ A B C D
Response option

A B C D

A B C D
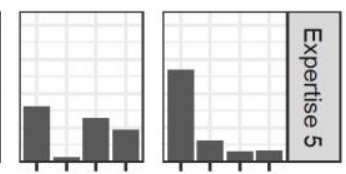
Figure 3. Distributions of most (A) and least (B) effective response selections by simulated respondents to SJT items by evaluation criterion used to interpret response effectiveness

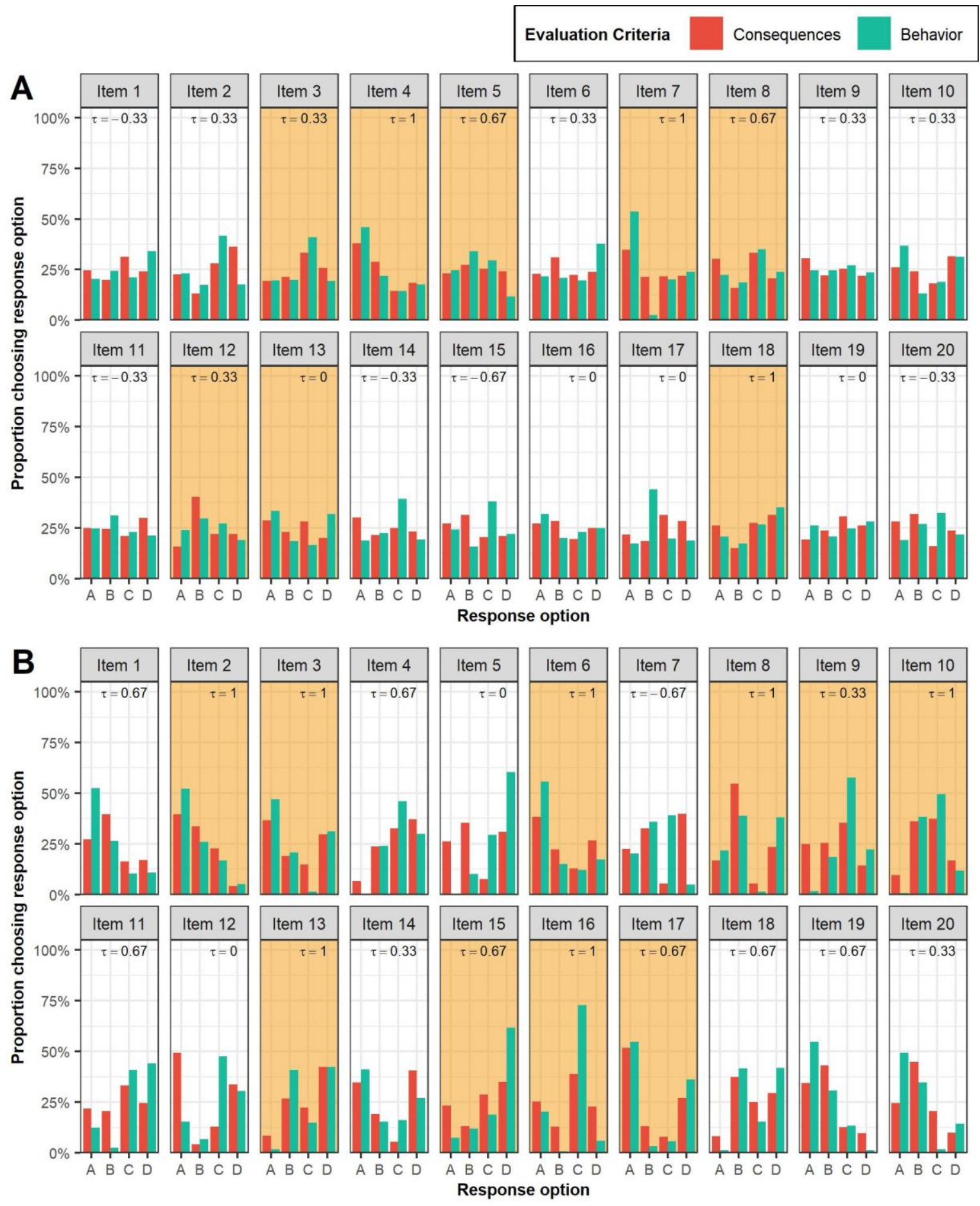

Note. $\tau=$ Kendall's tau indicating correlation between the rank ordering of response selection distributions (higher values $=$ more similar rank order). Highlighted cells indicate items for which the most frequently endorsed response option was the same for simulated respondents evaluating responses based on similarity of expected consequences (green bars) and those evaluating responses based on similarity of behavior (red bars). 
Figure 4. Distribution of most $(A)$ and least $(B)$ effective response selections by simulated respondents to SJT items by presence of situational description in item stems

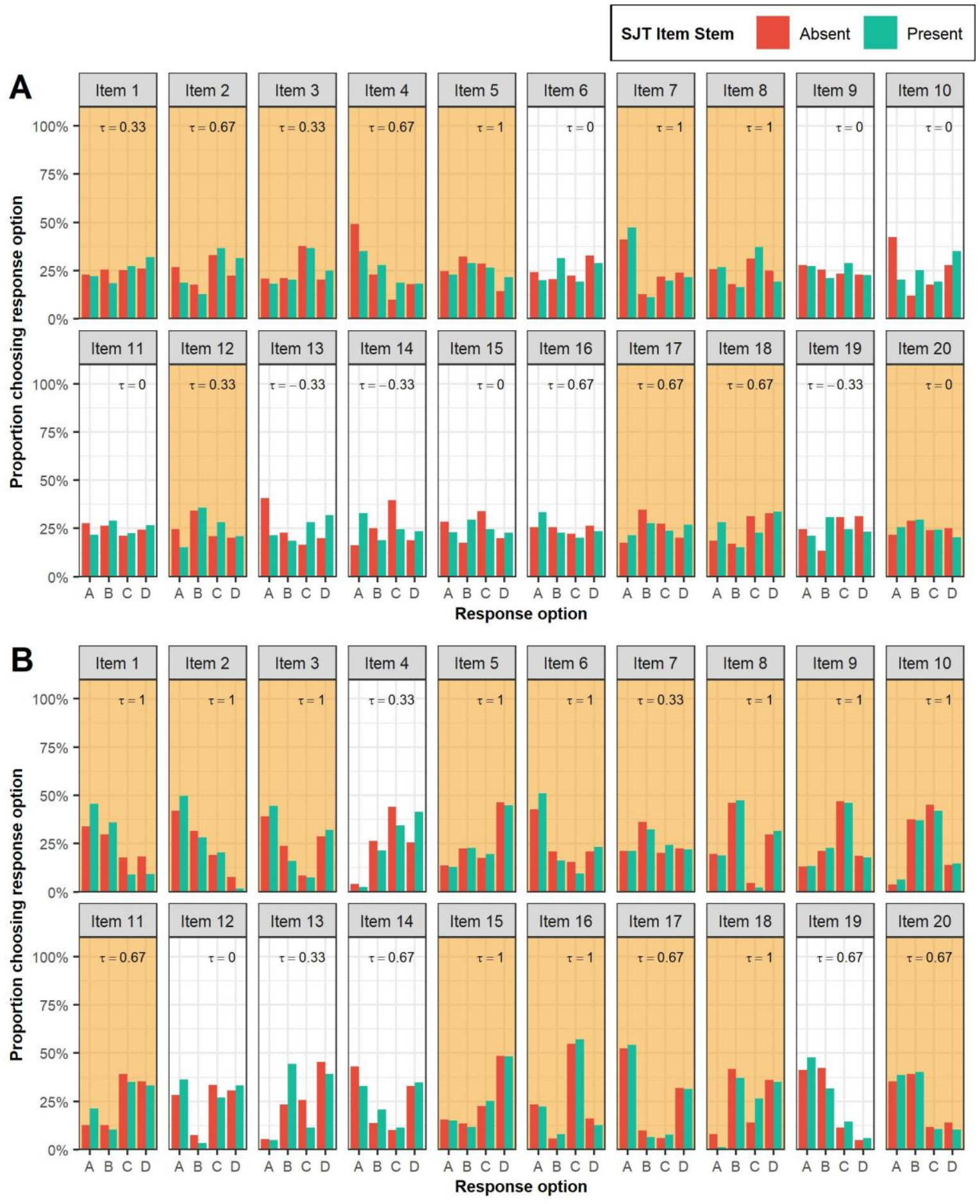

Note. $\tau=$ Kendall's tau indicating correlation between the rank ordering of response selection distributions (higher values $=$ more similar rank order). . Highlighted cells indicate items for which the most frequently endorsed response option was the same for simulated respondents taking the SJT with item stems (green bars) and those taking the SJT without item stems (red bars). 
Figure 5. Distribution of most effective response selections selected on first ten SJT items by expertise type and presence of situational description in item stems

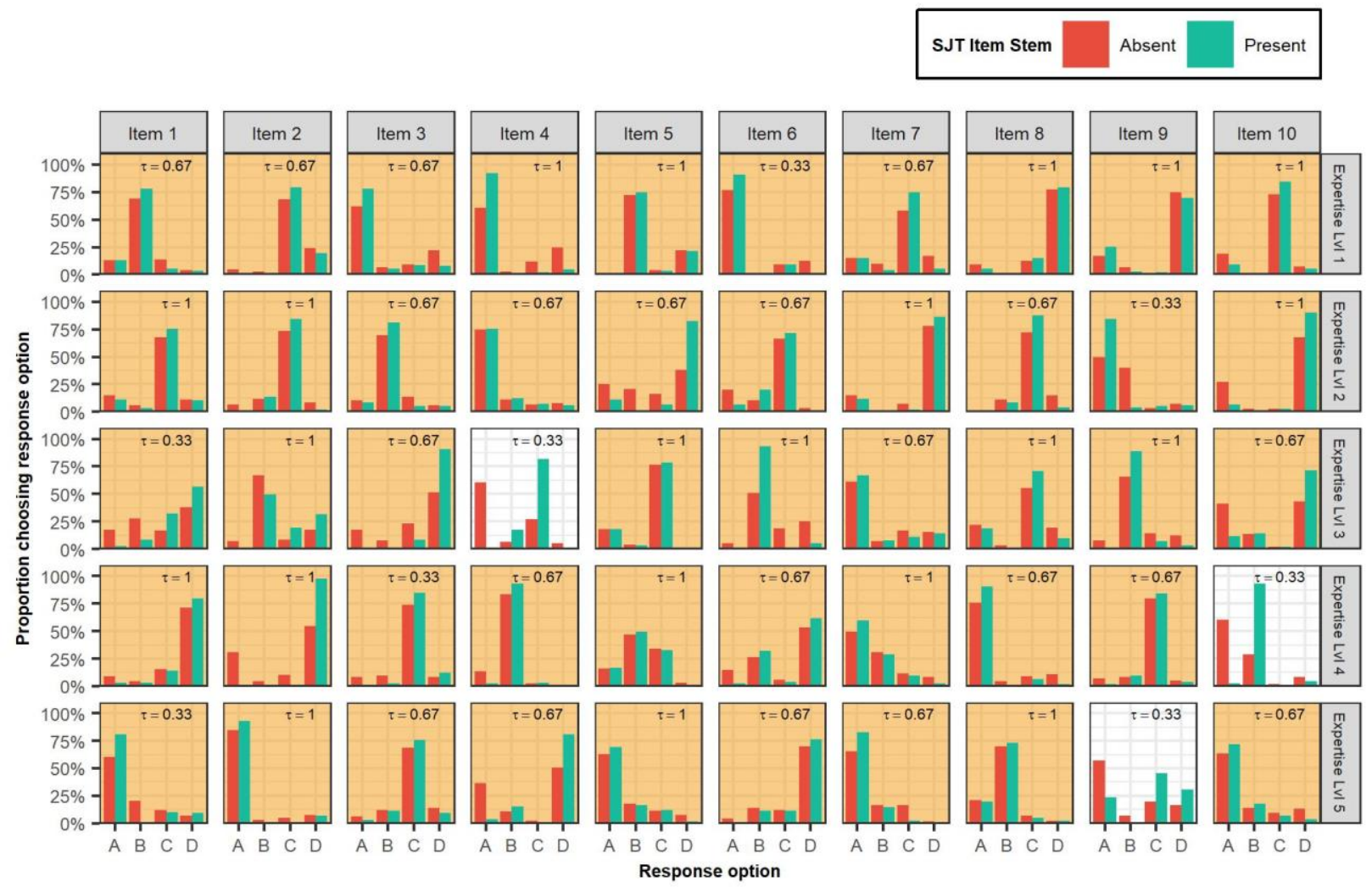

Note. $\tau=$ Kendall's tau indicating correlation between the rank ordering of response selection distributions (higher values $=$ more similar rank order). Highlighted cells indicate items for which the most frequently endorsed response option was the same for simulated respondents taking the SJT with item stems (green bars) and those taking the SJT without item stems (red bars). 
Figure 6. Example of response evaluation process carried out by single simulated respondent for five simulated SJT items
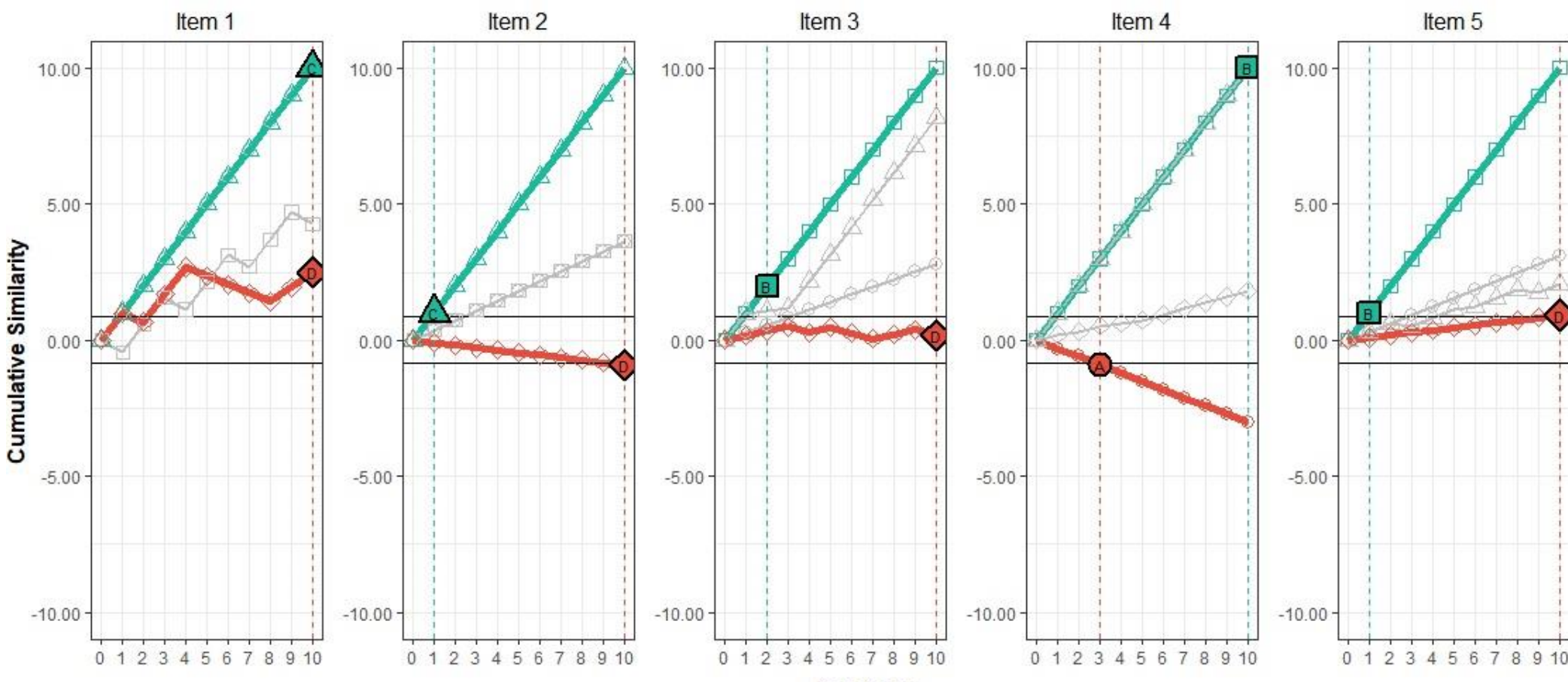

Response Type

ME

$-\mathrm{LE}$

Response

Option

$\mathrm{OA}$

$\square \mathrm{B}$

$\triangle \mathrm{C}$

Iterations

Note. Bolded green line/letter highlights the response option ultimately selected as most effective (ME) and bolded red line/letter highlights the response option ultimately selected as least effective (LE) for each item. Light gray lines depict the cumulative similarity judgments of response options not selected as either most or least effective. Horizontal black lines straddling zero on the $y$-axis reflect the thresholds for identifying the most (upper line) and least (lower line) effective response; these threshold values were held constant for all items and respondents (see Appendix B, Table B1). Vertical dashed lines highlight the iteration at which the simulated respondent identified the response options ultimately selected as most (green) and least (red) effective on each item. 
Figure 7. Survival plot of time to most and least effective response selection for simulated respondents averaged across all items by response type and item evaluation criterion

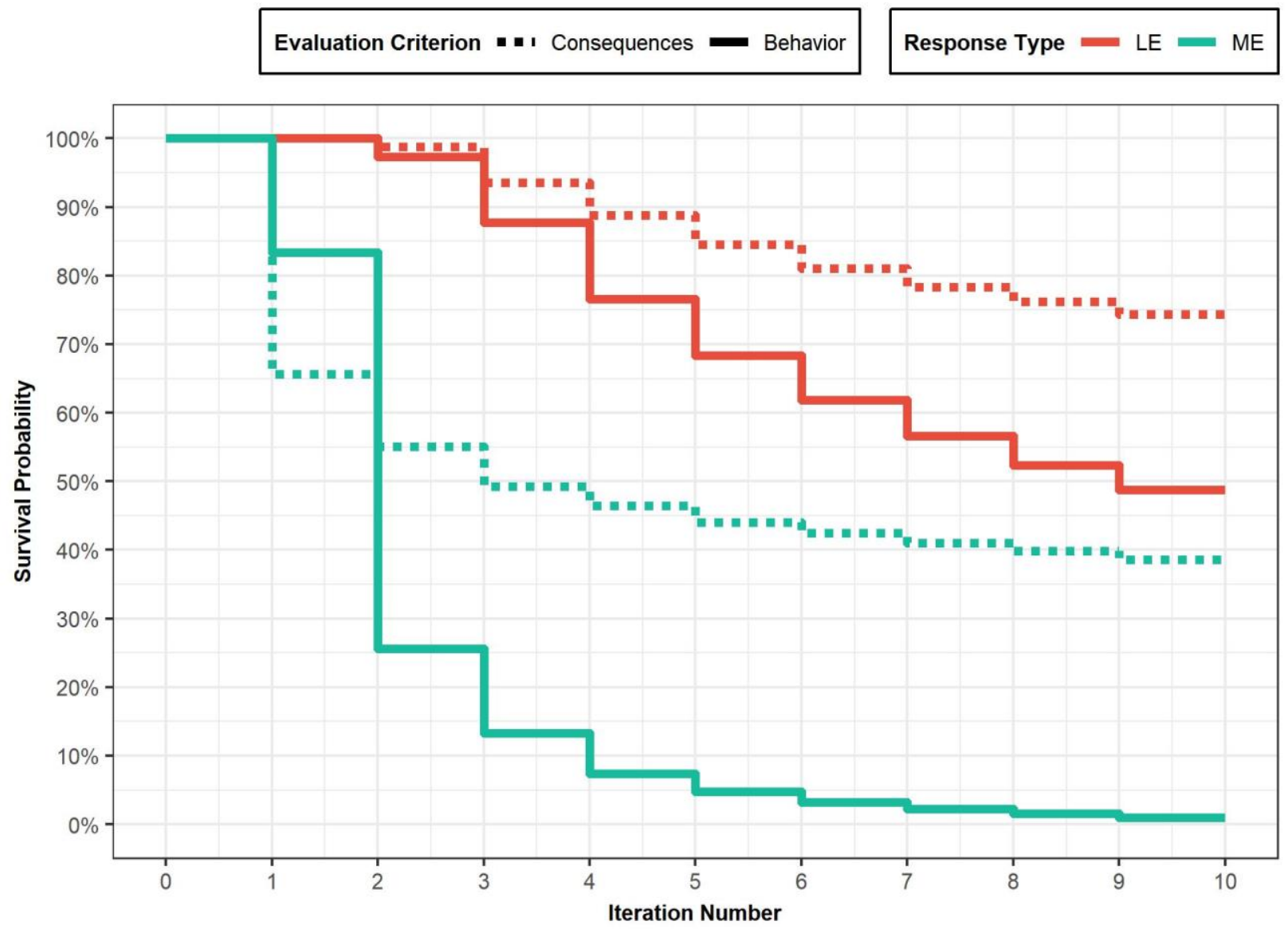

Note. Y-axis values indicate percentage of respondents who had not yet identified a most effective (ME, green line) or least effective (LE, red line) response by a particular iteration. X-axis values refer to the number of times through the item evaluation cycle shown in Figure 1 (e.g., Step 1-6). 


\section{APPENDIX A}

\section{Computational Model Description}

A detailed description of all formulas and computations involved in each step of $\mathrm{SiRJ}$ is provided at https://grandjam.shinyapps.io/siri. The material in this appendix is intended to provide a middle-range description of the core model operationalizations and process algorithms used in SiRJ.

\section{Model Operationalizations}

The foundations of SiRJ are built upon Thomas et al.'s (2008) HyGene model, which describes how conditional reasoning judgments can emerge from the interaction among three human memory systems-episodic memory, semantic memory, and working memory. The episodic memory system encompasses a person's "database" of past experiences and maintains a record of the base rates with which individuals have encountered particular events. Episodic memory stores (imperfect) representations of events—-known as traces in HyGene-and the interpretations associated with those experiences (Gigerenzer, Hoffrage, Kleinbölting, 1991; Tulving, 2002). For example, if an individual has a particularly hostile interaction with a supervisor, characteristics of the event (e.g., name of the supervisor, what the supervisor said, etc.) as well as associated inferences, responses, and outcomes of the event (e.g., why the supervisor was hostile, how the employee reacted, what happened when the employee reacted) are stored as a single trace in episodic memory. In contrast, the semantic memory system contains generalized representations from the episodic system as well as knowledge obtained outside of direct exposure. Semantic memory functions as storage for abstractions or prototypes of concepts derived from either direct (i.e., lived events) or indirect (i.e., "book" knowledge) experience (Mayes \& Montaldi, 2001; McRae \& Jones, 2013). An individual could thus maintain an overall appraisal of "a supervisor" in semantic memory; this representation would be informed by the employee's history of interactions with previous supervisors (e.g., episodic memory traces) as well as relevant knowledge learned through other means (e.g., personnel records, gossip, etc.). Lastly, working memory is neither a storage nor retrieval system, but rather a limited capacity "cognitive workspace" reflecting the extent to which individuals can maintain goal-relevant information in active awareness in the face of other demands (Baddely \& Hitch, 1974; Engle, Tuholski, Laughlin, \& Conway, 1999). Thus, if an individual was deciding whether to invite her supervisor to lunch, working memory would constrain how much information she could consider at any one point while making this decision.

Based on these definitions, HyGene posits that generating an explanation for an observed phenomena (e.g., "Why is my supervisor acting rudely?") is a Bayesian-like reasoning process in which a stimulus prompts a 
person to probe for relevant experiences in episodic memory, derive potential explanations from semantic memory, and then evaluate explanatory "contenders" in working memory to derive judgments about each explanation's validity. An episodic and semantic memory structure like that in HyGene is used to initialize the experiences, knowledge, and interpretations of respondents in SiRJ. Specifically, an episodic memory comprised of an associative network of traces representing a person's stored record of events is constructed for each simulated individual. Each trace is represented as a vector of features that may take the values $[1,0,-1]$. The features comprising a single trace are clustered into "minivectors" that correspond to an experienced event (situation), the individual's interpretation of the context surrounding that event (situation context), how the individual responded to the event (response), and the individual's interpretation of the consequences of that response (response context; see Panel A of Figure A1). The current representation of $\mathrm{SiRJ}$ is agnostic to the number or complexity of interpretations an individual associates with a situation or a response (e.g., the situation context and response context vectors). In this sense, an individual could be constructed to have a single complex interpretation of a situation/behavior stored in memory or a large number of simple interpretations encoded. A semantic memory system reflecting a person's learned prototypes is constructed by averaging the features of episodic memory traces pertaining to similar situations into singular prototype traces. Thus, semantic memory is operationalized as a series of traces reflecting an individual's prototypic perceptions relevant to a given "type" of situation (e.g., all conflict situations) and the situation context, response, and response context interpretations the individual associates with that type of event.

A similar format was used to construct SJT items in the SiRJ simulations. Specifically, each simulated SJT item contained an item stem and $k$ response options. SJT item stems were represented as a minivector of features consistent with the situational traces stored in simulated respondents' memory; similarly, each response option was represented as a minivector of features consistent with the response traces stored in respondents' memory. In this sense, situations that refer to a particular event in a simulated SJT item typically contained a combination of features similar to corresponding situations encoded in the episodic memory of simulated respondents; the same was true for

the correspondence between SJT response options and response traces in memory. Panel B of Figure A1 provides a visual representation of this operationalization.

\section{Model Procedures}

The overall item evaluation process represented in SiRJ is visually summarized in Figure 1 of the manuscript text and encompasses seven distinct steps. Steps 1-4 of this process entail a sequence of conditional reasoning judgments. Each of these steps occurs as a separate iteration of the judgment process described in 
Thomas et al.'s (2008) HyGene algorithm. For example, Step 1 in SiRJ proposes that the presentation of an SJT item stem prompts individuals to identify "Why is this situation happening?" (i.e., what is the situational context given the situation?). This reasoning process is carried out using the following procedures:

A. Features of the situation presented in the SJT item are recognized and formed into a "trace probe" (i.e., a search cue). This trace probe is compared against other situation traces stored in an individual's episodic memory to identify previously experienced situations that are similar. Although this stage appears to imply that individuals exert deliberate and conscious effort to comb through episodic memory each time they are faced with a stimulus, this need not be interpreted so literally. The functional outcome of this processing stage is the formulation of an overall impression of the trace probe and its associated information weighted by how frequently it has been experienced by an individual (i.e., its base-rate). More generally then, this stage represents how individuals interpret a stimulus through the lens of their own experiences in a way that can subsequently be ascribed meaning according to one's stored knowledge/prototypes.

B. An overall impression or "gist" of the situation and situation context reflected in the item stem is formulated by combining the features of any situations and situation contexts stored in episodic memory perceived as similar to the stimulus encoded in the trace probe.

C. The gist constructed from episodic memory is compared against the situation and situation context portion of traces stored in semantic memory to identify the extent to which an individual's overall impression matches any learned prototypes. In other words, the meaning of the just-formulated impression is deciphered by identifying how closely it aligns with previously stored and understood prototypes.

D. The degree of similarity between the gist and different semantic prototypes establishes the likelihood that any particular prototype will be considered among the "set of leading contenders" for the stimulus trace probe. The number of contenders that can be simultaneously considered is determined by the size of the individual's working memory.

E. Lastly, the probability that any contending interpretation identified from semantic memory offers an adequate explanation of the trace probe is determined based on its relative "memory strength" (i.e., how strongly a contending interpretation matches the interpretation of situations relevant to the trace probe stored in episodic memory). The SiRJ architecture assumes that individuals select the interpretation (in this example, the situation context) with the highest probability of explaining the trace probe as their preferred conclusion. 
The process outlined above is performed iteratively in Steps 1-4 of SiRJ such that the outcome of each step is used as input for subsequent stages. Specifically, the interpretation of the situation context extracted during Step 1 of SiRJ using the HyGene algorithm becomes the trace probe for eliciting an individual's typical response (i.e., generated response given situation context, Step 2) and interpretation of the consequences of that response (i.e., generated response context given response + situation context, Step 3) in a separate application of the HyGene algorithm. The extracted situation context also makes up part of the trace probe in Step 4 of SiRJ, which applies the HyGene procedure to each available response option to describe how individuals interpret the expected outcomes of the potential response options (i.e., response option context given response option + situation context).

The remaining Steps 5-7 of SiRJ describe how individuals utilize these evaluations to accumulate preferences for and against particular response options. This process is proposed to rely on similarity judgments made between the expected outcomes of the available response options (SiRJ Step 4) and the expected outcome of the simulated individual's generated response (SiRJ Step 3). The degree of similarity between interpretations in SiRJ is computed as their cosine similarity:

$$
\text { Cosine Similarity }=\frac{\sum_{i=1}^{n} A_{i} B_{i}}{\sqrt{\sum_{i=1}^{n} A_{i}^{2}} \sqrt{\sum_{i=1}^{n} B_{i}^{2}}}
$$

where $A_{i}$ and $B_{i}$ refer to the ith feature of vectors $A$ (i.e., response context generated in Step 3 of SiRJ) and $B$ (e.g., response context of Response Option A from Step 4 of SiRJ) and $n$ is the total number of features comprising the vectors. Values for cosine similarity are interpreted like a correlation coefficient and lie in the continuous range of [-1, 1]. A value of -1 means two vectors share complete opposite features, a value of +1 means they share identical features, and a value of 0 means they are orthogonal to one another (i.e., have just as many identical as opposite features). An SJT response option receiving a large positive cosine similarity during Step 5 of SiRJ thus indicates that a simulated individual perceives the expected outcomes of that response option to be highly similar to the anticipated outcome of the response the individual has most commonly associated with the perceived situation context. An SJT response option receiving a large negative cosine similarity indicates its anticipated outcome is highly dissimilar to the anticipated outcome of an individual's typical response.

In Step 6 of SiRJ, the cosine similarity computed for each response option is compared against a decision threshold to determine whether a most and least effective response option has been identified. Given the interpretation of the directionality and magnitude of the cosine similarity computation and the assumption that 
response options perceived to be more similar to an individual's typical/preferred pattern of responding are seen as more desirable (Motowidlo et al., 2006a; 2006b), a threshold with a positive value is used as the decision criterion for signifying the most effective response and a negative threshold for the least effective response. The magnitude of the thresholds represent the strength of evidence that must be achieved by a simulated individual to designate a particular response option as the most or least effective (e.g., larger positive [negative] values indicate that the simulated individual requires more evidence about a response before they will endorse it as the most [least] effective response to the presented situation). Note that simulated individuals make determinations about the effectiveness of response options based on the expected outcomes of those responses given their interpretation of the situation rather than the actual behavior reflected in a response. This operationalization affords the potential for different response options to be perceived as equally effective if the individual expects the consequences of different response options to be equivalent given the perceived situational context.

If one (and only one) response option exceeds the most effective threshold and one (and only one) response option exceeds the least effective threshold, those options are selected as such and the item evaluation process concludes (Step 7). If no options exceed either threshold, multiple options exceed either threshold, or either a most or least effective response option but not both has been identified, the item evaluation process continues (i.e., return to Step 1). After each deliberation, the similarity judgments attributed to a given response option are summed together. This accumulative process allows response options initially not seen as similar (or dissimilar) to a simulated individual's preferred response outcome to eventually be endorsed if the item evaluation process continues long enough. However, individuals are assumed to possess a limit on the amount of time they will continue to evaluate an item. This limit can be influenced by both intrinsic (e.g., motivation) or extrinsic (e.g., a time pressure) factors. If the evaluation limit is reached without a clear response choice, the simulated individual will either select the option that comes closest to exceeding a threshold (if no responses exceed a threshold) and/or randomly select a response (if multiple responses are perceived as being equally effective), and the evaluation process for that item terminates. 
Figure A1. Representation of memory traces (A) and SJT items (B) in SiRJ

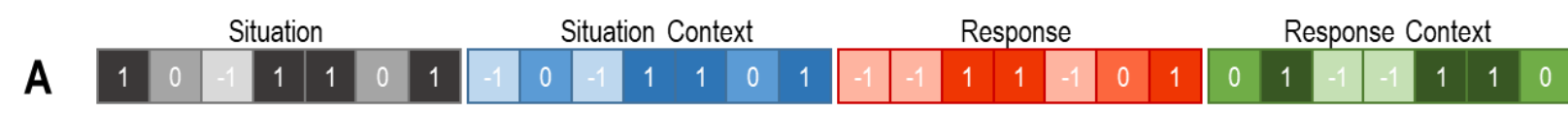

B
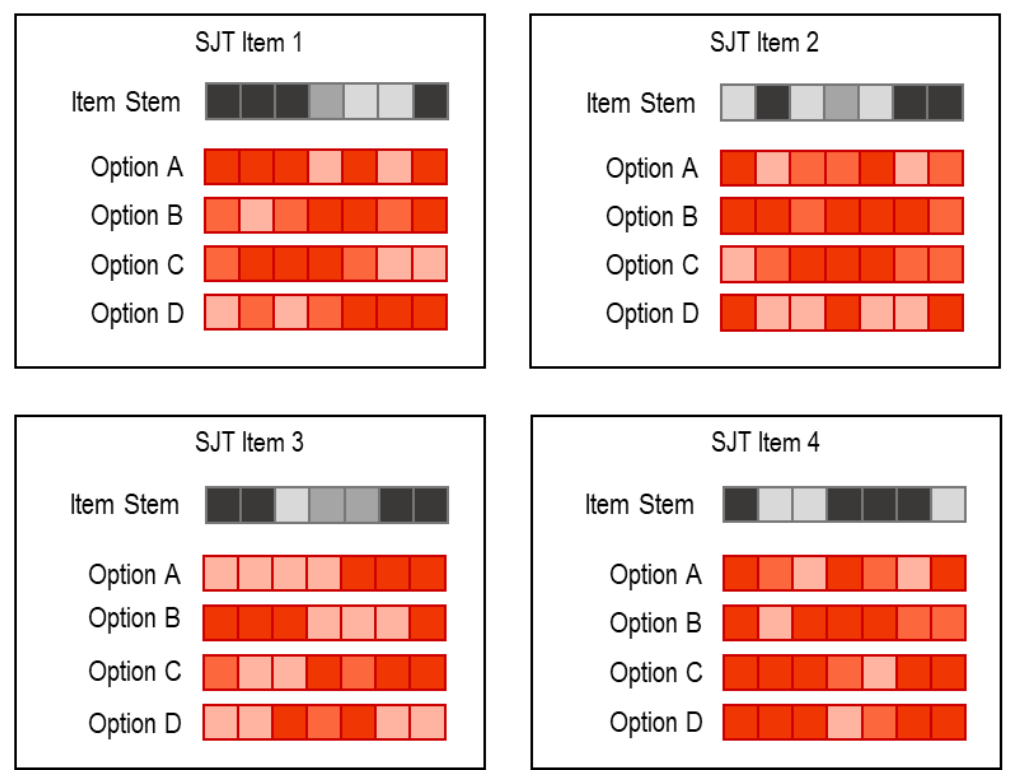

Note. Panel A provides an example of how a single memory trace is represented in SiRJ. Each cell in the situation, situation context, response, and response context portions of the trace represents a feature that could be present (1), absent (-1), or unknown (0) in the stored memory trace. The interactive web application at https://grandjam.shinyapps.io/sirj provides a tool for visualizing how this operationalization is used to represent a simulated individual's episodic and semantic memory systems in SiRJ. Panel B provides an example of how SJT items are represented in SiRJ. Item stems are operationalized as situations with a particular combination of features, whereas response options are operationalized as responses with a particular combination of features. 


\section{APPENDIX B}

\section{Parameterization of Computational Model and Simulations}

A goal of the SiRJ architecture was to develop a flexible and generalizable theory of situated reasoning and judgment that could be applied to SJT responding. Consequently, a variety of parameters were included in the baseline computational model for SiRJ that can be used to initialize key characteristics of the (1) person, (2) environment, and (3) SJT. Table B1 presents the parameters for each of these domains currently defined in the SiRJ model. The parameter values used in the reported simulation are also reported and discussed below.

The person parameters control characteristics of simulated respondents. The expertise type and experience strength parameters were the focal person-level manipulations in the simulations and determined the composition and size (respectively) of an individual's episodic memory. The experience strength manipulation resulted in four distinct clusters of episodic memory size for respondents (Cluster 1: mean $=315$ traces, $S D=9.9$; Cluster 2: mean $=$ $629, S D=13.9 ;$ Cluster 3: mean $=1258, S D=19.5$; Cluster 4: mean $=2516, S D=27.3$ ). The remaining person parameters were held constant. Values for these parameters were chosen to reflect individuals with accurate encoded memories $(L)$ and high evidentiary thresholds for both recognizing episodic memory traces based on trace probes $\left(A_{c}\right)$ and choosing a most and least effective response (MESim, LESim). Individuals were also afforded a moderate degree of motivation to search semantic memory (TMax) and continue deliberating over SJT items if no responses were unequivocally perceived as most or least effective (sjtMax). Lastly, individuals were made to be consistent in their reactions to particular situational interpretations $(S)$. In sum, the simulated test takers could be described as reasonably motivated individuals who possessed accurate memories of their lived experiences, moderately strong evidentiary decision standards, and tended to behave in a highly consistent/predictable manner over time (e.g., have strong implicit beliefs, Motowidlo et al., 2006a).

The next set of parameters in SiRJ control the composition of the simulated SJT. These include the number of items (numSjtltems) and response options per item stem (numSjtRsp) on the assessment. Two additional parameters were constructed to control the degree to which the situational features depicted in an item stem mirrored those found in the situational ecology (sjtSitSim) and the degree to which the features of a given response option mirrored those of a particular expertise type (sjtRspSim). Values for both parameters were selected so as to make situations and responses similar to the situational ecology and the responses most typical of a particular expertise type (respectively). 
The environment parameters in SiRJ do not control the nature of the test taking environment (e.g., high vs. low time pressure, high vs. low stakes testing, group vs. individual tests), but rather the situational ecology in which test takers are presumed to have "lived" and thus possess memories about. More specifically, these parameters are used to control the number of relevant situations in an environment (numSitType, numSpecSit), similarity among situations (simSitType, simSpecSit), the rarity of situations (brSitVar, sitTypeProb), and the degree of complexity with which the simulation designer wishes to operationalize potential situations, interpretations, and responses (MVLength, numSitMV, numSitCxtMV, numRspMV, numRspCxtMV).

The two most important aspects of setting the environmental ecology concern establishing the rarity and similarity of situations as these dictate the likelihood that individuals possess encoded memories of particular events and the degree of confusability among those events. The rarity of situations in SiRJ is determined by constructing probability distributions over broad classes of situations (e.g., conflict) that are subsequently used to sample the probability that more specific situations within that class (e.g., conflict with coworker, conflict with boss, conflict with customer) would be experienced. The interactive web application (https://grandjam.shinyapps.io/siri) offers more specific details and a hands-on tool demonstrating how this operationalization functions. For the present simulations, parameter values for situation rarity were selected such that the 15 specific situations modeled were fairly common; thus, all individuals possessed at least some experiences relevant to every situation included in the SJT. Figure B1 provides a visualization of how the situations simulated individuals could experience were constructed and is similar to that used by Thomas et al. (2008) in their original simulation experiments with the HyGene model. In brief, a single situation trace is constructed by randomly selecting values of -1 and 1 for all its features. A set of broad situation class traces are then constructed from this random trace by changing each of its features with probability = (1$\left.\operatorname{Sim}_{b s}\right)$. Finally, a set of specific situations are constructed from each of the broad situation class traces by changing each of their features with probability $=\left(1-\operatorname{Sim}_{s s}\right)$. For the present simulations, parameter values for situation similarity were selected such that broad situation classes were relatively distinct from one another, but specific situations within each broad situation class were fairly similar.

\section{Model Extensions}

The Discussion section of the main manuscript text describes several directions in which the present computational model for SiRJ could be extended. In addition to those conceptual targets, there are a number of parameters and functional relationships that could be built upon the parameters listed in Table B1 to introduce and/or represent more complex relationships of interest. In general, there are important tradeoffs when adding new 
complexities to a computational model (Coen, 2009); however, considering how and where to build upon or integrate new ideas into a theoretical model can often prove a useful exercise for refining and improving computational theory.

For example, elements related to motivation and/or characteristics of the testing environment could be introduced into SiRJ through both the Tmax and sjtMax parameters. Tmax is a parameter of Thomas et al.'s (2008) HyGene model that determines the maximum number of retrieval failures an individual will endure before terminating semantic memory search for plausible interpretations during conditional reasoning. Larger values for Tmax mean that a simulated agent will continue to try to identify potential interpretations when probing their memory for longer, whereas smaller Tmax values mean they will persist in this search for shorter periods of time. Thomas et al. (2008, p. 163) note that this parameter "can be used to model task characteristics, such as time pressure, and individual variables, such as effort or motivation" as it places an upper limit on the amount of cognitive energies a person can/will put towards extracting potential interpretations. Whereas the Tmax parameter corresponds to how long an individual will persist when probing semantic memory during each of the conditional reasoning steps in SiRJ (e.g., Tmax restricts the depth of processing during Steps 1-4), the sjtMax parameter determines the maximum number of times simulated agents will iterate through the entire item evaluation process in SiRJ (e.g., how many times an agent will go through Steps 1-6 in total) before selecting a response if no response options have been identified as the clearly most and least effective choices. Larger values for sjtMax mean that a simulated agent will persist for longer accumulating evidence for/against response options, whereas smaller values mean the agent will persist for shorter periods of time. Tmax and sjtMax are currently represented as independent and exogenous parameters in SiRJ. However, their conceptual similarity suggests these parameters could be correlated and vary endogenously as a function of task characteristics (e.g., deadlines, time pressure) and/or individual differences (e.g., effort, motivation) likely to influence how long an individual will spend attempting to evaluate and respond to an SJT item.

Similarly, the thresholds required to identify a response option as most (MESim) or least effective (LESim) are also currently depicted as exogenous and independent model parameters. Both parameters conceptually represent the amount of evidence an individual requires to make an SJT response. Consequently, there are likely several individual difference (e.g., need for closure, self-regulation preferences, Kruglanski et al., 2000) and task characteristics (e.g., importance/significance of SJT to participant) that could be plausibly related to differences in this parameter across individuals. 
Table B1.

Parameters in SiRJ computational framework

\begin{tabular}{|c|c|c|c|}
\hline Domain & Parameter & Description & $\begin{array}{l}\text { Value used in } \\
\text { Simulations }\end{array}$ \\
\hline \multirow{10}{*}{ Person } & exptLvl & Expertise type of person & Manipulated: $1-5$ \\
\hline & expStrength & Experience strength of person & Manipulated: 1-4 \\
\hline & $L$ & Encoding fidelity of memory traces & .85 \\
\hline & $S$ & $\begin{array}{l}\text { Probability that expertise type of response and situation } \\
\text { context are the same }\end{array}$ & .9 \\
\hline & $A_{c}$ & Threshold for trace activation in episodic memory search & .216 \\
\hline & MESim & $\begin{array}{l}\text { Threshold for cumulative similarity judgment to identify } \\
\text { most effective response }\end{array}$ & .85 \\
\hline & LESim & $\begin{array}{l}\text { Threshold for cumulative similarity judgment to identify } \\
\text { least effective response }\end{array}$ & -.85 \\
\hline & Phi & Size of working memory & 4 \\
\hline & $\operatorname{Tmax}$ & $\begin{array}{l}\text { Maximum number of retrieval failures endured before } \\
\text { terminating semantic memory search }\end{array}$ & 10 \\
\hline & sjtMax & $\begin{array}{l}\text { Maximum number of times person will iterate through item } \\
\text { evaluation process }\end{array}$ & 10 \\
\hline \multirow{5}{*}{ SJT } & numSittlems & Number of SJT items on test & 20 \\
\hline & numSjtRsp & Number of response options per SJT item & 4 \\
\hline & sjtSitSim & $\begin{array}{l}\text { Similarity between specific situation in an SJT item stem } \\
\text { and the prototype of that situation }\end{array}$ & .9 \\
\hline & sjtRspSim & $\begin{array}{l}\text { Similarity between expertise type of responses and the } \\
\text { response options available for SJT items }\end{array}$ & .9 \\
\hline & sitPresent & $\begin{array}{l}\text { Categorical variable indicating whether situational } \\
\text { description is included in SJT item stems }\end{array}$ & $\begin{array}{l}\text { Manipulated: } \\
0=\text { False, } 1=\text { True }\end{array}$ \\
\hline \multirow{12}{*}{ Environment } & MVLength & Number of features in a single minivector & 9 \\
\hline & numSitMv & Number of minivectors in a situation trace & 2 \\
\hline & numSitCxtMv & Number of minivectors in a situation context trace & 2 \\
\hline & numRspMv & Number of minivectors in a response trace & 2 \\
\hline & numRspCxtMv & Number of minivectors in a response context trace & 2 \\
\hline & numSitType & Number of broad situation classes & 3 \\
\hline & numSpecSit & $\begin{array}{l}\text { Number of specific situation types within each broad } \\
\text { situation class }\end{array}$ & 5 \\
\hline & simSitType & $\begin{array}{l}\text { Degree of similarity between broad situation classes } \\
\text { (Simbs) }\end{array}$ & .2 \\
\hline & simSpecSit & $\begin{array}{l}\text { Degree of similarity between specific situation types within } \\
\text { each broad situation class (Simss) }\end{array}$ & .75 \\
\hline & sitTypeProb & $\begin{array}{l}\text { Prevalence/probability of broad situation class } b s \text { in the } \\
\text { environment }\left(P_{b s}\right)\end{array}$ & $\begin{array}{l}\mathrm{P}_{\mathrm{bs} 1}=.8 \\
\mathrm{P}_{\mathrm{bs} 2}=.6 \\
\mathrm{P}_{\mathrm{bs} 3}=.5\end{array}$ \\
\hline & brSitVar & $\begin{array}{l}\text { Scaling factor influencing prevalence/probability of specific } \\
\text { situations within a broad situation class }\end{array}$ & 20 \\
\hline & numExptLvls & Number of qualitatively different expertise types & 5 \\
\hline
\end{tabular}

Note. Names in the Parameter column correspond to the names of the associated variable as they appear in the computational model code (available at https://osf.io/uwdfm/) 
SJT Response Process Theory 84 
Figure B1. Visualization of situation construction used in SiRJ simulations

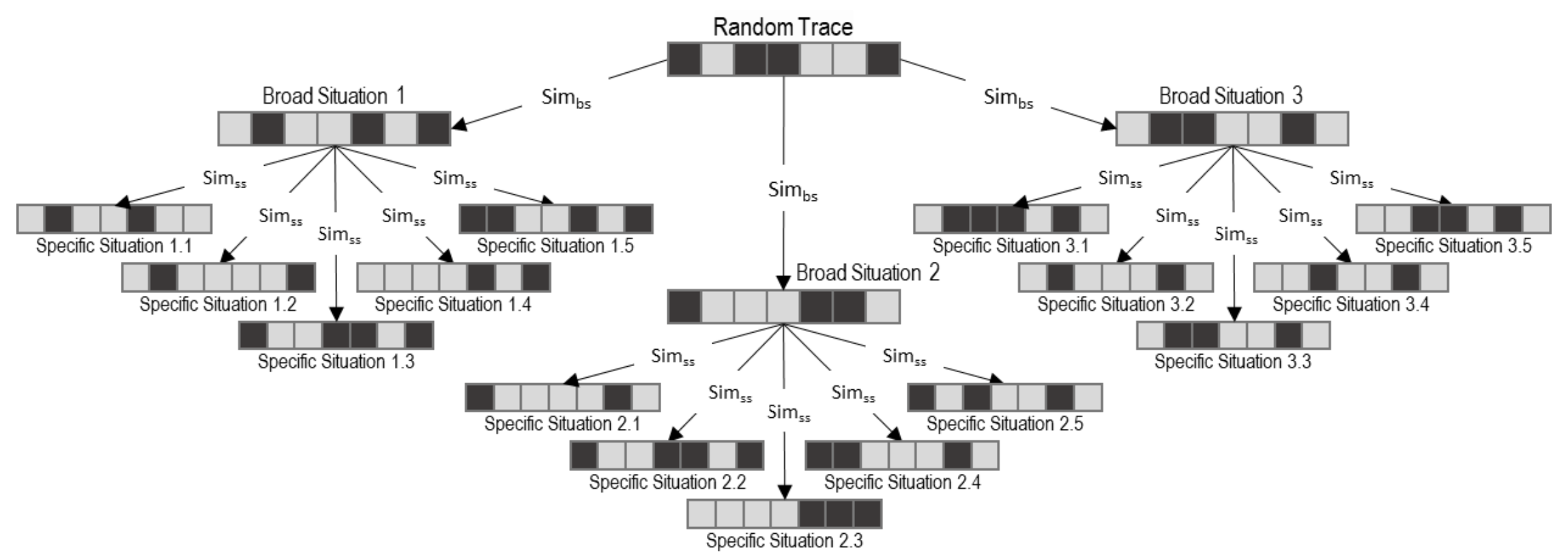

Note. Shaded cells within a trace represent the presence (dark) or absence (light) of a situational feature. Sim bs $_{\text {and }}$ Sim $_{s s}$ correspond to the simSitType and simSitSpec parameters defined in Table B1 and represent the degree of similarity between linked traces. The situation traces displayed here are illustrative; the traces used in the reported simulations contained a total of 18 possible features and thus contained many more possible combinations of features. 


\section{APPENDIX C}

\section{Analysis Description}

The analyses conducted for RQ1-RQ3 used a neural network classification model to examine the extent to which the parameters manipulated in the SiRJ simulations produced reliably different patterns of SJT item response selections by simulated respondents. The analyses conducted for RQ4 used hierarchical Bayesian survival analysis to examine differences in response speed for most versus least effective responding in the simulated and empirical data. Details on the specification of both analytic approaches in the present paper are summarized below.

\section{Neural Network Classification Model}

A multilayer perceptron (MLP) was used to perform the classification analyses on the simulated SJT response selections generated in SiRJ. An MLP is a specific type of feed-forward supervised neural network comprised of at least three node layers; an input layer, output layer, and one or more hidden layers. MLPs utilize a

backpropagation algorithm to iteratively and recursively learn an optimal set of combinatorial weights across nodes in a neural network that can be used to predict which of a set of a priori determined outcome classes best characterizes a set of input variables. The task for the MLP in the present study was to predict a simulated respondent's unobserved expertise type, experience strength, the criterion used to evaluate response preferences, and the type of SJT completed based on the observed response options it selected as most and least effective across all SJT items. In machine learning contexts, this type of problem is described as multi-class multi-label classification because the outcome variables to be predicted are not mutually exclusive (i.e., a respondent has a standing on all four simulation attributes) and each variable contains multiple categorical levels (e.g., Expertise Type 1-5, etc.).

An MLP with a single hidden layer was constructed to carry out the multi-class multi-label classification tasks for RQ1-RQ3; Figure C1 provides a heuristic visualization of the final model. The variables fed into the input layer of the model consisted of a one-hot encoded vector of the response options selected as most and least effective for each SJT item. A simulated test taker's responses to a single SJT item was represented as a string of eight binary variables reflecting the four response options (A-D) that could be selected as most effective and the four response options that could be selected as least effective. For example, the input string for the first SJT item was represented as:

$$
\text { InputsJT_1 }_{\text {I }}\left\{M E 1 \_A, M E 1 \_B, M E 1 \_C, M E 1 \_D, L E 1 \_A, L E 1 \_B, L E 1 \_C, L E 1 \_D\right\}
$$


Using this representation, ME1_A took on the value of 1 if a respondent chose response option $A$ as the most effective response for Item 1 and a value of 0 otherwise. Note that respondents could not select the same response option as both the most and least effective response. Thus, and as exemplified on the left-hand side of Figure C1, the input string for a respondent that selected Response Option B as most effective and Response Option A as least effective for the first SJT item would have been entered into the MLP as:

$$
\text { Input }_{S T_{-} 1}=\{0,1,0,0,1,0,0,0\}
$$

Concatenating these input strings across all 20 SJT items resulted in a single input response vector of length 160 for each respondent.

The target output variables for the MLP were similarly represented as a one-hot encoded vector reflecting the simulated respondent's expertise type, experience strength, the criterion used to evaluate response preferences, and the type of SJT completed. A simulated test taker's standing on these variables was represented by a string of 13 binary variables representing the four levels of experience strength, five levels of expertise, two levels of evaluation criterion, and two levels of SJT type. The output string for a respondent can thus represented as:

Output $=\{$ ExStr1, ExStr2, ExStr3, ExStr4, Expt1, Expt2, Expt3, Expt4, Expt5, RspBeh, RspCons, SitAbs, SitPres $\}$

Each variable in this output string took on a value of 1 if a respondent was assigned that attribute in the simulation and 0 otherwise. As exemplified on the right-hand side of Figure $\mathrm{C} 1$, the output string for a respondent with Experience Strength 2, Expertise Type 1, who used the anticipated consequences of behaviors as the evaluative criterion in SiRJ, and who completed an SJT with item stems present would be represented in the MLP as:

$$
\text { Output }=\{0,1,0,0,1,0,0,0,0,0,1,0,1\}
$$

The hidden layer in the MLP network consisted of a single layer with 80 nodes. There are no widely accepted standards regarding the number of hidden layers nor the number of nodes to include in hidden layers of neural networks (Oswald \& Putka, 2015). However, general rules-of-thumb suggest that a single hidden layer is often sufficient for all but the most complex problems and that the "optimal" number of hidden layer nodes typically lies somewhere between the number of nodes included in the input and output layers. The MLP was examined with both fewer (40) and more (100) nodes in the hidden layer, but no appreciable differences in its predictive capabilities were observed. Hidden layer nodes used the rectified linear unit (ReLU) transfer function to determine node activation and 
combine model input weights. Activation of nodes in the output layer relied on a sigmoid transfer function in order to allow the probabilities of each output class to be computed independently from one another.

A $k$-folds cross-validation strategy without stratified sampling was used to train and validate the MLP with the simulated dataset. This was carried out by first randomly shuffling and then splitting the data into $k$ groups each containing an equal amount of observations. Next, one of the $k$ data subsets was removed and held out as a validation sample. The input and output data from the remaining $k-1$ groups of data were then used to train the neural network and establish its combinatorial weights. The accuracy of this trained model was then evaluated by examining the extent to which the output data from the validation sample was accurately predicted using the input data from the validation sample. This holdout-train-evaluate process was repeated $k$ times, such that each of the unique $k$ data groups was used as the validation sample once and used during model training $k-1$ times. The prediction accuracy and computed weights from each of the $k$ runs was then averaged together to evaluate the model's predictive capacity and the final predictive model (respectively). In the present paper, $k=10$.

The neural network models were built in R using the tensorflow (Allaire \& Tang, 2019) and keras (Allaire \& Chollet, 2019) packages. The tensorflow package in $\mathrm{R}$ provides a high-level interface to the open source software TensorFlow, which provides a computational library for building, training, running, and validating neural networks. Similarly, the keras package in R provides a high-level interface to Keras, an open source API for constructing and compiling neural networks that interfaces with TensorFlow. All R code and data used to run the MLP network as well as the final output reported in the manuscript are available for download at https://osf.io/uwdfm/.

\section{Survival Analysis Regression}

Survival analyses are a set of statistical approaches for exploring the effect of predictors on time until occurrence of an event (Mills, 2010). The event of interest in the present data was response selection for an SJT item and the primary predictor whether that response choice was for the most or least effective option. To examine this relationship, a parametric proportional hazards model using the Weibull distribution to represent time to SJT response selection was used. This model reports the effect of potential covariates on the observed hazard rate (i.e., the conditional probability that an event will occur at a particular time given that it is has not occurred yet) in log-odds. A key advantage of survival analytic techniques is that they can account for observations in which an event did not occur before the observation period concluded in the final model estimates. This type of data is referred to as rightcensored data. Although there were no right-censored observations in the empirical data, there were a number of occasions in the simulated data in which respondents had not chosen a most and/or least effective response 
selection before the maximum number of iterations permitted by the simulation was reached. This data was treated as censored in the survival analyses.

The simulated and real response time data included multiple observations of SJT items nested within individuals. Consequently, two-level (Level $1=$ item, Level 2 = person) hierarchical Bayesian regression was used to account for both person- and item-level effects in both datasets. Survival analysts often refer to the inclusion of random effects to account for heterogeneity in individual rates as frailty models (Mills, 2010). The motivations for using Bayesian estimation were threefold (Kruschke, 2015; Kruschke, Aguinis, \& Joo, 2012; Zyphur \& Oswald, 2015). First, Bayesian methods promote interpretation of distributions (rather than point estimates) for parameters. Second, Bayesian methods rely on the interpretation of credibility intervals (rather than confidence intervals) that provide a sense of the most "believable" values for a modeled parameter given the observed data and prior knowledge/beliefs. Lastly, the results obtained through Bayesian estimation offer a natural route for accumulating evidence through empirical replication by incorporating posterior estimates as prior distributions in future research.

As noted in the manuscript text, the multilevel regression models for the empirical and simulated data controlled for a number of relevant covariates to better estimate the main effect of response type on response speed. Specifically, the regression model fit to the empirical data was the following:

$$
\begin{aligned}
\text { Level 1: } & \mathrm{RT}_{i j}=b_{0 i}+b_{11}\left(\text { RspType }_{i j}\right)+b_{2}\left(\text { WordCnt }_{i j}\right)+b_{3 i}\left(\text { Order }_{i j}\right)+e_{i j} \\
\text { Level 2: } & b_{0 i}=\beta_{00}+r_{i} \\
b_{1 i} & =\beta_{10}+r_{i} \\
b_{2 i} & =\beta_{20}+r_{i} \\
b_{3 i} & =\beta_{30}+r_{i},
\end{aligned}
$$

in which $\mathrm{RT}_{i j}$ was the response time in seconds required to select a response for person $i$ on item $j$, RspType $_{i j}$ was the type of response selection made for the item $\left(0=\right.$ least effective, $1=$ most effective), WordCnt ij $_{\text {Was }}$ the combined number of words (grand-mean centered) in the item stem and response options for an item, and Order $i j$ was the order in which the SJT item was completed $(0=$ first item completed). The regression model fit to the simulated data was similar, but controlled for different covariates:

Level 1: RT $T_{i j}=b_{0 i}+b_{1 i}\left(\right.$ RspType $\left._{i j}\right)+b_{2}\left(\right.$ ItemStem $\left._{i j}\right)+b_{3 i}\left(\right.$ EvalCrt $\left._{j}\right)+e_{i j}$

Level 2: $b_{0 i}=\beta_{00}+r_{i}$

$b_{1 i}=\beta_{10}+r_{i}$

$b_{2 i}=\beta_{20}+r_{i}$

$b_{3 i}=\beta_{30}+r_{i}$ 
in which $\mathrm{RT}_{i j}$ was the response time measured by the number of iterations through the $\mathrm{SiRJ}$ item evaluation process required to make a response for simulated respondent $i$ on item $j$, RspType $e_{i j}$ was the type of response selection made

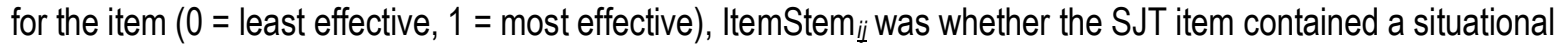
description ( 0 = situation-absent, 1 = situation-present), and EvalCrt $t_{j}$ was the evaluation criterion used by the simulated respondent to determine response preferences $(0=$ similarity of anticipated consequences, $1=$ similarity of behavioral actions). No interactions among variables were included in either model as only the main effect findings were of interest. The correlation among intercepts and slopes in both datasets was modeled.

In order to perform Bayesian parameter estimation, investigators must specify (1) a likelihood distribution that reflects the distribution of observed data and (2) prior distributions that reflect beliefs about plausibility of potential parameter values. The Weibull distribution was used as the likelihood distribution for response speed for both the empirical and simulated datasets. The Weibull distribution is among the most commonly used distributions for response time data and has a long history of use in survival modeling (Mills, 2010). Weakly informative/noncommittal prior distributions were provided over the Level-2 regression coefficients (normal distributions with $M=0, S D=10$ ) in both regression models and permitted the observed data to be the primary contributor to the estimation of the posterior distribution.

All models were carried out in $\mathrm{R}$ using the runjags package (Denwood, 2016). The runjags package provides an interface for performing Markov Chain Monte Carlo estimation routines using the JAGS v4.3.0 software (Plummer, 2013). JAGS is an open source software platform for analyzing Bayesian graphical models using Gibbs sampling techniques. Because the size of the simulated dataset was so large (1.6 million observations across 10,000 respondents), the posterior distributions of the parameter estimates using these data were generated by running the regression model on 50 subsets of the data each comprised of 200 individuals and then combining the parameter estimates calculated from each group into a single combined poster distribution using the parallelMCMCcombine package (Miroshnikov \& Conlon, 2014). 
Figure C1. Visualization of neural network classification model

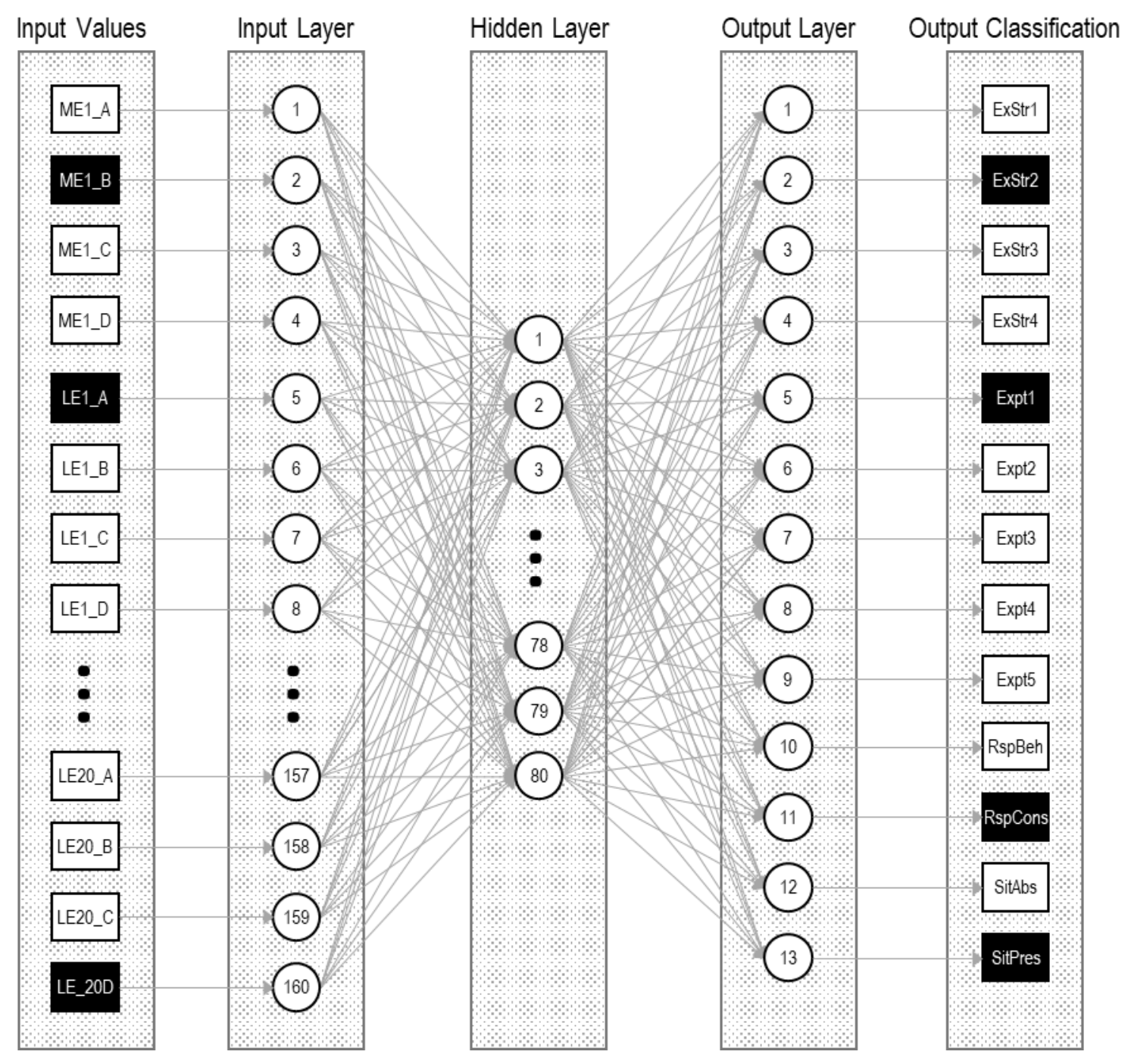

Note. Input values and output classifications are binary $(0=$ false, $1=$ true $)$. Key for input variables: $M E=$ most effective, $L E=$ least effective \# = item number, $A-D=$ response option selected. For example, ME1_B indicates whether the respondent selected response option $B$ as the most effective response for item 1. Key for output variables: ExStr = experience strength, Expt = expertise type, RspBeh = similarity of behavioral actions used as the evaluative criterion, RspCons = similarity of anticipated consequences used as the evaluative criterion, SitPres = situation-present SJT, SitAbs = situation-absent SJT. For example, Expt4 indicates whether the respondent was assigned expertise type 4 in the simulation. The shaded black boxes shown in the input values and output classification columns provide an example of the input and output strings for a single respondent. 


\section{Footnotes}

${ }^{1}$ In support of this general claim, Krumm et al. (2015) report results from a follow-up verbal protocol analysis of individuals completing situation-absent SJT items in which respondents commented that the response options for various items were helpful for accomplishing team goals, signaled particular norms within a team, etc. The fact that respondents explicitly commented on this team context strongly suggest they were interpreting the utility of potential responses with respect to an inferred situational context, even though specific situational descriptions were never provided.

2 HyGene is a direct extension of an earlier computational model of conditional reasoning and human memory (MINERVA-DM; Dougherty, Gettys \& Ogden, 1999). Dougherty et al. (1999) demonstrated how this predecessor of HyGene-and thus HyGene-is also able to account for several commonly reported empirical patterns in the heuristics/cognitive biases literature (e.g., hindsight, availability, overconfidence, etc.)

${ }^{3}$ The representation of different thresholds for delineating a respondent's most and least effective response options is purposeful and reflects that these decisions are distinct choices in the same way that, say, identifying one's favorite food item off a restaurant menu can be performed separately from identifying one's least favorite. The SiRJ response process model posits that individuals appraise the perceived effectiveness for each response option during the item evaluation process (operationalized as the cosine similarity computed during Step 5). The appraisal process for each response option is based on the same underlying cognitive mechanism (conditional reasoning) and criterion (e.g., similarity to the expected outcome of one's likely/preferred response); however, each evaluation is conducted separately and is unaffected by the other options or presence of decision thresholds. Consequently, if one only wished to model a respondent's selection of the most effective but not least effective SJT response option, then only the positive threshold parameter need be specified. In either case, the appraisal and preference accumulation processes would be carried out the same and would produce the same numerical results in the computational model of SiRJ.

${ }^{4} \mathrm{An}$ anonymous reviewer suggested comparing an additional and potentially simpler alternative model in which respondents do not generate and use their own likely/preferred response to a SJT scenario as a point of comparison for evaluating the effectiveness of provided response options, but instead simply compare the desirability of outcomes associated with each response option. This alternative model is functionally equivalent to the theory of cognitive acuity described by Leeds (2012) in which respondents are proposed to detect and compare the "effectiveness signals" of response options for an SJT item when making their preferred selection. Although this 
alternative model seemingly simplifies the proposed item evaluation process by removing steps 2 and 3 and modifying steps 5 and 6 such that respondents only compare the response options to one another, it lacks a mechanism and/or assumptions for describing how the effectiveness of each response option (or their consequences) should be defined. For example, to model Leeds (2012) theory of cognitive acuity, one would need to specify the origin and conceptualization of a response option's perceived effectiveness signal. Where does that evaluation come from? What information is used to determine whether a response's perceived consequences are desirable or effective? Should the perceived effectiveness of a response option be the same across all test takers? If not, what accounts for why it differs? Specifying such core generative mechanisms are critical to the development of computational theories and models (Kozlowski et al., 2013). In SiRJ, the conditional reasoning and similarity judgments described in steps 2, 3, 5, and 6 are the proposed generative mechanisms responsible for how respondents determine the perceived effectiveness of a response option (i.e,. response options with perceived consequences more similar to those of the respondents' likely/preferred response are seen as more effective).

${ }^{5}$ Thomas et al. (2008) present a variety of simulations in which core variables from the original HyGene model that are also represented in SiRJ were manipulated. Many insights into the effects those parameters exert within SiRJ can be gleaned from that original resource. Additionally, the interactive web application at

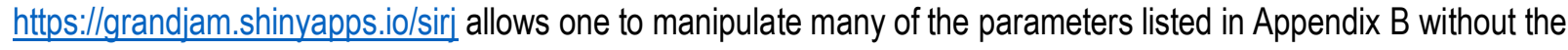
need for computer programming and thus deduce how they impact model initialization and functioning.

${ }^{6}$ The simulation architecture allows for more precise control regarding the construction and representation of dimensions within SJT items if desirable. Given that the present simulations were not attempting to emulate a specific SJT with a particular number of dimensions or items per dimension, a random distribution was sufficient. The random seed used in the reported simulation resulted in 3, 7, and 10 items included on the SJT from each of the three broad situational classes (respectively).

${ }^{7}$ The manuscript reports results in which respondents' selections for the most effective and least effective response options were used as the input response vector to the classification model. Two alternative classification models were also examined in which the input response vector included either respondents' most effective or least effective response selections (but not both). The performance of the classification model making use of both most and least effective response selections tended to be slightly better. This is to be expected given that this model is provided with more diagnostic data points on which to make its predictions. However, the predictive capability of the alternative models that relied only on respondents' most or least effective response selections separately was 
comparable for all predicted parameters and usually within $\sim 10-15 \%$ of the full model's predictive accuracy. Importantly, these results indicate that the parameters manipulated in SiRJ tended to produce unique patterns in both most and least effective response selections and not just one or the other. All data, code, and results from the alternative classification models are available at https://osf.io/uwdfm/.

8 The interpretations for all main effects examined in RQ1-RQ3 are identical for all SJT items not shown. Additionally, the main effect interpretations for RQ1-RQ3 also hold when evaluating the least effective response choices made by simulated respondents. Data and code for examining those data not reported in the figures are available at $\underline{\text { htps://osf.io/uwdfm/. }}$

${ }^{9}$ Associating difficulty with uncertainty, particularly in the context of assessment, may seem ill-advised given that best practices in survey and knowledge/ability test design commonly describe uncertainty as introducing undesired error or noise. However, in the domain of judgment and decision-making, uncertainty has a long history of use in distinguishing decisions characterized as "easy" versus "hard." For example, Kruglanski and Gigerenzer (2011) suggest that a primary challenge for the decision-maker in any judgment task is to (a) identify the possible set of decision rules one can apply to reach a conclusion and (b) select the rule with the highest ecological validity. To the extent that a judgment task is not highly constrained (i.e., is uncertain), the former step is more difficult. Similarly, the latter step is more challenging when the ecological validity of a rule is ambiguous or equivalent to other rules. Similar applications of this logic can be found in Klein's (1997) work on recognition-primed decision making and in Gigerenzer and Hoffrage's (1995) discussions on the effects of information formats on reasoning and judgment. 